
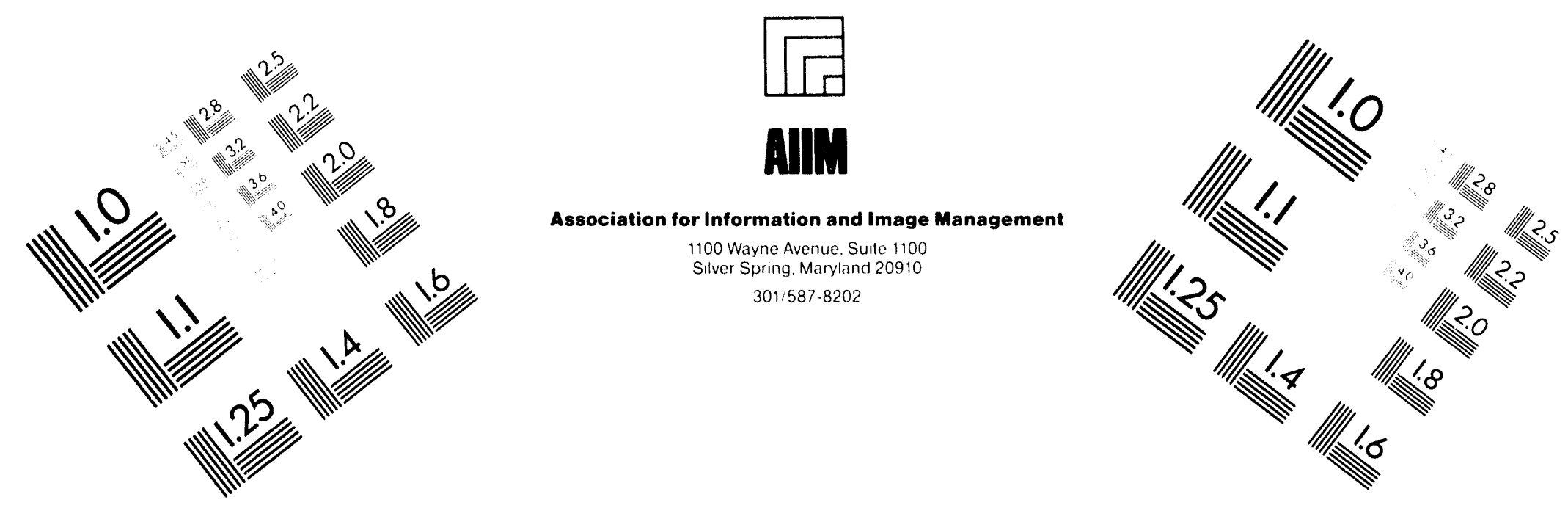

\title{
Centimeter
}

1 Inches
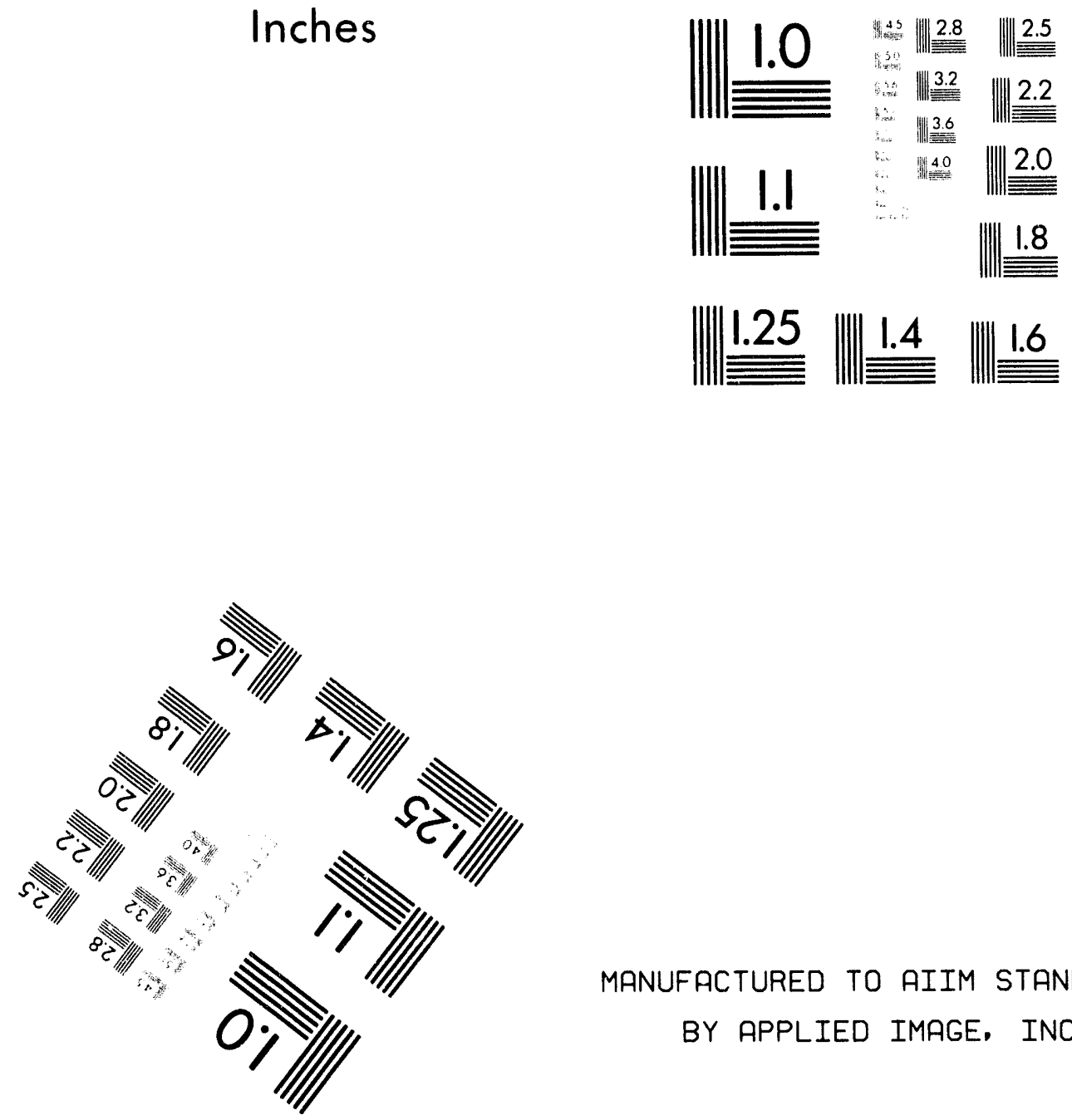

MANUFACTURED TO AIIM STANDARDS

BY APPLIED IMAGE, INC.

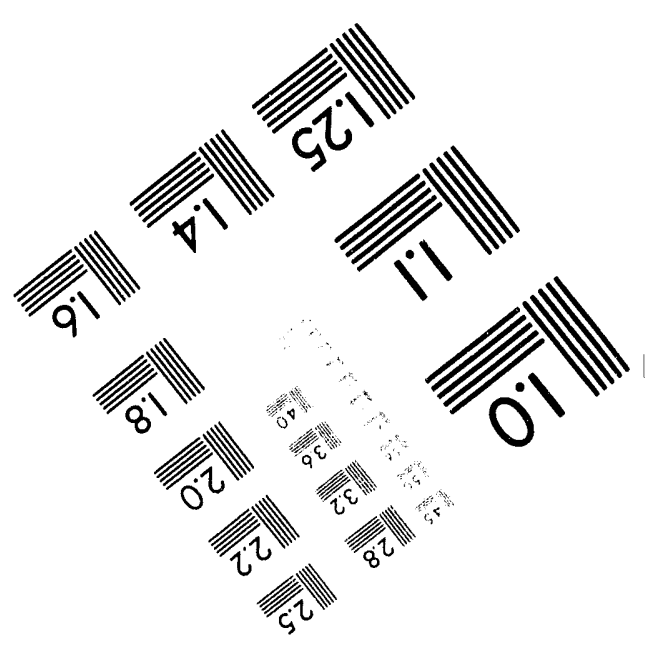



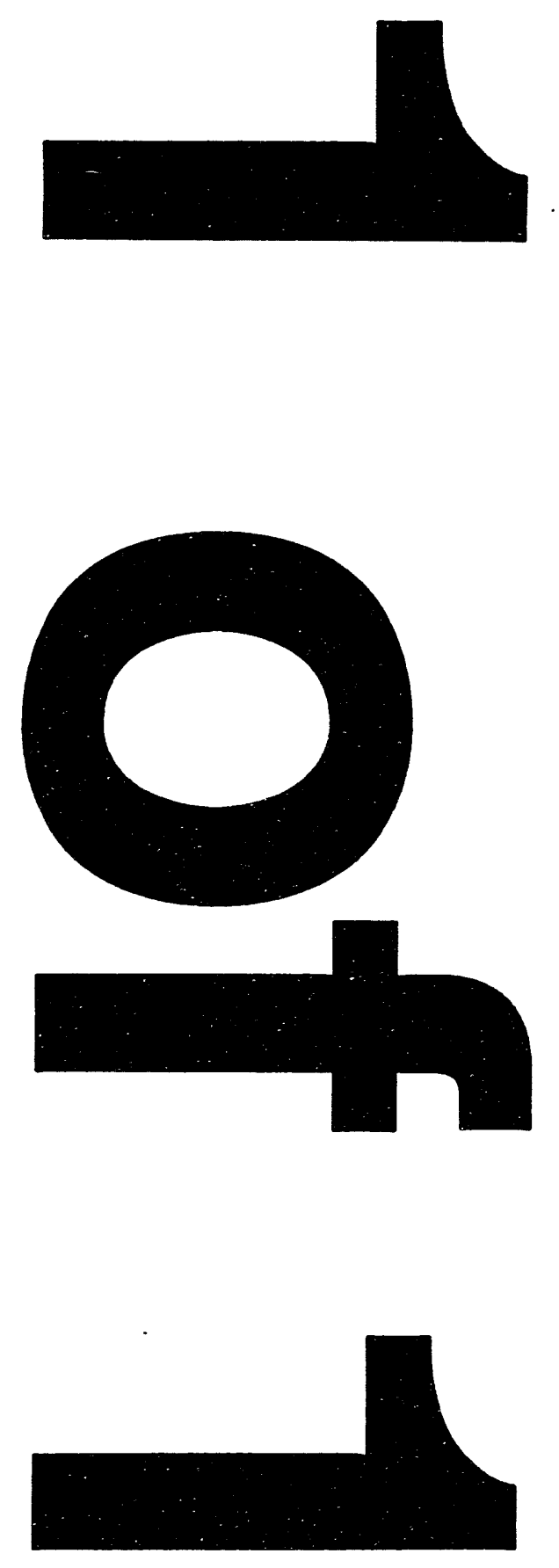


\section{DETECTION AND LOCATION OF MECHANICAL SYSTEM DEGRADATION BY USING DETECTOR SIGNAL NOISE DATA*}

B. Damiano

E. D. Blakeman

L. D. Phillips

Date Published-June 1994

"Research sponsored by the Laboratory Directed Research Program of Oak Ridge National Laboratory.

Prepared by

OAK RIDGE NATIONAL LABORATORY

Oak Ridge, Tennessee 37831-6285

managed by

MARTIN MARIETTA ENERGY SYSTEMS, INC.

for the

U.S. DEPARTMENT OF ENERGY

under contract DE-AC05-84OR21400

by mas 


\section{CONTENTS}

LIST OF FIGURES $\ldots \ldots \ldots \ldots \ldots \ldots \ldots \ldots \ldots \ldots \ldots \ldots \ldots \ldots$

LIST OF TABLES $\ldots \ldots \ldots \ldots \ldots \ldots \ldots \ldots \ldots \ldots \ldots \ldots \ldots \ldots \ldots \ldots \ldots \ldots \ldots$

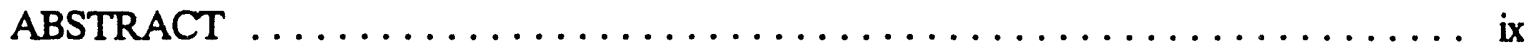

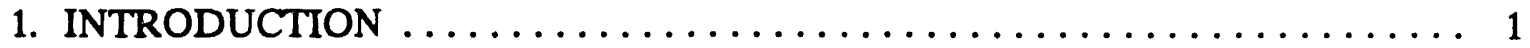

1.1 OVERVIEW OF THE DIAGNOSTIC METHOD ............ 2

1.2 PROGRAM FOR DEVELOPING AND DEMONSTRATING

THE DIAGNOSTIC METHOD $\ldots \ldots \ldots \ldots \ldots \ldots \ldots \ldots \ldots \ldots$

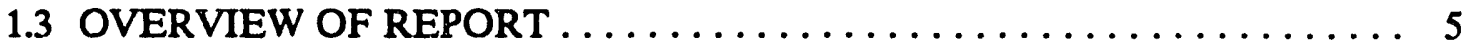

2. PRECURSORY INFORMATION $\ldots \ldots \ldots \ldots \ldots \ldots \ldots \ldots \ldots \ldots \ldots$

2.1 VIBRATION MODEL OF THE MOTOR-PUMP UNIT $\ldots \ldots \ldots \ldots \ldots \ldots$

2.1.1 Mathematical Model Descriptio ................. 6

2.1.2 Calculation of Resonance Frequencies and Mode Shapes . . . . . . . 10

2.1.3 Calculation of the Temporal Response to Initial

Conditions or Applied Forces ................... 10

2.2 APPLICATION OF NEURAL NETWORKS AS CLASSIFIERS/

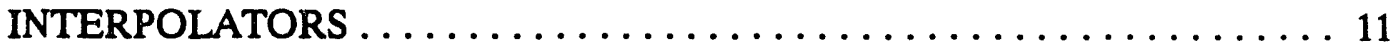

2.2.1 Backpropagation Network .................... 12

3. PHASE I: APPLYING THE DIAGNOSTIC METHOD

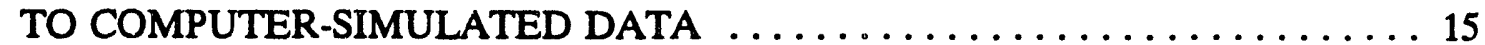

3.1 FORMATION OF THE TRAINING SETS USED DURING PHASE I $\ldots \ldots 15$

3.2 CREATION AND TRAINING OF THE NEURAL NETWORK . . . . . . 18

3.3 RESULTS OBTAINED FROM APPLYING THE DIAGNOSTIC

METHOD TO COMPUTER-SIMULATED DATA . . . . . . . . . . . . . . . 19

3.3.1 Prediction of Spring Rates From Calculated Eigenvalue

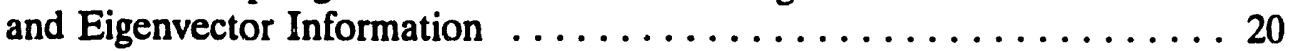

3.3.2 Prediction of Spring Rates from Calculated Vibration Spectra . . . . . . 20

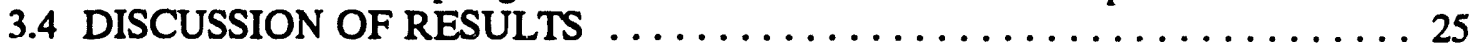

4. PHASE II: APPLYING THE DIAGNOSTIC METHOD TO

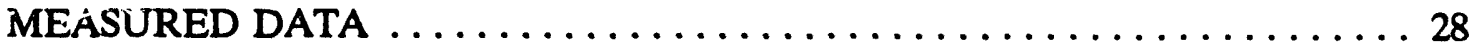

4.1 THE BENCH-TOP TEST UNIT $\ldots \ldots \ldots \ldots \ldots \ldots \ldots \ldots \ldots \ldots \ldots \ldots$

4.1.1 Description of the Bench-Top Test Unit ............... 28

4.1.2 Description of the Data-Acquisition System .............. 31

4.2 MATHEMATICAL MODEL OF THE BENCH-TOP TEST UNIT $\ldots \ldots \ldots 31$

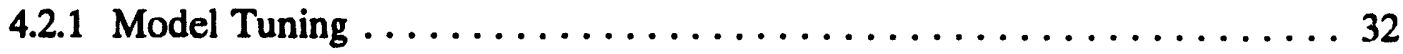

4.2 .2 Measured Data ......................... 33

4.3 FORMATION OF THE TRAINING SETS AND TRAINING OF THE

NEURAL NETWORK $\ldots \ldots \ldots \ldots \ldots \ldots \ldots \ldots \ldots \ldots \ldots \ldots \ldots \ldots$ 
4.4 RESULTS OBTAINED FROM APPLYING THE DIAGNOSTIC

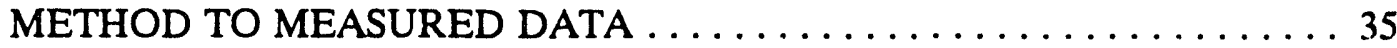

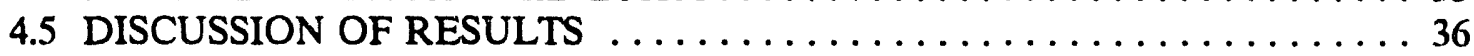

5. SUMMARY AND CONCLUSIONS $\ldots \ldots \ldots \ldots \ldots \ldots \ldots \ldots \ldots \ldots \ldots \ldots$

5.1 RESULTS AND CONCLUSIONS BASED ON THE COMPUTER

SIMULATION RESULTS . . . . . . . . . . . . . . . . . . . . . . . 39

5.2 RESULTS AND CONCLUSIONS BASED ON THE APPLICATION OF THE DIAGNOSTIC METHOD TO THE BENCH-TOP TEST UNIT . . . . 40

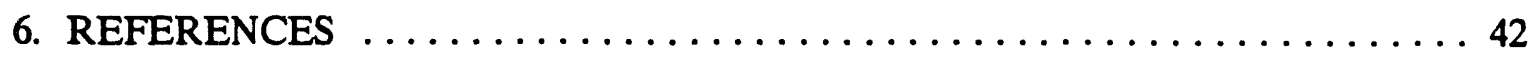

Appendix A. FREQUENCY SPECTRUM DECOMPOSITION TECHNIQUE . . . . 45 Appendix B. PHASE I MODE SHAPES $\ldots \ldots \ldots \ldots \ldots \ldots \ldots \ldots \ldots \ldots$

Appendix C. DETAILED DRAWINGS OF THE BENCH-TOP TEST UNIT . . . 57

Appendix D. MEASURED RESONANCE FREQUENCIES AND MODE-SHAPE COMPONENTS FROM THE

BENCH-TOP TEST UNIT $\ldots \ldots \ldots \ldots \ldots \ldots \ldots \ldots \ldots \ldots$ 


\section{LIST OF FIGURES}

$\underline{\text { Figure }}$

Page

1.1 Block diagram of the method for interpreting detector signal noise data $\ldots \ldots 4$

$2.1 \quad$ Three-beam pump model $\ldots \ldots \ldots \ldots \ldots \ldots \ldots \ldots \ldots \ldots \ldots \ldots$

2.2 Generalized backpropagation neural network with $L$ hidden layers $\ldots \ldots \ldots 13$

2.3 Neural network processing element number $j$ in hidden layer $i \ldots \ldots \ldots$

3.1 Comparison of the known and estimated spring rates for the values

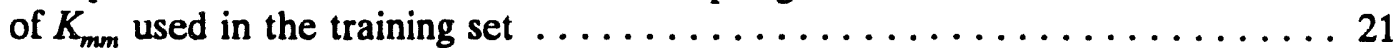

3.2 Comparison of the known and estimated spring rates for the values

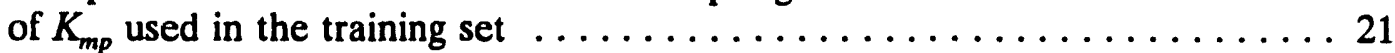

3.3 Comparison of the known and estimated spring rates for the values

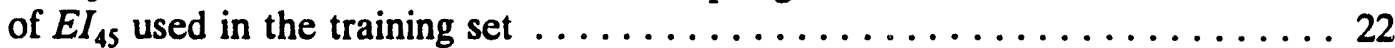

3.4 Comparison of the known and estimated spring rates for the values of $K_{m m}$ between those used in the training set $\ldots \ldots \ldots \ldots 22$

3.5 Comparison of the known and estimated spring rates for the values of $K_{m p}$ between those used in the training set $\ldots \ldots \ldots \ldots \ldots \ldots \ldots \ldots$

3.6 Comparison of the known and estimated spring rates for the values of $E I_{45}$ between those used in the training set $\ldots \ldots \ldots \ldots \ldots \ldots$

3.7 Effect of the number of training set members on neural network estimation accuracy for the 3 training set types used during Phase I . . . . . . 24

4.1 The three-beam model of the bench-top test unit $\ldots \ldots \ldots \ldots \ldots \ldots \ldots$

4.2 Effect of internal pressure on the spring rate of the Firestone 1M1A airspring . 30

4.3 Comparison of the measured and calculated values of resonant frequency $\ldots 34$

4.4 A comparison of the known and estimated spring rate values for spring rates

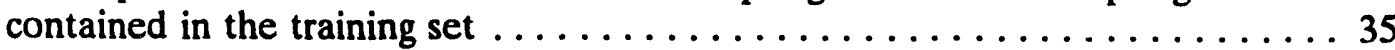

4.5 A comparison of the known and estimated spring rate values made

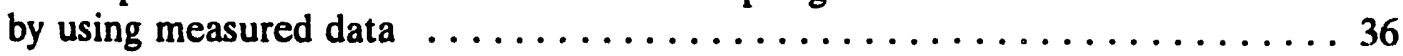

4.6 Estimated value of $K_{m m}$ calculated while holding the value of $K_{m p}$ fixed . . . . 37 
4.7 Estimated value of $K_{m p}$ calculated while holding the value of $K_{m m}$ fixed . . . 37

B.1 Mode shapes A through F calculated by using $K_{m m}=1 \times 10^{5} \mathrm{lb} /$ in.

$\left(1.75 \times 10^{7} \mathrm{~N} / \mathrm{m}\right), K_{m p}=1 \times 10^{5} \mathrm{lb} /$ in. $\left(1.75 \times 10^{7} \mathrm{~N} / \mathrm{m}\right)$,

$E I_{45}=1 \times 10^{6} \mathrm{lb}_{\mathrm{f}} \cdot \mathrm{in}^{2}\left(2.87 \times 10^{3} \mathrm{~N} \cdot \mathrm{m}^{2}\right)$

B.2 Mode shapes $\mathrm{G}$ through $\mathrm{K}$ calculated by using $K_{m m}=1 \times 10^{5} \mathrm{lb}_{\mathrm{f}}$ in.

$\left(1.75 \times 10^{7} \mathrm{~N} / \mathrm{m}\right), K_{m p}=1 \times 10^{5} \mathrm{lb} /$ in. $\left(1.75 \times 10^{7} \mathrm{~N} / \mathrm{m}\right)$,

$E I_{45}=1 \times 10^{6} \mathrm{Ib}_{\mathrm{f}} \cdot \mathrm{in}^{2}\left(2.87 \times 10^{3} \mathrm{~N} \cdot \mathrm{m}^{2}\right)$

B.3 Mode shapes A through F calculated by using $K_{m m}=5 \times 10^{5} \mathrm{lb} / \mathrm{in}$.

$\left(8.76 \times 10^{7} \mathrm{~N} / \mathrm{m}\right), K_{m p}=5 \times 10^{5} \mathrm{lb} /$ in. $\left(8.76 \times 10^{7} \mathrm{~N} / \mathrm{m}\right)$,

$E I_{45}=5 \times 10^{6} \mathrm{lb}_{\mathrm{f}} \cdot \mathrm{in}^{2}\left(1.44 \times 10^{4} \mathrm{~N} \cdot \mathrm{m}^{2}\right)$

B.4 Mode shapes $\mathrm{G}$ through $\mathrm{K}$ calculated by using $K_{m m}=5 \times 10^{5} \mathrm{lb}_{\mathrm{f}}$ /in.

$\left(8.76 \times 10^{7} \mathrm{~N} / \mathrm{m}\right), K_{m p}=5 \times 10^{5} \mathrm{lb} /$ in. $\left(8.76 \times 10^{7} \mathrm{~N} / \mathrm{m}\right)$,

$E I_{45}=5 \times 10^{6} \mathrm{lb}_{\mathrm{f}} \cdot \mathrm{in}^{2}\left(1.44 \times 10^{4} \mathrm{~N} \cdot \mathrm{m}^{2}\right)$

B.5 Mode shapes $A$ through $F$ calculated by using $K_{m m}=1 \times 10^{6} \mathrm{lb}_{\mathrm{f}} /$ in.

$\left(1.75 \times 10^{8} \mathrm{~N} / \mathrm{m}\right), K_{m p}=1 \times 10^{6} \mathrm{lb} /$ in. $\left(1.75 \times 10^{8} \mathrm{~N} / \mathrm{m}\right)$,

$E I_{45}=1 \times 10^{7} \mathrm{lb}_{\mathrm{f}} \cdot \mathrm{in}^{2}\left(2.87 \times 10^{4} \mathrm{~N} \cdot \mathrm{m}^{2}\right)$

B.6 Mode shapes $\mathrm{G}$ through $\mathrm{K}$ calculated by using $K_{m m}=1 \times 10^{6} \mathrm{lb}_{\mathrm{f}} / \mathrm{in}$.

$\left(1.75 \times 10^{8} \mathrm{~N} / \mathrm{m}\right), K_{m p}=1 \times 10^{6} \mathrm{lb} /$ in. $\left(1.75 \times 10^{8} \mathrm{~N} / \mathrm{m}\right)$,

$E I_{45}=1 \times 10^{7} \mathrm{lb}_{\mathrm{f}} \cdot \mathrm{in}^{2}\left(2.87 \times 10^{4} \mathrm{~N} \cdot \mathrm{m}^{2}\right)$ 


\section{LIST OF TABLES}

Table

Page

$2.1 \quad$ Pump model lumped parameters $\ldots \ldots \ldots \ldots \ldots \ldots \ldots \ldots \ldots \ldots$

3.1 Spring rate values used in the Phase $I$ training sets $\ldots \ldots \ldots \ldots \ldots \ldots \ldots$

3.2 Training sets used in the Phase I portion of the investigation $\ldots \ldots \ldots \ldots 18$

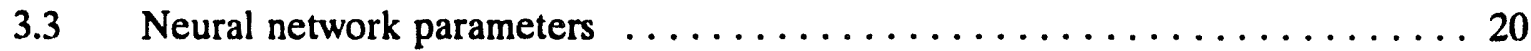

3.4 Comparison of known spring rates with spring rates estimated

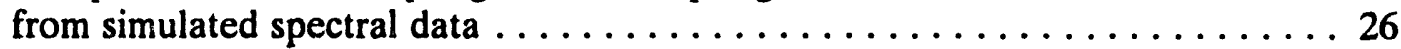

4.1 Bench-top test unit model lumped parameters $\ldots \ldots \ldots \ldots \ldots \ldots \ldots \ldots$

4.2 Resonant frequencies of the bench-top test unit model $\ldots \ldots \ldots \ldots \ldots \ldots 33$ 


\begin{abstract}
This report describes the investigation of a diagnostic method for detecting and locating the source of structural degradation in mechanical systems. The goal of this investigation was to determine whether the diagnostic method would be practically and successfully applied to detect and locate structural changes in a mechanical system. The diagnostic method uses a mathematical model of the mechanical system to define relationships between system parameters, such as spring rates and damping rates, and measurable spectral features, such as natural frequencies and mode shapes. These model-defined relationships are incorporated into a neural network, which is used to relate measured spectral features to system parameters. The diagnosis of the system's condition is performed by presenting the neural network with measured spectral features and comparing the system parameters estimated by the neural network to previously estimated values. Changes in the estimated system parameters indicate the location and severity of degradation in the mechanical system.

The investigation involved applying the method by using computer-simulated data and data collected from a bench-top mechanical system. The effects of neural network training set size and composition on the accuracy of the model parameter estimates were investigated by using computer-simulated data. The measured data were used to demonstrate that the method can be applied to estimate the parameters of a "real" mechanical system.

The results show that this diagnostic method can be applied to successfully locate and estimate the magnitude of structural changes in a mechanical system. The average error in the estimated spring rate values of the bench-top mechanical system was approximately 5 to $10 \%$. This degree of accuracy is sufficient to permit the use of this method for detecting and locating structural degradation in mechanical systems. It is also shown that the neural network training sets required for this level of estimate accuracy are not impractically large and can consist of natural frequency and mode shape information that is sufficient to provide system parameter estimates.
\end{abstract}




\section{INTRODUCTION}

This report describes the investigation of a new diagnostic method for detecting and locating the source of structural degradation in mechanical systems. The goal of this investigation was to determine whether the diagnostic method would be practically and successfully applied to detect and locate structural changes in a mechanical system. The diagnostic method uses a mathematical model of the mechanical system to define relationships between system parameters such as spring rates and damping rates and to measurable spectral features such as the characteristics of resonance peaks. These model-defined relationships are incorporated into a neural network that is used to relate measured spectral features to system parameters. The diagnosis of the system's condition is performed by presenting the neural network with measured spectral features and comparing the system parameters estimated by the neural network to previously estimated values. Changes in the estimated system parameters indicate the location and severity of degradation in the mechanical system.

This method has a significant advantage over current monitoring methods (which track specific spectral characteristics such as resonance frequencies or the total energy in a frequency interval). Current monitoring methods can detect mechanical degradation but provide little indication of the magnitude or location of the degradation. The main advantage of this new method is that the signature interpretation is based on mathematical model results, allowing a direct association between spectral changes and structural degradation. This approach removes much of the subjectiveness commonly associated with signature interpretation.

Spectral analysis of detector signal noise data is a proven technique for monitoring the condition of mechanical systems. ${ }^{1}$ This technique is well established for monitoring rotating machinery and, more recently, has been applied to monitor the condition of nuclear reactor internal components. ${ }^{1-4}$ The main activities comprising a monitoring program involve data collection and storage, data analysis, and interpretation of analysis results. Of these activities, interpretation remains the least developed, mainly because of the difficulty in automating the intuitive processes involved in interpreting spectral data.

Interpretation of spectral data is typically based on the characteristics of resonance peaks. Resonance peak characteristics are frequency, width, amplitude, and skewness (a measure of asymmetry). A crucial step in interpreting spectra is identifying the cause of each resonance peak. Resonance peaks are normally caused by either internally generated or externally imposed driving forces or by an amplified system response to external driving forces caused by a structural resonance. The source of most peaks caused by internal or external driving forces can usually be identified on the basis of known operating conditions such as rotation speed or can be derived from knowledge or measurement of the external forces. Peaks due to structural resonances can often be identified on the basis of results obtained from measurements made during special tests such as impact testing or coastdown measurements. Additional measured information such as the relative phases between detector signals may be available and can be useful in determining the mode shape corresponding to some structural resonances. When structural degradation occurs, the features of the resonance peaks corresponding to structural resonances change. Thus, changes in resonance peaks indicate that mechanical components have experienced degradation. 
Mathematical simulations of mechanical systems have been used to determine the cause of changes in peaks corresponding to structural resonances. ${ }^{5-8}$ This approach involves development of a mathematical model describing the dynamic behavior of the mechanical system and adjusting the model parameters to obtain agreement between the calculated and measured spectral features. If the measured spectral features change, the model parameters are readjusted to give agreement between the calculated spectral features and the new measurements. The model parameter adjustments indicate where degradation may have occurred in the mechanical system.

The main shortcoming of previous applications of mathematical modeling techniques to interpret spectral data is the lack of an easily implemented, systematic method to adjust the model parameters to obtain agreement between calculated and measured spectral features. This tedious process has been performed manually, involving considerable time and effort and also requiring significant expertise on the part of those adjusting the model parameters. The method investigated by this study uses a neural network to adjust the model parameters, resulting in a systematic and, from the perspective of the user, relritively simple process for matching model parameters to measured spectral features.

\subsection{OVERVIEW OF THE DIAGNOSTIC METHOD}

The diagnostic method combines a mathematical model of the monitored system to relate system parameters to measurable spectral phenomena, a technique to extract the significant features from the frequency spectra, and a neural network to match the extracted spectral features to corresponding system parameters. Five steps comprise the technique.

1. Develop a mathematical model describing the vibrations and dynamics of the monitored mechanical system.

2. Design a neural network that will be trained to simulate the model.

3. Use the mathematical model to form a training set for the neural network. The training set will consist of calculated model responses (i.e., spectral features) as known inputs and the corresponding spring and damping constants (i.e., system or model parameters) as known outputs. Thus, if the relationship between the spectral features and the system parameters is single valued, the neural network will perform the mathematical inverse of the model.

4. Train the neural network by iteratively adjusting the neural network connection weights to obtain optimum agreement between the neural network and the mathematical model.

5. Use the trained neural network to estimate the system parameters corresponding to a measured set of spectral features.

Only the most general discussion about model development will be made here because a unique model must be developed for each application. The modeling technique is independent of the monitoring method. Thus, for some applications, relatively coarse 
lumped-parameter approximations may be suitable; for others, detailed models employing sophisticated modeling techniques such as finite element methods may be needed. The only requirement placed on the mathematical model by the monitoring method is that the model must simulate the significant and measurable effects caused by the changing of system parameters. The parameter most likely to change in mechanical systems is stiffness or damping, and the significant and measurable effects of either of these changes will be the characteristics of the resonance frequencies and mode shapes.

The supervised learning mode of neural network training used in this investigation involves adjusting internal neural network parameters until satisfactory agreement is obtained between the sets of known input and output parameters in the training set. For the neural network to relate spectral features to system parameters, the training set will use spectral features calculated by the mathematical model as neural netw ork input and will use the corresponding model input parameters as neural network output. After training, the neural network will effectively contain all of the significant information available from the model and will, in effect, perform the mathematical inverse of the model.

The implementation of the trained neural network for interpreting vibration signatures is summarized in Fig. 1.1. Sensor signals are conditioned and then transformed into frequency spectra by an fast Fourier transform (FFT) algorithm. The spectral data are decomposed, yielding frequency peaks and mode shape components. These spectral features are used as input to the neural network. The neural network outputs are estimates of the original system parameters. Comparison of the latest estimated system parameters with previously estimated values indicates whether degradation has occurred and the severity of any degradation. The specific mode! parameter that experiences a change indicates the location of the degradation because each model parameter represents a specific system component.

\subsection{PROGRAM FOR DEVELOPING AND DEMONSTRATING THE DIAGNOSTIC METHOD}

The program for investigating the monitoring method consisted of two phases: Phase I involved software tool development and concept demonstration by applying the diagnostic method to computer-simulated data. Phase II applied the software tools developed during Phase I to measured data acquired from a bench-top test unit.

\section{Phase I}

The Phase I activities concentrated on developing software tools and on applying these tools to computer-simulated data. An outline of the steps comprising Phase I is given below:

I.1. Complete the development of the mathematical model of a motor-driven centrifugal pump that had been used in a previous Oak Ridge National Laboratory (ORNL) project. This model was modified to include calculation of resonance frequencies, $Q$ values, and mode shapes. The code output is either the time response of each mass point (when calculating the response to forcing functions) or the frequency, $Q$ value, and mode shape of each natural mode. 


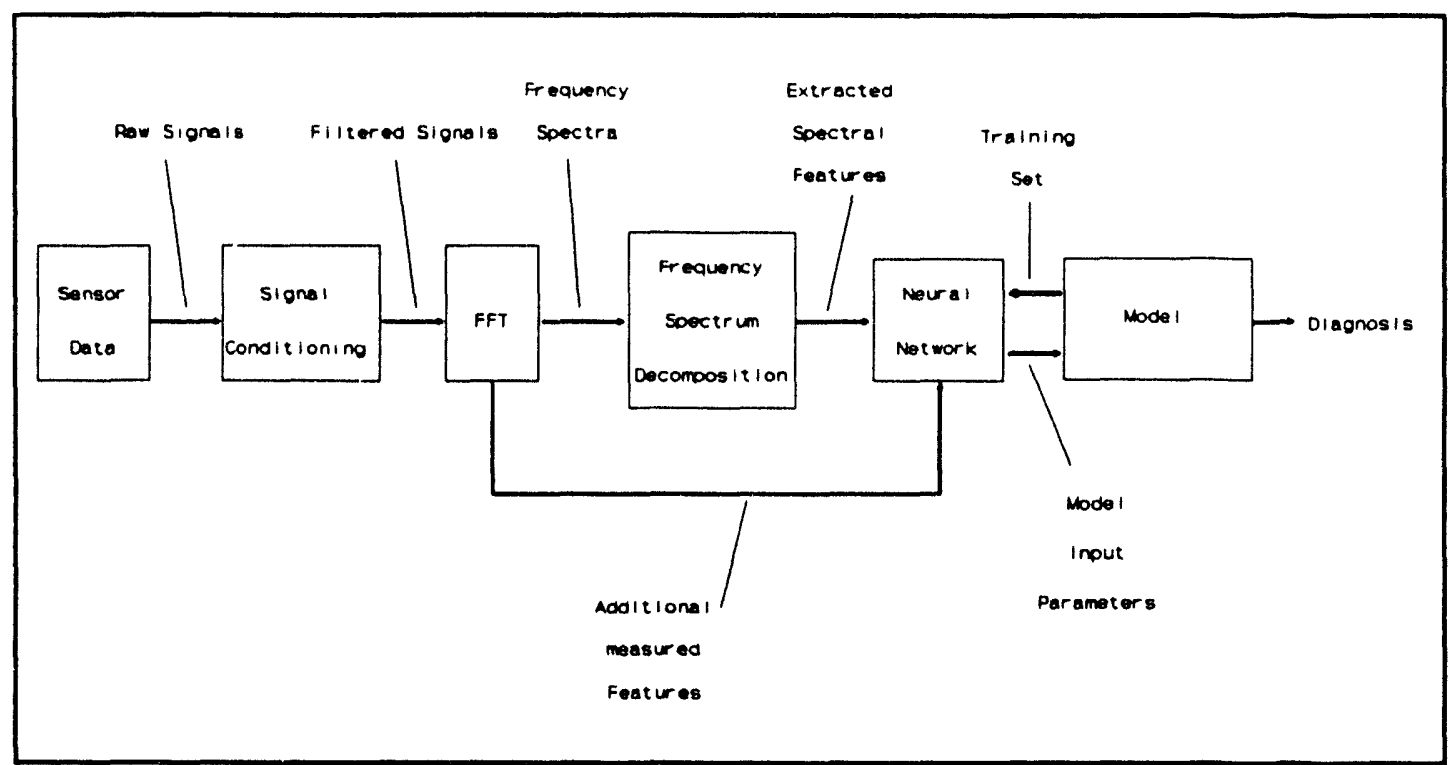

Fig. 1.1. Block diagram of the method for interpreting detector signal noise data $(\mathrm{FFT}=$ fast Fourier transform).

I.2. Form a training set for the neural network. A training set will be formed by using the model developed in Step I.1.

I.3. Create and train the neural network.

I.4. Test the ability of the neural network to reproduce model results by providing the neural network with spectral features calculated by using known model parameters and then comparing these values to those estimated by the trained neural network.

1.5. Test the diagnostic method by using computer-simulated data. This test was done by calculating the response of the model to an initial mass-point deflection for known model parameters, transforming the response into the frequency domain, decomposing the resulting frequency spectrum to obtain the appropriate spectral features, and then providing these to the neural network to estimate the model parameters. Comparison of the known and estimated model parameters was used to evaluate the effectiveness of the diagnostic method.

\section{Phase II}

The Phase II activities concentrate on the creation and instrumentation of the bench-top test unit and the application of the diagnostic method to measured data. An outline of the steps comprising Phase II are given below:

II.1. Select and purchase the equipment required to construct and instrument the bench-top test unit. The bench-top test unit system was constructed so the 
mathematical model developed in Phase I could be easily adapted to model the behavior of the bench-top unit.

II.2. Construct, instrument, and test the bench-top unit.

II.3. Write, test, and debug a code to interface the output from the data acquisition and spectral analysis code to the frequency spectrum decomposition code.

II.4. Form an input data set describing the bench-top unit for the mathematical model developed in Step I.1.

II.5. Form a training set by using the input data set describing the bench-top unit. Train the neural network.

II.6. Form a frequency spectrum, decompose the spectrum, and apply the results of the frequency spectrum decomposition to the neural network by using data collected from the bench-top unit. Introduce stiffness or damping changes to the bench-top system, and observe the ability of the system to detect the effect of the changes and diagnose the location and magnitude of the change. Step II.6 will be used to evaluate the overall feasibility of the diagnostic method.

\subsection{OVERVIEW OF REPORT}

For completeness, Chapter 2 of this report presents background information on mechanical modeling, resonant frequencies, and neural networks for readers unfamiliar with these subject areas. Chapter 3 describes the analytical portion of the Phase I investigation. Chapter 3 includes descriptions of the development of the software tools and the creation and training of the neural network and also presents and discusses the results obtained from applying the diagnostic method to computer-simulated data. Chapter 4 describes the experimental portion of the Phase II investigation. Chapter 4 includes descriptions of the bench-top test unit and its instrumentation, the mathematical model of the bench-top test unit, and the creation and training of the neural network and also presents and discusses the results obtained from applying the diagnostic method to measured data. Chapter 5 summarizes the results of the investigation and draws conclusions concerning the feasibility of the diagnostic method on the basis of those results. 


\section{PRECURSORY INFORMATION}

This chapter presents general background information in the fields of mechanical modeling, resonance frequency and mode shape calculation, and neural networks. Although this information is not required to fully describe the investigation of the monitoring technique, it may be needed to obtain a thorough understanding of the investigation. The subjects discussed are the mathematical model of the mechanical system used in this investigation, a description of the frequency spectrum decomposition technique, and the application of neural networks as classifiers or interpolators.

\section{VIBRATION MODEL OF THE MOTOR-PUMP UNIT}

This section describes the mathematical model of the motor-pump unit used in this investigation. This model is used to calculate resonance frequencies and mode shapes and can also be used to calculate the system response to initial conditions or applied forces.

\subsubsection{Mathematical Model Description}

A lumped parameter model consisting of three interconnected beams, modeled as a series of mass points connected by massless beam sections, represents the motor-driven centrifugal pump (Fig. 2.1). The first beam represents the motor shaft and its attached mass, the second beam represents the pump shaft and its attached mass, and the third beam represents the machine base plate. Different geometry and material properties may be used for each beam section. The motor and pump shafts are connected by a coupling, which is represented by a linear spring and a trunnion spring. The motor and pump shafts are connected to the base plate (i.e., to their casings) by the bearings, which are represented by linear springs and proportional dampers. Two linear spring/proportional damper pairs are used to represent the flexible mounts. The attachment of the flexible mounts to the bench is assumed to be fixed.

The number and the location of the mass points were selected to represent the system's mass distribution and deflection and to allow for the application of external forces. The motor and pump shafts contain the majority of the mass points because these beams model relatively flexible components that experience significant bending and are subjected to a variety of external forces. The steel base plate is approximated as a rigid body because it is stiff relative to the motor shaft, pump shaft, and flexible mounts. Two mass points are used to represent the base-plate mass.

The components represented by each mass point are listed in Table 2.1. Concentrated masses exist at both ends of the motor shaft, at the coupling end of the pump shaft, and between each bearing pair. These mass-point locations allow the application of forces generated by rotating imbalances, coupling misalignments, and flow-induced forces on the impeller. Mass points at the bearing locations allow forces due to bearing defects to be applied to the motor and pump shafts. Portions of the motor or pump shaft mass are included in each of these mass points. The base-plate mass points include the base-plate mass and the mass of the motor and fump cases. The motor and pump cases are assumed to be rigidly attached to the base plate because the stiffness of 


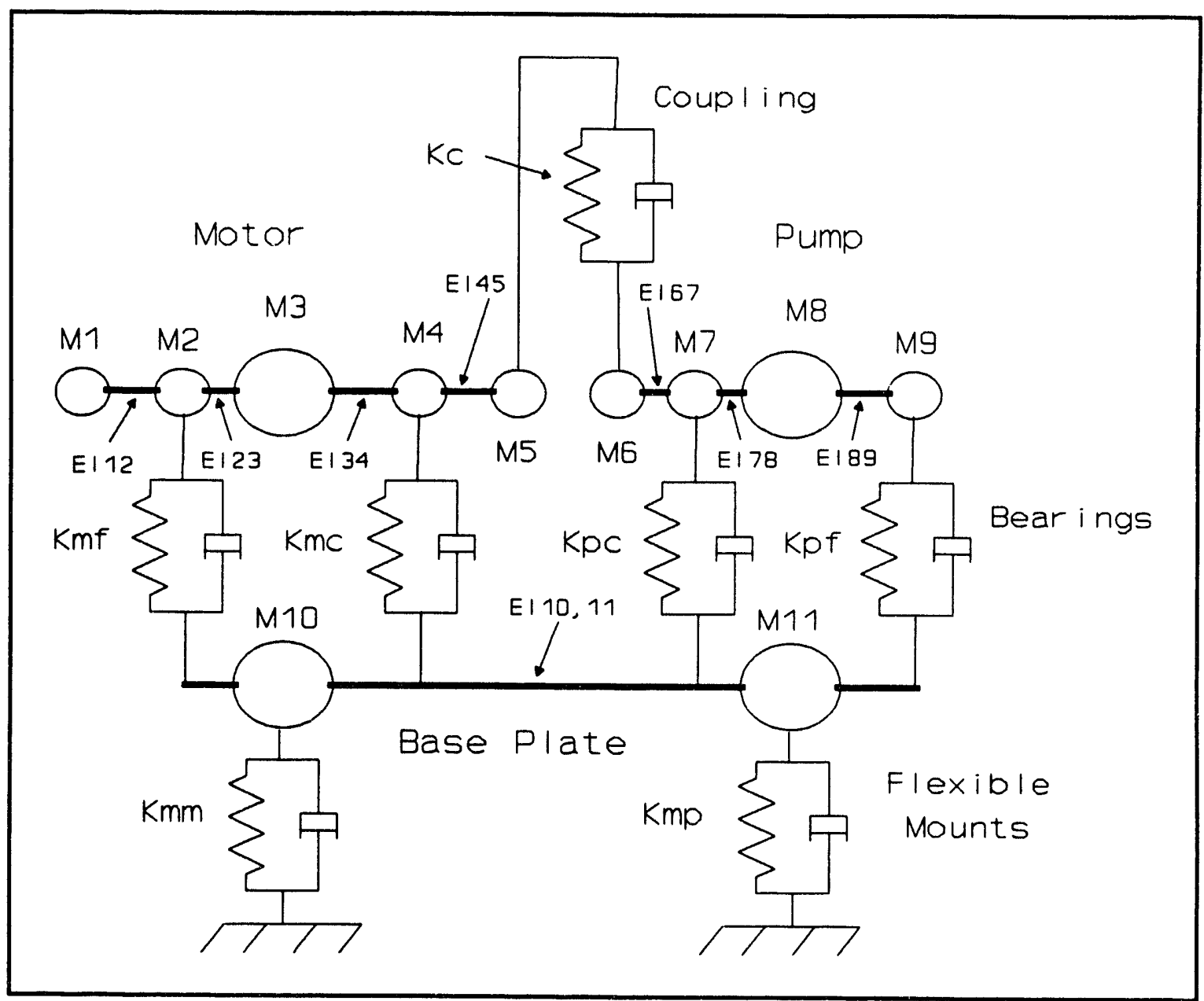

Fig. 2.1. Three-beam pump model (definitions are given in Table 2.1).

the bolted connections joining the motor and pump to the base plate is much greater than the beam stiffness or mounting-spring rates.

The matrix equation of motion for the model is given by

where

$$
M \hat{X}(t)+C \dot{X}(t)+\mathrm{KX}(t)=F(t),
$$

$M=$ the $11 \times 11$ mass matrix,

$\mathbf{C}=$ the $11 \times 11$ damping matrix,

$\mathrm{K}=$ the $11 \times 11$ stiffness matrix,

$F(t)=$ the time-dependent applied force vector,

$X(t)=$ the time-dependent displacement vector,

$\dot{X}(t)=$ the time-dependent velocity vector,

$\ddot{X}(t)=$ the time-dependent acceleration vector. 
Table 21. Pump nodel lumped parameters

\begin{tabular}{cl}
\hline Model parameter & \multicolumn{1}{c}{ System component } \\
\hline$M_{1}$ & Motor fan and part of motor shaft \\
$M_{2}$ & Outboard motor bearing mass, part of motor shaft \\
$M_{3}$ & Motor armature mass, part of motor shaft \\
$M_{4}$ & Inboard motor bearing mass, part of motor shaft \\
$M_{5}$ & Motor half of coupling, part of motor shaft mass \\
$M_{6}$ & Pump half of coupling, part of pump shaft \\
$M_{7}$ & Inboard pump bearing mass, part of pump shaft \\
$M_{8}$ & Pump impeller mass, part of pump shaft \\
$M_{9}$ & Outboard pump bearing mass, part of pump shaft \\
$M_{10}$ & Mass of motor stator, motor case, and part of base plate \\
$M_{11}$ & Mass of pump case and part of base plate \\
$\mathrm{K}_{\mathrm{mb}}, \mathrm{K}_{\mathrm{mc}}$ & Motor bearing spring rate \\
$\mathrm{K}_{\mathrm{pcc}}, \mathrm{K}_{\mathrm{pt}}$ & Pump bearing spring rate \\
$\mathrm{K}_{\mathrm{mm}}, \mathrm{K}_{\mathrm{mp}}$ & Spring rates of the base-plate mounts \\
$\mathrm{EI}_{\mathrm{iv}}$ & Beam stiffness between mass points $i$ and $j$ \\
$\mathrm{~K}_{\mathrm{c}}$ & Coupling spring rate \\
\hline &
\end{tabular}

The mass matrix is a diagonal matrix containing the mass values of the eleven mass points. The elements of the stiffness matrix are calculated by using the method of influence coefficients. ${ }^{9}$ The relationship between the mass-point forces and the deflections can be represented by the matrix equation,

where

$$
d=I C f,
$$

$d=$ the deflection vector,

IC $=$ the influence coefficient matrix,

$f=$ the applied force vector.

An element of the influence coefficient matrix $\mathbf{I C}_{i, j}$, is defined as the deflection at mass point $i$ caused by a unit force applied to mass point $j$. Thus, the elements of the $j$ th column of the influence coefficient matrix are the mass-point location deflections caused by a unit force applied at the location of mass point $j$. The deflections of the model due to forces applied perpendicular to the beam axis at the mass-point locations are calculated by determining the beam reactions, using the reactions and applied forces to form the 
moment equations, analytically integrating the moment equations twice to obtain beam deflection equations, and finally applying boundary and matching conditions to determine the integration constants. Repeating this calculation for unit forces applied at each mass-point location forms the columns of the influence coefficient matrix. Equation (2.2) can be solved for the applied force vector by premultiplying by the inverse of the influence coefficient matrix,

$$
f=\mathrm{IC}^{-1} d=\mathrm{Kd}
$$

where

$\mathrm{IC}^{-1}=\mathbf{K}$ is the stiffness matrix.

After the mass and stiffness matrices are formed, the undamped equation of motion,

$$
\mathbf{M} \tilde{X}(t)+\mathbf{K X}(t)=F(t),
$$

can be used to find the undamped natural frequencies (eigenvalues) and mode shapes (eigenvectors). A coordinate transformation that uncouples the equations can be formed by using the modal matrix $\mathbf{P}$, whose columns are the eigenvectors of Eq. (2.4). The modal matrix and its transpose are orthogonal with respect to the mass and stiffness matrices because of the orthogonality of the eigenvectors. Using the modal matrix to perform a coordinate transformation results in uncoupling the equations of motion,

$$
P^{T} M P=M_{n}=\left[8_{i, j} m_{i}\right](i, j=1 \text { to } 11),
$$

and

$$
\mathrm{P}^{\mathrm{T}} \mathrm{KP}=\mathrm{K}_{n}=\delta_{i, j} k_{i}(i, j=1 \text { to } 11),
$$

where $\mathrm{M}_{n}$ and $\mathrm{K}_{n}$ are the mass and stiffness matrices in principal coordinates, $m_{i}$ and $k_{i}$ are the modal mass and modal stiffness of the $i$ th mode, and $\delta_{i, j}$ is the Dirac delta function.

If the modal damping ratio $\xi_{i}$ is known for each mode, the elements in the damping matrix in principal coordinates $\mathbf{C}_{\mathbf{n}}$ (which is diagonal if proportional damping is assumed) can be determined by using

$$
C_{m, i}=2 \xi_{i} m_{i}^{1 / 2} k_{i}^{1 / 2}=2 \xi_{i} m_{i} \omega_{n, i}
$$

where $\omega_{n, i}=$ the eigenvalue of mode $i$.

The modal damping ratios can be determined from examining the rate of decay of each vibration mode during the system's response to an initial deflection. The modal matrix is then used to transform the damping matrix into the original coordinates 


$$
\mathrm{C}=\mathrm{PC}_{n} \mathrm{P}^{\mathrm{T}}
$$

Once the damping matrix is obtained, all the matrix elements of Eq. (2.1) are known and the mathematical model of the pump is complete.

\subsection{Calculation of Resonance Frequencies and Mode Shapes}

Before calculating the resonance frequencies and mode shapes of the system of equations given by Eq. (2.1), the 11 second-order differential equations defined by Eq. (2.1) are converted into 22 first-order differential equations. Substituting $Z_{2 i-1}=X_{i}$ and $Z_{2 i}=X_{i}, i=1$ to 11 into Eq. (2.1) eliminates all the second derivatives and forms 11 additional equations. The 22 first-order equations of motion may be written by using the matrix equation

$$
\dot{\mathbf{Z}}=\mathbf{A} \mathbf{Z}+\hat{\mathbf{F}}
$$

where $\hat{\mathbf{F}}$ is the expanded applied force vector. The resonance frequencies and mode shapes of the system are the complex eigenvalues and eigenvectors of the matrix $A$

\subsection{Calculation of the Temporal Response to Initial Conditions or Applied Forces}

Simulation of the response of the motor-pump unit to initial conditions or to applied forces was performed by using the Advanced Continuous Simulation Language (ACSL) computer code. ${ }^{10}$ The ACSL code was used to calculate the temporal response at the mass-point locations by numerical integration of Eq. (2.9). The initial section of the program forms the mass, stiffness, and damping matrices; combines these to form matrix $\mathbf{A}$ of Eq. (2.9); and sets the initial acceleration, velocity, and position of each mass point. The dynamic section of the program calculates the acceleration, velocity, and position of each mass point for each time increment. The output from the simulation, which consists of the time, acceleration, velocity, and the position of selected mass points, is stored in ASCI text and binary plotting files.

The calculated output for any mass point simulates measured acceleration data assuming the dynamics of the accelerometer are such that they do not significantly affect the measurement. This assumption is valid for frequencies below $80 \%$ of the accelerometer resonance frequency. For example, the calculated acceleration of mass point 10 simulates the signal that would be obtained from an accelerometer mounted on the motor casing. By processing the calculated acceleration through an FFT, a frequency spectrum can be obtained for each mass point. If the simulation corresponds to free vibration, the frequency spectra can be used to determine the resonance frequencies and mode shape components for the corresponding mass points. Resonance frequencies correspond to the peaks in the frequency spectrum, and the relative magnitude of each mode shape component may be obtained from the appropriate Fourier-transformed displacement component. The relative angle between eigenvector components can be obtained by comparing the angles from the appropriate Fourier-transformed displacement components. This information is extracted from the frequency spectra by the frequency 
spectrum decomposition technique. This technique is described in Appendix $\mathrm{A}$, and a previous application to the analysis of nuclear power plant noise is also discussed. ${ }^{11,12}$

\subsection{APPLICATION OF NEURAL NETWORKS AS CLASSIFIERS/ INTERPOLATORS}

A neural network is a mathematical algorithm modeled loosely on the concept of its biological counterpart, the brain. Just as the brain contains a vast number of interconnected neurons, a neural network comprises interconnected neuronlike "processing elements." Each processing element is a simple processor which, like its biological counterpart, receives signals from neighboring elements and outputs a response based on the weighted sum of those inputs. In most neural network configurations, the processing elements are arranged in connected layers, and the number of processing elements per layer may range from a few to thousands.

A distinguishing feature of a neural network is that it is adaptable and must undergo a training phase prior to application. During training, the values of coefficients (i.e., weights) that control the relative effects of processing elements are iteratively adjusted to produce a more desirable response. This adjustment is analogous to the biological learning process in which the interconnection strengths between neurons are strengthened or weakened, depending on relative activation rates. In general, both artificial and biological neural systems are governed by Hebb's Law, ${ }^{13}$ which states that when two neurons consistently and simultaneously have a high output, the interconnection strength between the neurons is increased.

Numerous neural network algorithms exist, and it is beyond the scope of this paper to discuss or even summarize them. However, in general, they can be grouped into two broad categories, depending on whether they are trained from a combination of input and output data (supervised) or from input data only (unsupervised). Two of the better known unsupervised neural networks are the Kohonen network ${ }^{14}$ and the Carpenter/ Grossberg Adaptive Resonance Theory (ART) network. ${ }^{15}$ These algorithms organize input data into categories on the basis of a measure of similarity and, thus, perform cluster analysis much like well-known classical algorithms such as the K-Means. ${ }^{16}$

Supervised networks, on the other hand, produce output values rather than clusters of data. Thus, supervised network training requires a set of data that includes both input and corresponding output values. The network is trained to reproduce each output value for the corresponding input value; thus, when it is presented with an unknown input, the correct output results. The training is typically accomplished by adjusting the weights of the processing element interconnections by an amount proportional to the error (i.e., the difference between the correct output and the output actually produced by the network). If the error is large, then large adjustments are made.

Two main applications of supervised neural networks exist: pattern recognition and function approximation. In the first application, a network is trained to classify input vectors (patterns). Outputs are constrained to discrete values, each of which indicates assignment to a particular class. In the second application, also known as system identification, a neural network is trained to duplicate the functional process of a physical system or mathematical model. Outputs in this case may be continuous rather than discrete. The interest in neural networks for this latter purpose stems largely from the Stone-Weierstrass Theorem which, when generalized to neural networks, states that even 
with only one hidden layer (see later discussion of backpropagation), a neural network can approximate any continuous function. ${ }^{17}$ As a result, a theoretical basis exists to conclude that a neural network can be trained to duplicate the mathematical details of an arbitrarily complex mathematical system. An advantage is that the functional response of a complex system may be duplicated with no requirement for knowledge of the internal details of the system. A conventional competing approach is the use of polynomial curve fitting. However, some research has indicated that neural networks can be orders of magnitude more accurate than polynomial models, which tend to oscillate and may require an impractically large number of coefficients. ${ }^{18}$

\subsection{Backpropagation Network}

The backpropagation model is the most widely used supervised neural network model and is used for the system identification in this work. This network is characterized by three or more layers of processing elements comprising an input buffer layer, at least one "hidden" layer, and an output layer. A conceptual example depicting $L$ hidden layers is shown in Fig. 2.2. Figure 2.3 shows a single processing element (element $j$ in layer $l$ ) where $x_{j}^{[n]}$ is the output $2 \mathrm{~s}$ given by

$$
x_{j}^{[n]}=g\left(S_{j}^{[!]}\right)
$$

where $g$ is a nonlinear transfer function that prevents signal saturation. $S_{j}^{[\eta}$ is the weighted sum of the outputs from the next lower layer and is given by

$$
S_{j}^{[n]}=\sum_{i} w_{j, i}^{[n} x_{i}^{[l-1]}
$$

The nonlinear transfer function is usually a hyperbolic tangent defined by

$$
g(z)=\frac{e^{z}-e^{-z}}{e^{z}+e^{-z}}
$$

or the sigmoid function defined by

$$
g(z)=\frac{1}{1+e^{-z}}
$$

A backpropagation network is trained by modifying the interconnection weights to minimize a global error function given by 


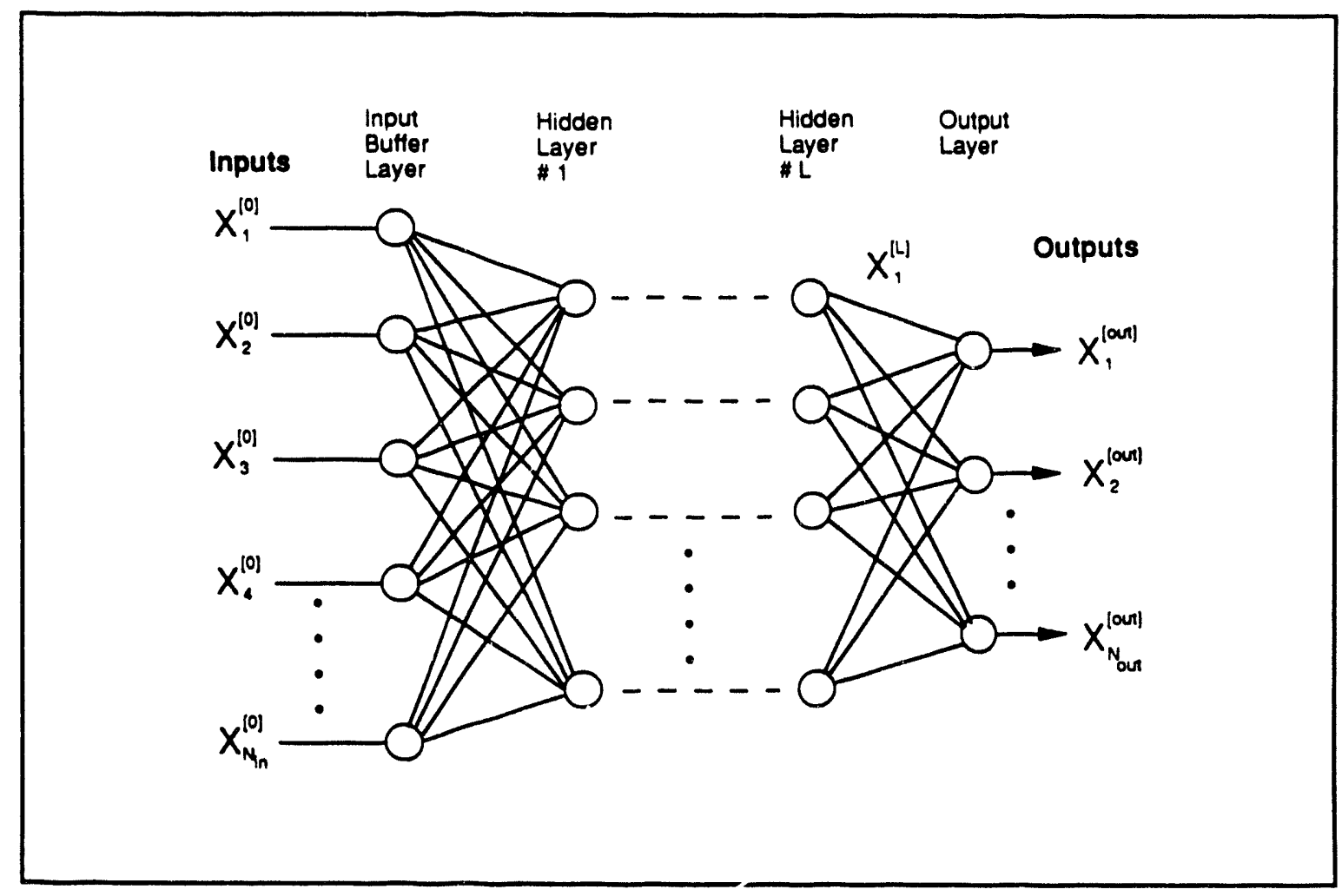

Fig. 2.2. Generalized backpropagation neural network with $L$ hidden layers.

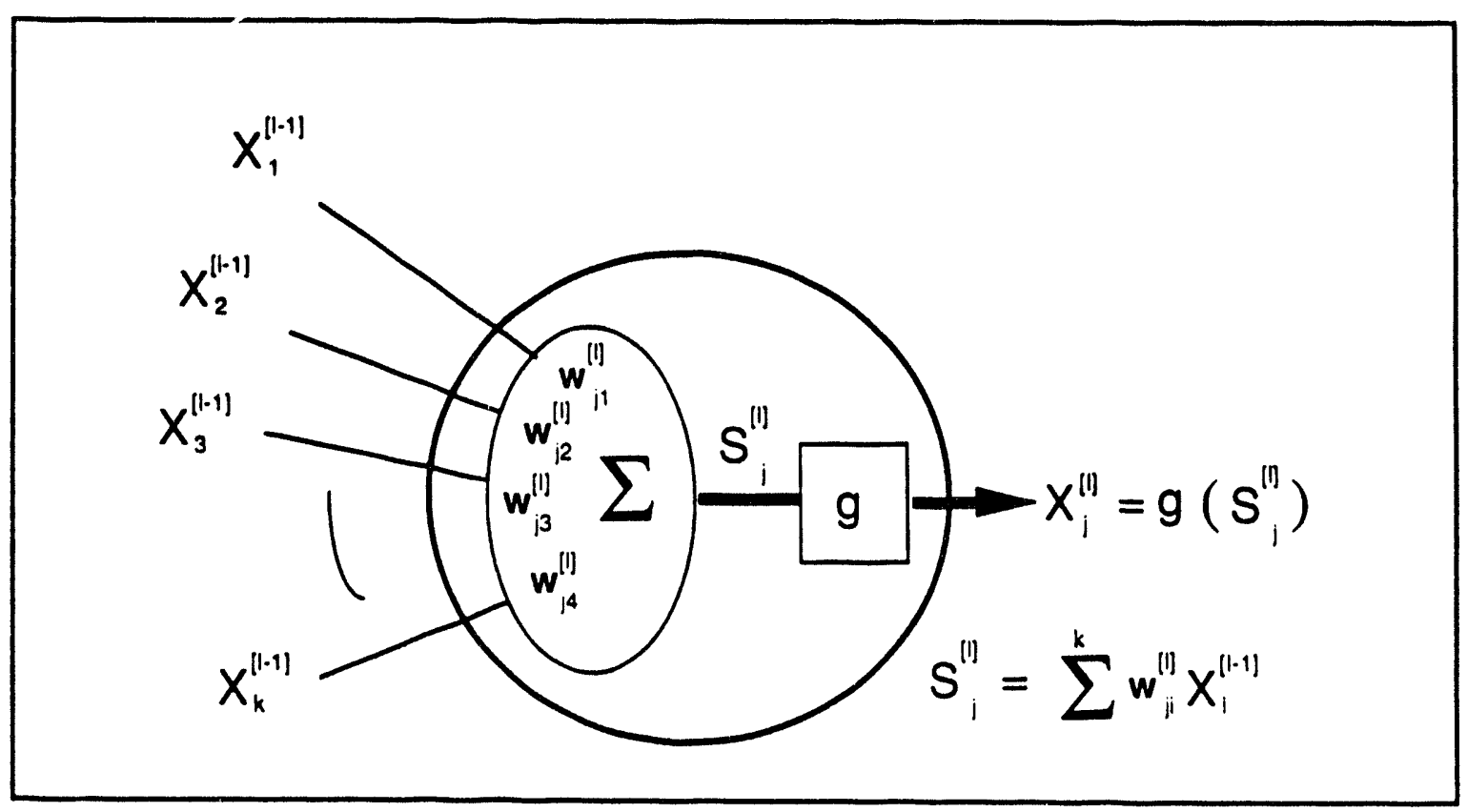

Fig. 23. Neural network processing element number $j$ in hidden layer $i$. 


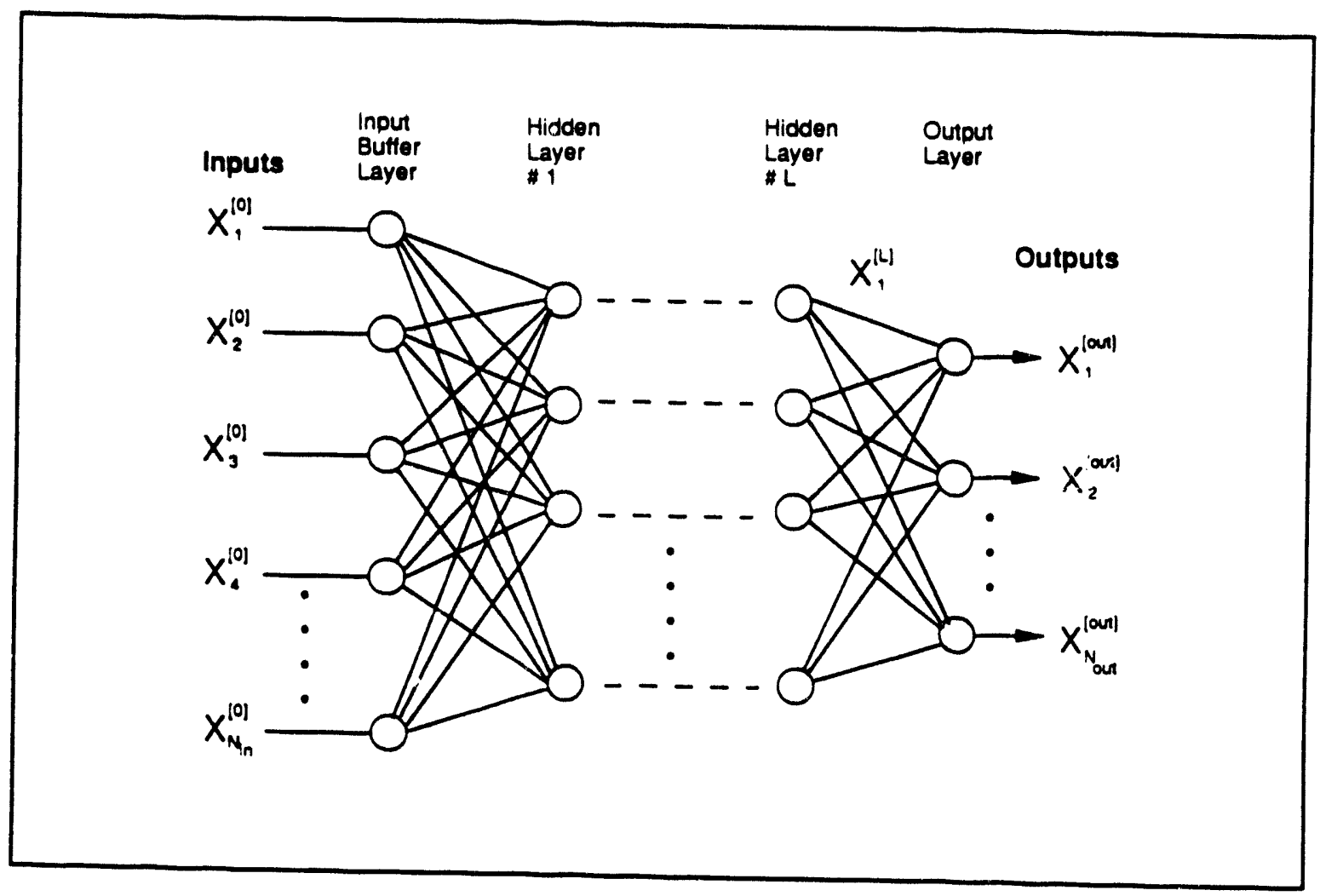

Fig. 22 Generalized backpropagation neural networt with $L$ hidden layers.

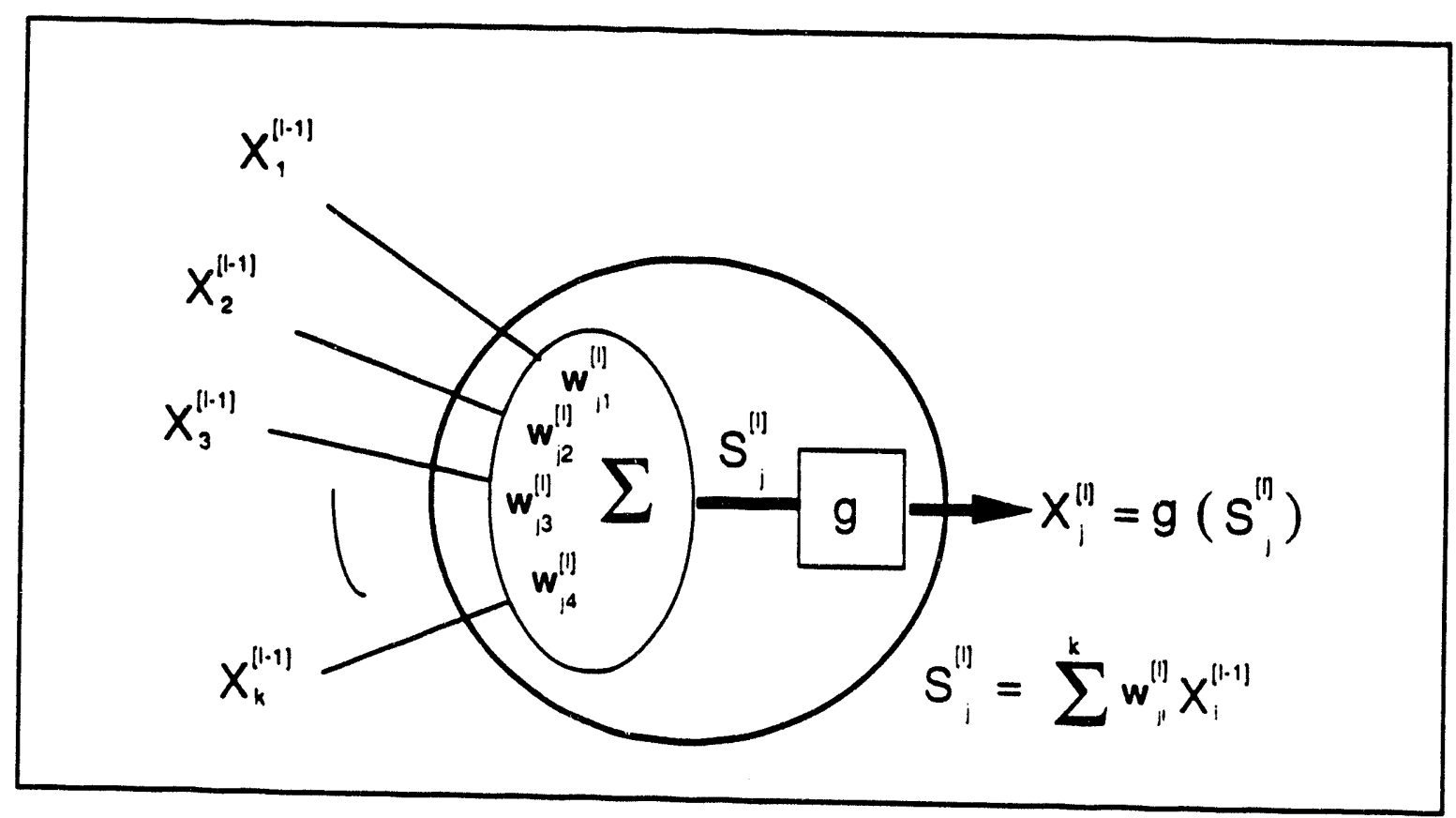

Fig. 23. Neural network processing element number $j$ in hidden layer $i$. 


$$
E=\frac{1}{2} \sum_{k=1}^{N}\left(y_{k}-x_{k}^{\text {[out] }}\right)^{2},
$$

where the $y_{k}$ values refer to the $N_{\text {out }}$ vector components of a "desired" output, that is, an output vector from the training set. The $x_{k}^{\text {[lut] }}$ are vector components produced by the network at the output iayer. It can be shown that the above global error decreases if the weights are modified according to the following rule, which uses the gradient descent approach:

$$
\Delta w_{j, i}^{[\eta]}=A e_{j}^{[\eta]} x_{i}^{[l-1]}
$$

where $\mathrm{A}$ is a constant and $e_{j}^{[\eta}$ is a local error at processing element $j$ in level $l$ and is defined by

$$
e_{j}^{[\eta]}=\frac{-\partial E}{\partial S_{j}^{[]}}
$$

which, upon substitution for $E$ and $S_{j}^{[\eta}$, results in

$$
e_{j}^{[n]}=g^{\prime}\left(S_{j}^{[n]}\right) \sum_{k}\left(e_{k}^{[1+11} w_{k j}^{[1+1]}\right)
$$

where the prime (') denotes differentiation. The above equation holds for all but the output layer, for which the error is given by

$$
e_{k}^{[\text {[out] }}=\left(y_{k}-x_{k}^{[\text {[out] }}\right) g^{\prime}\left(S_{k}\right)
$$

The constant $A(0<A<1)$ in Eq. (2.15) is known as the "Learning Coefficient." It has been heuristically shown that if $A$ is decreased during the learning phase, convergence is optimized. From Eqs. (2.10) and (2.11), we observe that data flow is in the feedforward direction from input to output. However, Eqs. (2.17) and (2.18) show that error information required to adjust the interconnection weights is propagated in a backward direction from output to input. Hence, the name "backpropagation" has been adopted.

One issue that has attracted concern regarding gradient descent methods is that of local minima. That is, how can one be certain that the minimum error obtained is indeed a global minimum? It has been shown that for a local minimum to exist, all the weights must be such that any change in these weights will increase the error. ${ }^{19}$ The indication is that the probability of a local minimum decreases for a specific data set as the number of weights is increased. Thus, if a local minimum is suspected, the addition of nodes to the hidden layer should eliminate the local minimum. A further indication is that local minima present more of a theoretical problem than one that actually occurs in practice. 


\section{PHASE I: APPLYING THE DIAGNOSTIC METHOD TO COMPUTER-SIMULATED DATA}

This chapter describes the development of software tools and the application of the diagnostic method to computer-simulated data. The computer simulation was intended to address the following questions:

1. What effect does the training set composition and size have on the accuracy of the model parameter estimates made by the neural network?

2. Is the formation of the training set or the neural network training so computationally intensive that the diagnostic method is impractical?

3. Are eigenvalues and eigenvector components a practical choice for forming the neural network training set, and is the information contained in these values sufficient for the diagnostic method to accurately predict model parameters?

4. Can information equivalent to the eigenvalues and eigenvector components used to form the training set be easily extracted from vibration spectra?

5. Can the trained neural network solve the "inverse problem," that is, can the neural network $b \approx$ used to accurately estimate the model parameters that correspond to a given set of eigenvalues and eigenvector components (resonance frequencies and mode shape components)?

With the use of computer-simulated data, a direct comparison of the estimated and known model parameters can be used to evaluate the accuracy of the neural network interpolation. The use of computer-simulated data also allows an examination of the effect of the frequency spectrum decomposition on diagnosis accuracy.

The formation of the neural network training sets is described in Sect. 3.1. The application of the NeuralWorks Professional II + code to perform the neural network calculations is described in Sect. 3.2. The application of the diagnostic method to computer-simulated data is described in Sect. 3.3, and a discussion of the results is presented in Sect. 3.4.

\subsection{FORMATION OF THE TRAINING SETS USED DURING PHASE I}

The members of the training sets used during Phase I were selected so the effects of the spacing between the members in the training set and the effects of the number and type of model output values on neural network prediction accuracy could be examined. The number of model output values determines the number of nodes in the neural network input and hidden layers. The spacing between the model input values affects the neural network prediction accuracy because closer input value spacings result in neural network interpolation over a narrower range during the recall phase. Because each training set used in this investigation spans the same range of input and output values, the 
number of members contained in the training set increases as the spacing between members decreases. The effect of the number of spring rates used during the generation of the training set $n$ and the number of variations of spring rate $j, i_{j}$ on the number of cases contained in each training set $N$ is given by

$$
N=\prod_{i=1}^{n} i_{j}
$$

Thus, the number of members contained in the training set increases rapidly with either the number of spring rates adjusted during the generation of the training set or with the number of variations of each spring rate.

The number of spring rates adjusted during the formation of the training sets (i.e., the number of neural network output parameters) was limited to three; the two mounting spring rates $K_{m m}$ and $K_{m p}$ and the stiffness of the shaft between mass points 4 and $5, E I_{45}$. Limiting the number of spring rates adjusted during the investigation allowed the effects of training set member spacing on neural network performance to be investigated thoroughly without forming overwhelmingly large neural networks or training sets. Three sets of spring rates were used in the Phase I portion of the investigation. The numerical values of $K_{m m}, K_{m p}$, and $E I_{45}$ for each spring rate set are listed in Table 3.1. Training set input parameters were selected after examining the effect of changing the spring rates on the calculated resonance frequencies and mode shapes. This examination showed which modes and mode features were most affected by changing $K_{m m}, K_{m p}$, and $E I_{45}$.

The undamped natural frequencies and mode shapes calculated by using three sets of spring rates that span the range of those used in the Phase I portion of this investigation are shown in Appendix B. The eleven modes are designated by the letters $A$ to $K$ in the Appendix B figures. Examination of the mode shapes calculated over the range of spring rates shows that the characteristics of modes $A, C, E$, and $F$ are affected significantly by changes in the values of $K_{m m}, K_{m p}$, and $E I_{45}$. These modes are characterized by the following features:

Mode A Mode A is characterized by relatively large mode shape components corresponding to mass points 5,6 , and 8 . The mode shape components corresponding to mass points 5 and 6 are in-phase, and the mode shape components corresponding tc mass points 5 and 8 are out-of-phase. The mode shape components corresponding to mass points 10 and 11 have a value of virtually zero.

Mode C Mode $\mathrm{C}$ is characterized by relatively large mode shape components corresponding to mass points 5,6 , and 8 . The mode shape components corresponding to mass points 5 and 6 are in-phase, and the mode shape components corresponding to mass points 5 and 8 are also in-phase. The mode shape components corresponding to mass points 10 and 11 have a value of virtually zero. 
Table 3.1. Spring rate values used in the Phase I training sets

\begin{tabular}{|c|c|c|}
\hline $\begin{array}{c}K_{m \mathrm{~m}} \\
{\left[\mathrm{lb} / \mathrm{in} .^{2}(\mathrm{~N} / \mathrm{m})\right]}\end{array}$ & $\begin{array}{c}K_{m p} \\
{\left[1 \mathrm{f}_{\mathrm{p}} \mathrm{in}^{2}(\mathrm{~N} / \mathrm{m})\right]}\end{array}$ & $\begin{array}{c}E I_{45} \\
{\left[1 \mathrm{~b}_{\mathrm{f}} \cdot \mathrm{in}^{2}\left(\mathrm{~N} \cdot \mathrm{m}^{2}\right)\right]}\end{array}$ \\
\hline \multicolumn{3}{|c|}{ Set 1,27 combinations } \\
\hline $1 \times 10^{5}\left(1.75 \times 10^{7}\right)$ & $1 \times 10^{5}\left(1.75 \times 10^{7}\right)$ & $1 \times 10^{6}\left(2.87 \times 10^{3}\right)$ \\
\hline $5 \times 10^{5}\left(8.76 \times 10^{7}\right)$ & $5 \times 10^{5}\left(8.76 \times 10^{7}\right)$ & $5 \times 10^{6}\left(1.44 \times 10^{4}\right)$ \\
\hline $1 \times 10^{6}\left(1.75 \times 10^{8}\right)$ & $1 \times 10^{6}\left(1.75 \times 10^{8}\right)$ & $1 \times 10^{7}\left(2.87 \times 10^{4}\right)$ \\
\hline \multicolumn{3}{|c|}{ Set 2,125 combinations } \\
\hline $1 \times 10^{5}\left(1.75 \times 10^{7}\right)$ & $1 \times 10^{5}\left(1.75 \times 10^{7}\right)$ & $1 \times 10^{6}\left(2.87 \times 10^{3}\right)$ \\
\hline $2.5 \times 10^{5}\left(4.38 \times 10^{7}\right)$ & $2.5 \times 10^{5}\left(4.38 \times 10^{7}\right)$ & $2.5 \times 10^{6}\left(7.20 \times 10^{3}\right)$ \\
\hline $5 \times 10^{5}\left(8.76 \times 10^{7}\right)$ & $5 \times 10^{5}\left(8.76 \times 10^{7}\right)$ & $5 \times 10^{6}\left(1.44 \times 10^{4}\right)$ \\
\hline $7.5 \times 10^{5}\left(1.313 \times 10^{8}\right)$ & $7.5 \times 10^{5}\left(1.313 \times 10^{8}\right)$ & $7.5 \times 10^{6}\left(2.15 \times 10^{4}\right)$ \\
\hline $1 \times 10^{6}\left(1.75 \times 10^{8}\right)$ & $1 \times 10^{6}\left(1.75 \times 10^{8}\right)$ & $1 \times 10^{7}\left(2.87 \times 10^{4}\right)$ \\
\hline \multicolumn{3}{|c|}{ Set 3,343 combinations } \\
\hline $1 \times 10^{5}\left(1.75 \times 10^{7}\right)$ & $1 \times 10^{5}\left(1.75 \times 10^{7}\right)$ & $1 \times 10^{6}\left(2.87 \times 10^{3}\right)$ \\
\hline $1.66 \times 10^{5}\left(2.91 \times 10^{7}\right)$ & $1.66 \times 10^{5}\left(2.91 \times 10^{7}\right)$ & $1.66 \times 10^{6}\left(4.76 \times 10^{4}\right)$ \\
\hline $3.33 \times 10^{5}\left(5.83 \times 10^{7}\right)$ & $3.33 \times 10^{5}\left(5.835 \times 10^{7}\right)$ & $3.33 \times 10^{6}\left(9.56 \times 10^{3}\right)$ \\
\hline $5 \times 10^{5}\left(8.76 \times 10^{7}\right)$ & $5 \times 10^{5}\left(8.76 \times 10^{7}\right)$ & $5 \times 10^{6}\left(1.44 \times 10^{4}\right)$ \\
\hline $6.66 \times 10^{5}\left(1.17 \times 10^{8}\right)$ & $6.66 \times 10^{5}\left(1.17 \times 10^{8}\right)$ & $6.66 \times 10^{6}\left(1.91 \times 10^{4}\right)$ \\
\hline $8.33 \times 10^{5}\left(1.46 \times 10^{8}\right)$ & $8.33 \times 10^{5}\left(1.46 \times 10^{8}\right)$ & $8.33 \times 10^{6}\left(2.39 \times 10^{4}\right)$ \\
\hline $1 \times 10^{6}\left(1.75 \times 10^{8}\right)$ & $1 \times 10^{6}\left(1.75 \times 10^{8}\right)$ & $1 \times 10^{7}\left(2.87 \times 10^{4}\right)$ \\
\hline
\end{tabular}

Mode $\mathrm{E} \quad$ Mode $\mathrm{E}$ is characterized by a significant mode shape component corresponding to mass point 10 . The mode shape component corresponding to mass point 11 is virtually zero.

Mode $\mathrm{F} \quad$ Mode $\mathrm{F}$ is characterized by a significant mode shape component corresponding to mass point 11 . The mode shape component corresponding to mass point 10 is virtually zero.

Also, over the range of spring rates examined, modes $\mathrm{A}, \mathrm{C}, \mathrm{E}$, and $\mathrm{F}$ correspond to one of the 9 lowest resonance frequencies. Thus, the calculated results indicate that modes $\mathrm{A}, \mathrm{C}, \mathrm{E}$, and $\mathrm{F}$ will show significant changes caused by variations in $K_{m m}, K_{m p}$, and $E I_{45}$ and that these modes can be identified on the basis of the mode shape components corresponding to mass points $5,8,10$, and 11 . 
The members of the training sets used during the Phase I portion of this investigation are summarized in Table 3.2. The training sets differ in the number of spring rate combinations contained in the training sets and in the number and type of neural network input parameters. Training sets 1,2 , and 3 used only the resonance frequencies of modes $A, C, E$, and F (presented in that order) as input. Training sets 4,5 , and 6 use the resonance frequencies and normalized mode shape components corresponding to mass points $5,8,10$, and 11 for modes $A, C, E$, and $F$ as input. Training sets 7,8 , and 9 use the resonance frequencies and normalized mode shape components of mass points 5,8 , 10 , and 11 corresponding to the 9 lowest resonance frequencies as input. Training sets 1 through 6 order the neural network input parameters on the basis of mode and, thus, require some preprocessing of the input parameters. This preprocessing removes those modes that are less sensitive to changes in $K_{m m}, K_{m p}$, and $E I_{45}$, resulting in a relatively small neural network. Training sets 7,8 , and 9 contain resonance frequency and mode shape information corresponding to the 9 lowest resonance frequencies. This approach removes the need to preprocess the input parameters but results in larger neural networks.

Table 3.2. Training sets used in the Phsse I portion of the investigation

\begin{tabular}{crrr}
\hline Training set & Spring rate set & Ordered by mode & Mode shape components \\
\hline 1 & 1,27 components & Yes, modes A, C, E, and F & None, frequencies only \\
2 & 2,125 components & Yes, modes A, C, E, and F & None, frequencies only \\
3 & 3,343 components & Yes, modes A, C, E, and F & None, frequencies only \\
4 & 1,27 components & Yes, modes A, C, E, and F & $5,8,10$, and 11 \\
5 & 2,125 components & Yes, modes A, C, E, and F & $5,8,10$, and 11 \\
6 & 3,343 components & Yes, modes A, C, E, and F & $5,8,10$, and 11 \\
7 & 1,27 components & No, first 9 modes & $5,8,10$, and 11 \\
8 & 2,125 components & No, first 9 modes & $5,8,10$, and 11 \\
9 & 3,343 components & No, first 9 modes & $5,8,10$, and 11 \\
\hline
\end{tabular}

\subsection{CREATION AND TRAINING OF THE NEURAL NETWORK}

All neural network development in this work was performed by using the NeuralWorks Professional II/PLUS code distributed by NeuralWare, Inc., of Pittsburgh. ${ }^{20}$ This package offers an extensive selection of neural network paradigms. However, as was discussed in Sect. 2.2, the backpropagation algorithm appeared the most suitable for our application. We found, after some experimentation, that only one hidden layer was required in each network representation and little could be gained by increasing this number. Each network had three outputs corresponding to the spring rates $K_{m m}, K_{m p}$, and $E I_{45}$. The number of inputs included the resonance frequency values and the mode shape 
components for each resonance frequency. Thus, for training sets 1,2 , and 3 , which use four resonance frequencies and no mode shapes, only four inputs were used. For sets 4 , 5 , and 6, which use four resonance frequencies and four mode shapes (per frequency), 20 inputs were required. Similarly, for sets 7, 8, and 9, which have nine frequencies and four mode shapes, 45 inputs were required. Thus, the complexity of the neural networks used in this work varied from a 4 - to a 45-dimensional input.

No technique is universally accepted for determining the ideal number of nodes to include in the hidden layer of a backpropagation network. However, we found, through trial and error, that a suitable number of hidden layer nodes was $-50 \%$ of the input dimension (provided that number remained greater than the number of output nodes). A larger number only complicated the network and did not improve either convergence rate or the final accuracy; a smaller number in some cases degraded the final results. On this basis, the number of hidden nodes for the three groupings $1-3,4-6$, and 7-9 were 3,10 , and 20 respectively.

Additional factors that must be considered in the development of a backpropagation network are the nonlinear transfer function used and the variation of the learning rule incorporated. For our work, the hyperbolic tangent gave better results than the sigmoid function and was used in all cases. Network learning was achieved by using the cumulative delta rule, a version of the gradient descent rule discussed in Sect. 2.2. The weight changes (delta weights) are averaged over a specified number of training set member presentations to the network before the weights are actually updated. The number of results accumulated before the update is referred to as the "epoch." Thus, for the standard gradient descent approach in which the weights are updated after each presentation, the epoch is 1 . The advantage of an epoch $>1$ is that oscillatory results from individual input-desired output pairs are smoothed out. The disadvantages are that more presentations are required for each weight update, and if the epoch is too large, the weight changes may average to a very small value, resulting in slow convergence. For the cased we examined, the epoch size appeared to have a marginal effect on the ultimate convergence of the network. In general, we found that an epoch size that was several percentage of the training set size resulted in a short training time.

Table 3.3 summarizes the above discussion describing network parameters for the nine training sets considered. Table 3.3 shows also the number of iterations that were used for training each network. Errors are discussed in the following section.

\subsection{RESULTS OBTANNED FROM APPLYING THE DIAGNOSTIC METHOD TO COMPUTER-SIMULATED DATA}

This section presents the results obtained from applying the diagnostic method to computer-simulated data. The use of the diagnostic method to estimate model spring rates from calculated eigenvalue and eigenvector components is presented first, followed by the results obtained from applying the method to computer-simulated vibration spectra. 
Table 3.3. Neural network parameters

\begin{tabular}{cccccc}
\hline $\begin{array}{c}\text { Training } \\
\text { set }\end{array}$ & $\begin{array}{c}\text { Nodes in } \\
\text { input layer }\end{array}$ & $\begin{array}{c}\text { Nodes in } \\
\text { hidden layer }\end{array}$ & $\begin{array}{c}\text { Nodes in } \\
\text { output layer }\end{array}$ & $\begin{array}{c}\text { Histories } \\
\text { (in thousands) }\end{array}$ & $\begin{array}{c}\text { Epoch } \\
\text { size }\end{array}$ \\
\hline 1 & 4 & 3 & 3 & 30 & 9 \\
2 & 4 & 3 & 3 & 30 & 25 \\
3 & 4 & 3 & 3 & 30 & 50 \\
4 & 20 & 10 & 3 & 20 & 9 \\
5 & 20 & 10 & 3 & 20 & 25 \\
6 & 20 & 10 & 3 & 20 & 25 \\
7 & 45 & 20 & 3 & 5 & 9 \\
8 & 45 & 20 & 3 & 30 & 25 \\
9 & 45 & 20 & 3 & 40 & 50 \\
\hline
\end{tabular}

\subsubsection{Prediction of Spring Rates From Calculated Eigenvalue and Eigenvector Information}

The ability of the neural network to reproduce the training set output, given the training set input, is shown in Figs. 3.1, 3.2, and 3.3. The difference between the known and predicted output is a measure of the effectiveness of the neural network training. These figures show results obtained by applying a neural network trained by using training set 6; this particular neural network/training set combination was selected because it produced the most accurate results. In these figures, examples of perfect agreement between the known and estimated values fall on the diagonal line. For the neural network reproducing the spring rates used in training set 6 , the average absolute error and standard deviation of the three estimated spring rates is 3.6 and $3.1 \%$ respectively.

The capability of the neural network to generalize over the training set (i.e., to interpolate between the spring rate values used in the training set) is shown in Figs. 3.4, 3.5, and 3.6. A test set was formed by using calculated results for spring rates between those used in the training set. The overall absolute error is $3.2 \%$ and the standard deviation is $2.7 \%$.

The effect of the training set size on the accuracy of the neural network estimate of spring rates not included in the training set is shown in Fig. 3.7. These results show that for each of the training set types, the accuracy of the neural network spring rate estimate improves as the number of training set members increases. This improvement occurs because the greater number of training set members reduces the range over which the neural network must interpolate to estimate the spring rate values. The rate of improvement decreases as the number of training set members increases.

\subsubsection{Prediction of Spring Rates from Calculated Vibration Spectra}

The ACSL computer code was used to calculate the model mass-point deflections caused by an initial deflection of mass point 1 . The calculated deflection data were 


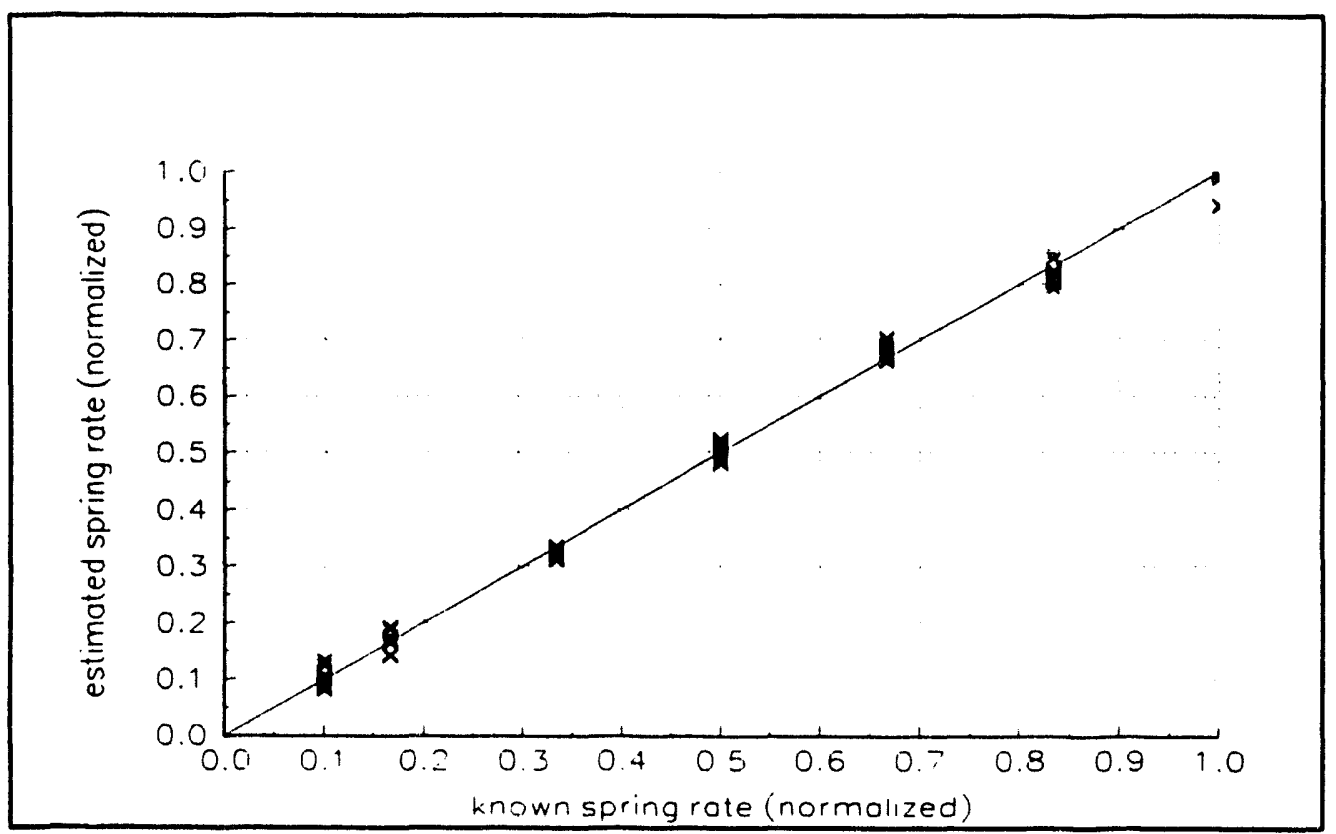

Fig. 3.1. Comparison of the known and estimated spring rates for the values of $K_{m m}$ used in the training set.

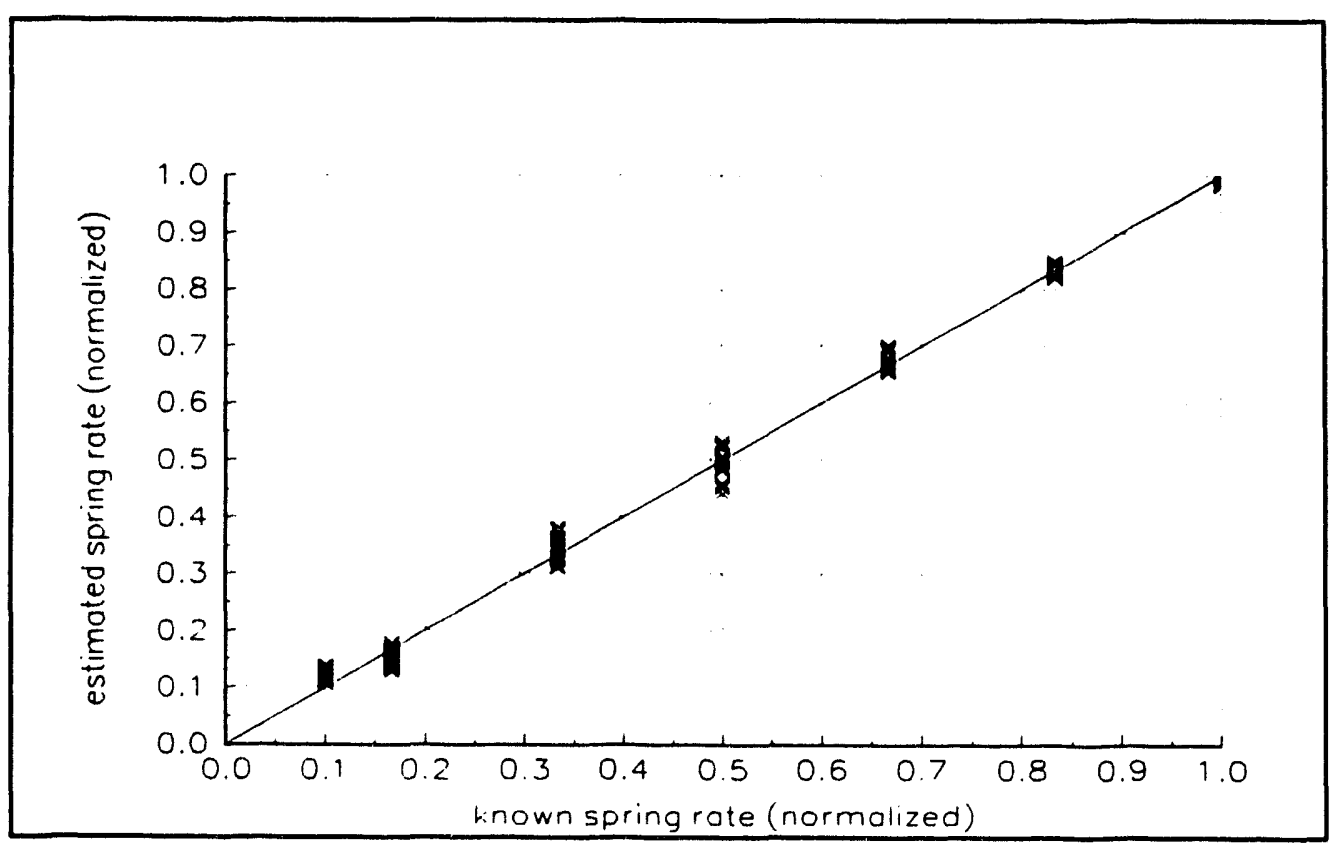

Fig. 3.2. Comparison of the known and estimated spring rates for the values of $K_{m p}$ used in the training set. 


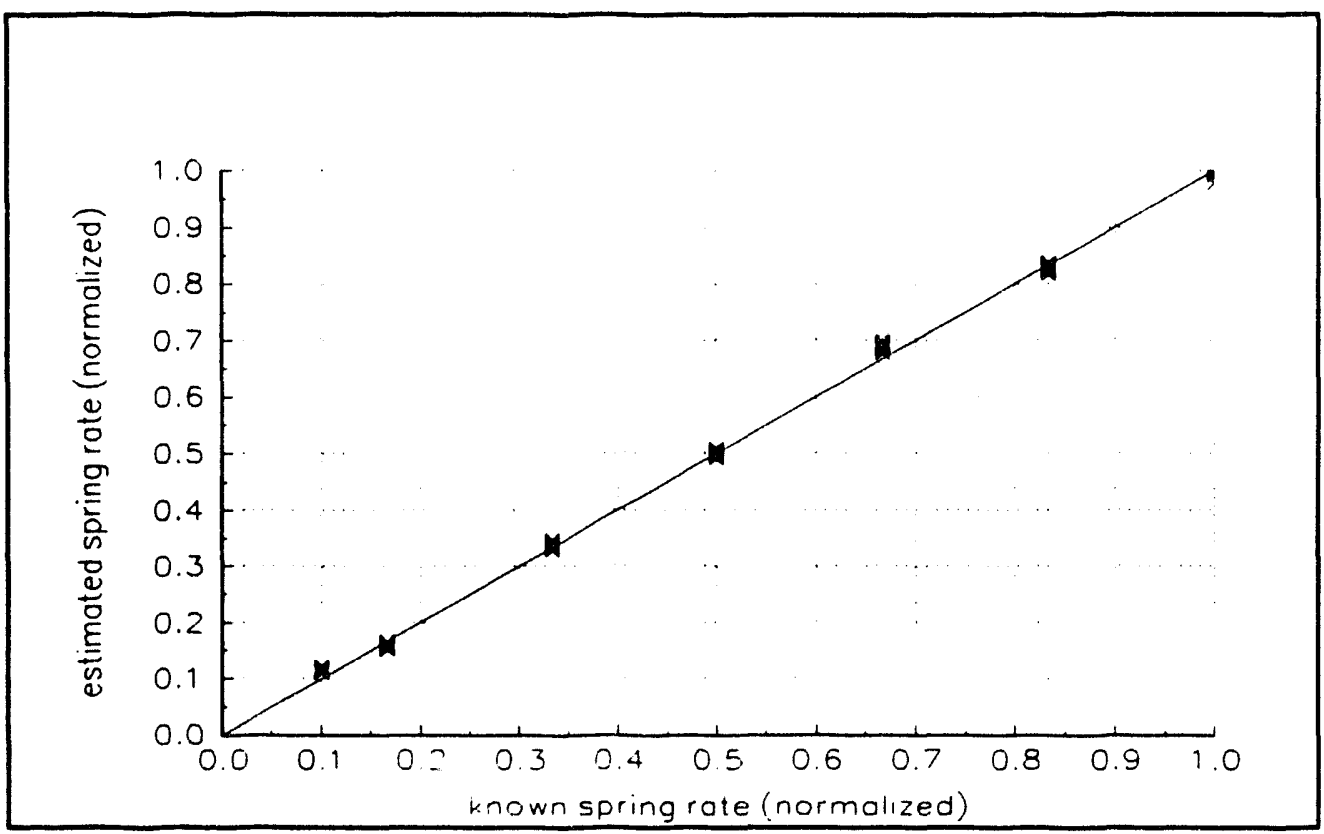

Fig. 3.3. Comparison of the known and estimated spring rates for the values of $E I_{\text {is }}$ used in the training set.

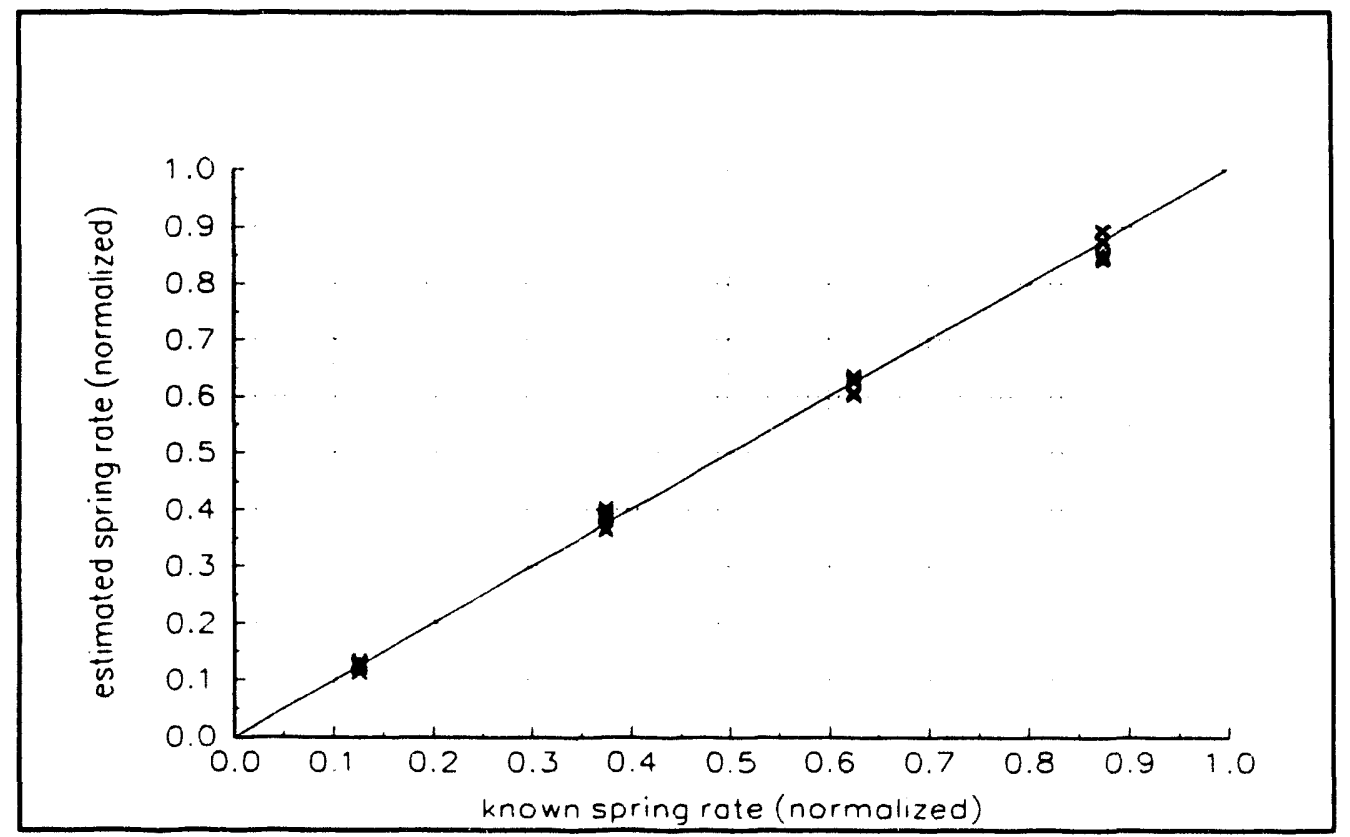

Fig. 3.4. Comparison of the known and estimated spring rates for values of $K_{m m}$ between those used in the training set. 


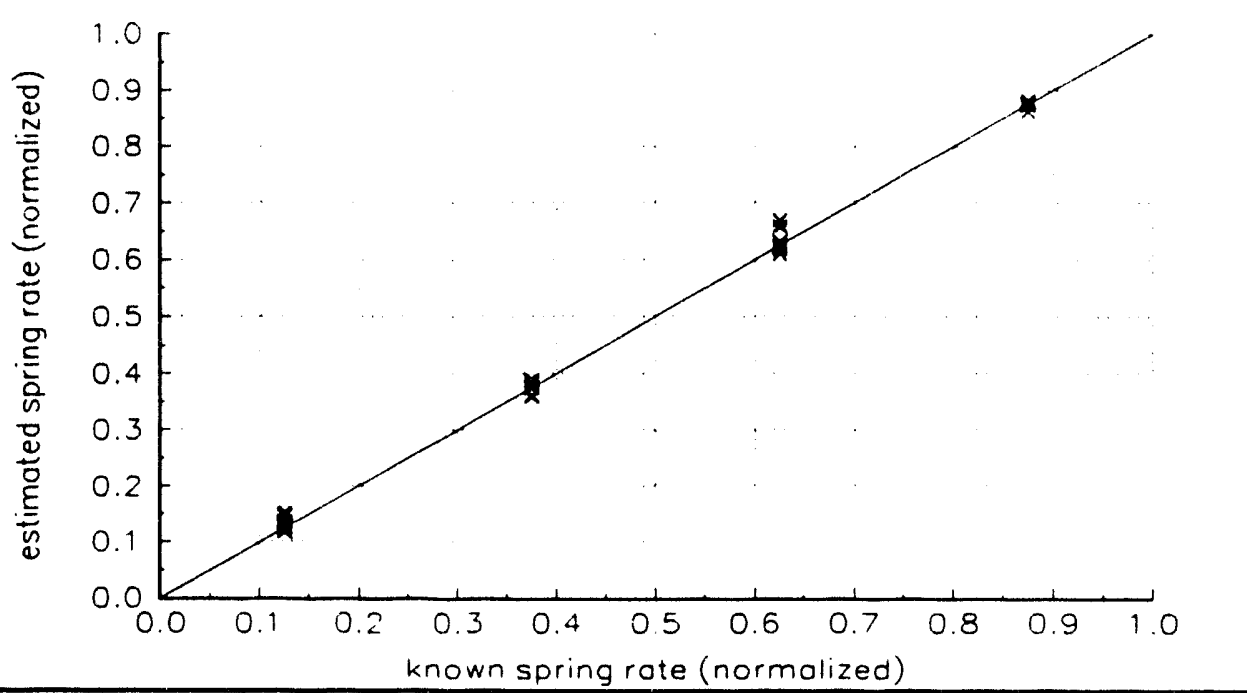

Fig. 3.5. Comparison of the known and estimated spring rates for values of $K_{m p}$ between those used in the training set.

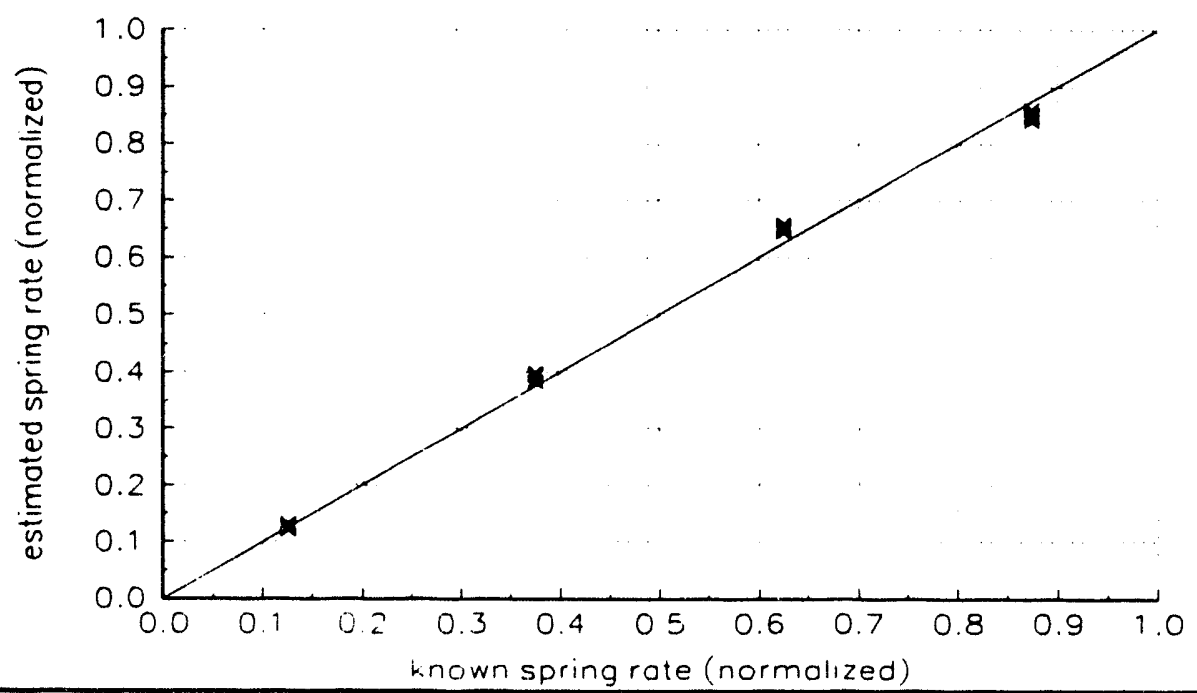

Fig. 3.6. Comparison of the known and estimated spring rates for values of $E I_{45}$ between those used in the training set. 


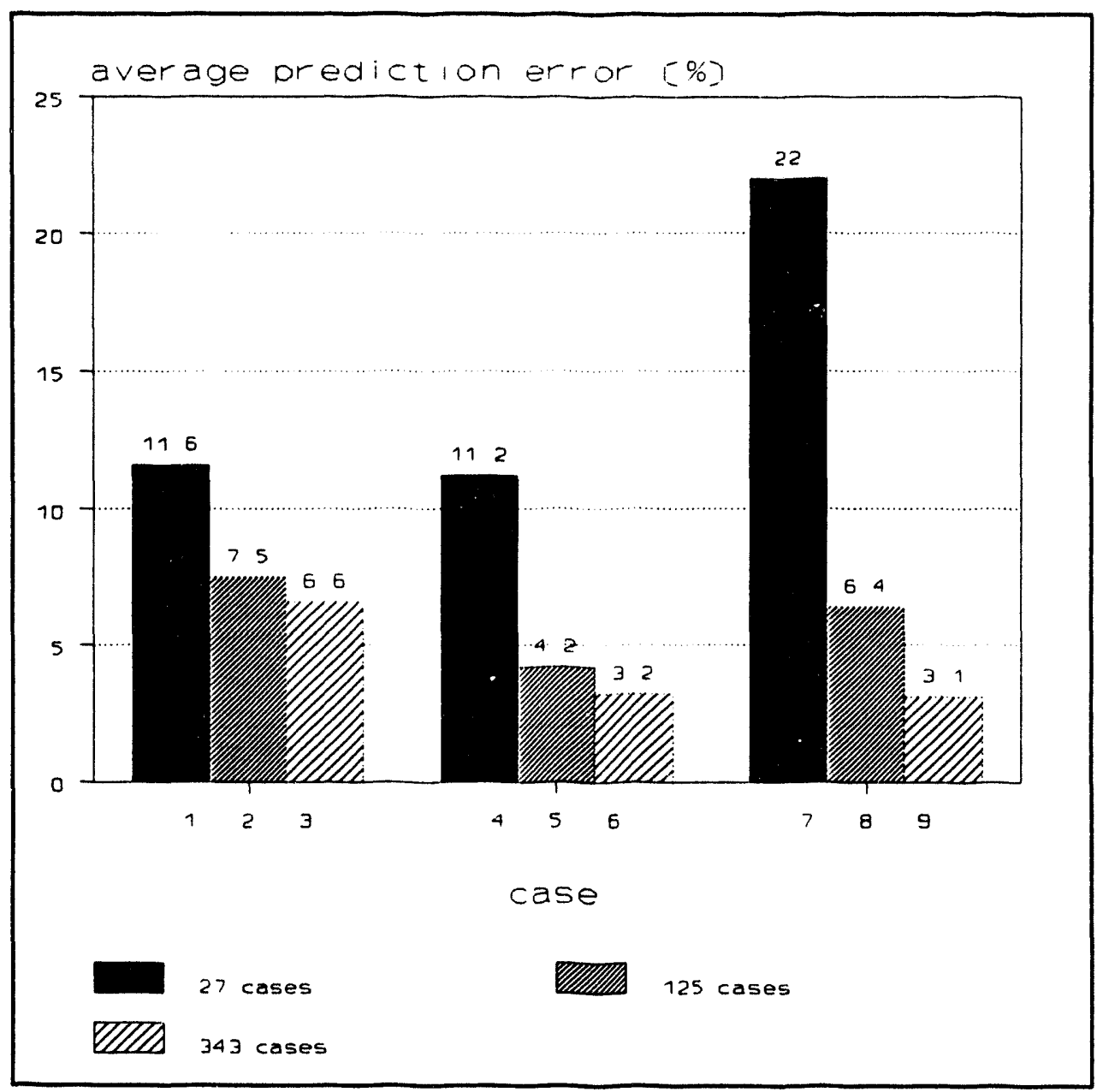

Fig. 3.7. Effect of the number of training set members on neural network estimation accuracy for the 3 training set types used during Phase I.

Fourier transformed into the frequency domain, and the neural network input data (i.e., resonant frequencies and mode shape amplitudes) were manually extracted from the frequency spectra. Comparison of the known and estimated spring rates indicates how well the technique can be applied to spectral data.

The response of the system to a $1-$ in. $(2.54-\mathrm{cm})$ initial displacement of mass point 1 was calculated for a simulation time span of $4.5 \mathrm{~s}$ with the mass point displacements recorded at time intervals of $0.001 \mathrm{~s}$. The results of this calculation were used to form a 4096-line Fourier transform to be performed with a frequency increment of $-0.25 \mathrm{~Hz}$ and a maximum frequency of $250 \mathrm{~Hz}$. A fourth-order Butterworth filter with a $200-\mathrm{Hz}$ cutoff frequency was applied to the time-domain data before the displacement of mass points 5 , 8,10 , and 11 were transformed into the frequency domain.

The amplitude and phase of the spectra at each resonance frequency were used to form the mode shape components used as neural network input. To form the neural 
network input, the resonance frequencies were determined for each spectrum. The amplitude and phase of mass points 5, 8, 10, and 11 were used to determine the mode type (i.e., mode A, B, C, . ., K) and the appropriate peak amplitudes (normalized so the largest amplitude for each mode equals 1.0) and phases were used to form the normalized mode shape components. Thus, the neural network input consisted of the resonance frequencies and normalized mode shapes of modes A, C, E, and F. This combination of input parameters is the same as the neural network input contained in training sets 4,5 , and 6 (see Table 3.2).

A neural network trained by employing training set 6 was used to estimate the model spring rates from the extracted modal data. The known and estimated spring rates for 9 spring rate combinations are listed in Table 3.4. These results show average errors of $\sim 8 \%$ for the estimated values of $K_{m m}$ and $K_{m p}$ and an average error of $\sim 12 \%$ for the estimated values of $E I_{45}$. The absolute level of the error in the estimated values is approximately the same for most of the estimates $\left(\sim 2 \times 10^{4}\right)$, thus causing the error for the cases with low spring rate values (of which there are 7 ) to have relatively large errors when expressed in percentages. Thus, the estimation error appears unusually large for this set of cases because it contains many relatively small spring rate values. The spring rate estimation accuracy is judged to be roughly equivalent to that obtained by using calculated eigenvalues and eigenvector components, with any additional accuracy loss being attributed to the resonant frequency and mode shape errors associated with obtaining the discrete frequency spectra from the Fourier transform of the time domain data.

\subsection{DISCUSSION OF RESULTS}

The results from the computer simulation show that the trained neural network can be used to estimate model parameters to within $3 \%$, given appropriate eigenvalue/ eigenvector (resonance frequency and mode shape) information. Thus, the neural network can be used to solve the "inverse problem," provided the following conditions are met:

1. The relationship between the neural network input (the eigenvalue/eigenvector information) and the neural network output (the model parameters) must be single valued.

2. The values of the parameters used as neural network input must be significantly affected by changes in the neural network output parameters.

If the relationship between the neural network input and output is not single valued, the model parameter estimates will be inaccurate. Training sets 1,2 , and 3 show this effect. These training sets use only resonance frequencies as neural network input. Because some of the spring rate combinations in the training set have virtually identical resonance frequency values (the mode shape component values are different), one-to-one correspondence between neural network input and output does not exist. The results shown in Fig. 3.7 show that the model parameter estimates made by using neural networks trained with training sets 1,2 , or 3 are less accurate than those made by using neural networks trained with training sets 4 through 9 . The relationship between the neural network input and output is single valued in training sets 4 through 9 because these training sets include mode shape components. 
Table 3.4. Comparison of known spring rates with spring rates estimated from simulated spectral data

\begin{tabular}{|c|c|c|c|c|c|c|c|c|c|c|c|c|}
\hline \multirow[b]{3}{*}{ Case } & \multicolumn{6}{|c|}{ Known } & \multicolumn{6}{|c|}{ Estimated } \\
\hline & \multicolumn{2}{|c|}{$K_{m}$} & \multicolumn{2}{|c|}{$K_{m}$} & \multicolumn{2}{|c|}{$E I_{\omega s}$} & \multicolumn{2}{|c|}{$K$} & \multicolumn{2}{|c|}{$K_{-}$} & \multicolumn{2}{|c|}{$E I_{\omega}$} \\
\hline & $(\mathrm{lb}, \mathrm{fn}$.) & $(\mathrm{N} / \mathrm{m})$ & $(\mathrm{lb}, / \mathrm{n}$. $)$ & $(\mathrm{N} / \mathrm{m})$ & $\left(\mathrm{b}_{\mathrm{f}}+\mathrm{in}^{2}\right)$ & $\left(\mathrm{N} \cdot \mathrm{m}^{2}\right)$ & $(\mathrm{lb}, \mathrm{fin})$. & $(\mathrm{N} / \mathrm{m})$ & (lb/fin.) & $(N / m)$ & $\left(1 b_{f}-\right.$ in. $\left.^{2}\right)$ & $\left(\mathbf{N} \cdot \mathrm{m}^{2}\right)$ \\
\hline 1 & $1 \times 10^{5}$ & $1.75 \times 10^{7}$ & $1 \times 10^{5}$ & $1.75 \times 10^{7}$ & $1 \times 10^{6}$ & $2.87 \times 10^{3}$ & $1.26 \times 10^{5}$ & $221 \times 10^{7}$ & $1.17 \times 10^{5}$ & $205 \times 10^{7}$ & $1.31 \times 10^{6}$ & $3.76 \times 10^{3}$ \\
\hline 2 & $1 \times 10^{5}$ & $1.75 \times 10^{7}$ & $1 \times 10^{5}$ & $1.75 \times 10^{7}$ & $1 \times 10^{7}$ & $2.87 \times 10^{4}$ & $1.06 \times 10^{5}$ & $1.86 \times 10^{7}$ & $1.24 \times 10^{5}$ & $2.17 \times 10^{7}$ & $1.01 \times 10^{7}$ & $290 \times 10^{4}$ \\
\hline 3 & $1 \times 10^{5}$ & $1.75 \times 10^{7}$ & $1 \times 10^{6}$ & $1.75 \times 10^{8}$ & $1 \times 10^{6}$ & $287 \times 10^{3}$ & $1.26 \times 10^{5}$ & $2.21 \times 10^{7}$ & $9.73 \times 10^{5}$ & $1.70 \times 10^{2}$ & $1.29 \times 10^{6}$ & $3.70 \times 10^{3}$ \\
\hline 4 & $1 \times 10^{5}$ & $1.75 \times 10^{7}$ & $1 \times 10^{6}$ & $1.75 \times 10^{\circ}$ & $1 \times 10^{7}$ & $287 \times 10^{4}$ & $1.11 \times 10^{5}$ & $1.94 \times 10^{\prime}$ & $9.91 \times 10^{5}$ & $1.74 \times 10^{8}$ & $1.00 \times 10^{7}$ & $287 \times 10^{4}$ \\
\hline 5 & $5 \times 10^{5}$ & $8.76 \times 10^{7}$ & $5 \times 10^{5}$ & $8.76 \times 10^{7}$ & $5 \times 10^{6}$ & $1.44 \times 10^{4}$ & $5.12 \times 10^{5}$ & $8.97 \times 10^{7}$ & $4.97 \times 10^{5}$ & $8.70 \times 10^{7}$ & $5.20 \times 10^{6}$ & $1.49 \times 10^{3}$ \\
\hline 6 & $1 \times 10^{6}$ & $1.75 \times 10^{8}$ & $1 \times 10^{5}$ & $1.75 \times 10^{7}$ & $1 \times 10^{6}$ & $2.87 \times 10^{3}$ & $9.92 \times 10^{5}$ & $1.74 \times 10^{8}$ & $1.13 \times 10^{5}$ & $1.98 \times 10^{7}$ & $1.27 \times 10^{6}$ & $3.65 \times 10^{3}$ \\
\hline 7 & $1 \times 10^{6}$ & $1.75 \times 10^{8}$ & $1 \times 10^{5}$ & $1.75 \times 10^{7}$ & $1 \times 10^{7}$ & $287 \times 10^{4}$ & $1.01 \times 10^{6}$ & $1.77 \times 10^{8}$ & $1.11 \times 10^{6}$ & $1.94 \times 10^{8}$ & $9.91 \times 10^{6}$ & $284 \times 10^{4}$ \\
\hline 8 & $1 \times 10^{6}$ & $1.75 \times 10^{2}$ & $1 \times 10^{6}$ & $1.75 \times 10^{8}$ & $1 \times 10^{6}$ & $2.87 \times 10^{3}$ & $1.00 \times 10^{6}$ & $1.75 \times 10^{8}$ & $1.01 \times 10^{6}$ & $1.77 \times 10^{8}$ & $1.28 \times 10^{6}$ & $3.67 \times 10^{3}$ \\
\hline 9 & $1 \times 10^{6}$ & $1.75 \times 10^{8}$ & $1 \times 10^{6}$ & $1.75 \times 10^{8}$ & $1 \times 10^{7}$ & $2.87 \times 10^{4}$ & $1.01 \times 10^{6}$ & $1.77 \times 10^{8}$ & $1.02 \times 10^{6}$ & $1.79 \times 10^{2}$ & $9.96 \times 10^{6}$ & $2.86 \times 10^{4}$ \\
\hline
\end{tabular}


Figure 3.7 shows also the effect of the training set member spacing on the accuracy of the model parameter estimates. As the number of members in the training set increased, the spacing between members decreased because the range of spring rates used to form the training sets remained constant. As the training set member spacing decreased, the range over which the neural network must interpolate to estimate model parameters also decreases, thus accounting for the improvement in the accuracy of the model parameter estimates as the number of members in the training set is increased.

The results clearly show that the selection of eigenvalues and eigenvectors to form the training sets was appropriate. The calculation of these quantities is relatively simple and computationally efficient, especially when compared to extracting similar information from a calculated time response. Finally, we showed also that equivalent information can be extracted from a frequency spectrum and used to obtain an estimate of the model parameters. Thus, taken together, these results show that the neural network can be trained to solve the inverse problem in which the model spring rates are determined from spectral features such as resonance frequencies and mode shape components. Because these same spectral features can be extracted from measured signals, we anticipated that similar results would be obtained from the hardware demonstration. 


\section{PHASE II: APPLYING THE DIAGNOSTIC METHOD TO MEASURED DATA}

The bench-top test unit and the data acquisition system are described in this chapter. This equipment was used during the Phase II portion of the investigation to demonstrate the use of the diagnostic method on a relatively simple mechanical system. In addition to demonstrating the method, the Phase II work provides an indication of the effects of modeling and measurement errors on the accuracy of the model parameter estimates. Appendix $\mathrm{C}$ contains the detailed drawings of the bench-top test unit.

\subsection{THE BENCH-TOP TEST UNIT}

A description of the bench-top test unit and the data acquisition system are given in this section.

\subsubsection{Description of the Bench-Top Test Unit}

The bench-top test unit consists of three main structural members: the frame base, the top beam, and the test beam (Fig. 4.1). A motor-driven assembly consisting of an electric motor, coupling, shaft, and bearings is mounted on the test beam. The motor-driven assembly corresponds to the motor, coupling, and pump of the mathematical model. The test beam corresponds to the base plate of the mathematical model. The structural members of the bench-top test unit were designed to deflect only in the vertical plane. This constraint is imposed to force consistency between the deflections of the bench-top test unit and the calculated deflections of the mathematical model. The main purpose of the frame base and the top beam is to constrain the motion of the test beam. No motion constraint was applied to the motor/coupling/shaft assembly, because the motor was not used and the motor/coupling/shaft was excited only in the vertical direction during this investigation.

The frame base is constructed of 4-in. $(10.16-\mathrm{cm}) \times 5.4$ steel channel, $72 \mathrm{in} .(1.8 \mathrm{~m})$ in length. At each end of the base are two horizontal stabilizing members and an 18-in. $(46-\mathrm{cm})$ vertical upright, each constructed of 4 -in. $(10.16-\mathrm{cm}) \times 5.4$ steel channel and welded to the horizontal base. Each upright has two 0.390-in.- (0.99-cm-) wide vertical slots, 7.12 in. $(18.1 \mathrm{~cm})$ in length, to provide an adjustable attachment for the top beam. The frame base is placed on an 0.125 -in. $-(0.32-\mathrm{cm}-)$ thick neoprene rubber mat to provide some isolation from the bench top. The frame base is bolted to the bench top through the horizontal stabilizing members. The top beam is constructed of 4-in. $(10.16-\mathrm{cm}) \times 5.4$ steel channel and is bolted to the uprights at each end.

The test beam is constructed of $7-\mathrm{in} .(17.8-\mathrm{cm}) \times 9.80$ steel channel and is constrained to move only in the vertical plane by four rollers, two on each end of the beam. Theses rollers contact the faced outside surface of each upright and also act to prevent twisting of the test beam. The test beam is mounted to the base and to the top beam by four Firestone Model 1M1A air springs that correspond to the mounting springs $K_{m n}$ and $K_{m p}$. The use of air springs results in a convenient way to provide a known mounting spring rate that can be easily adjusted.

The dependence of the spring rate on air pressure of the $1 \mathrm{M} 1 \mathrm{~A}$ air springs at the design height of $2.5 \mathrm{in} .(6.35 \mathrm{~cm})$ is shown in Fig. 4.2. These spring rates may be calculated by using 


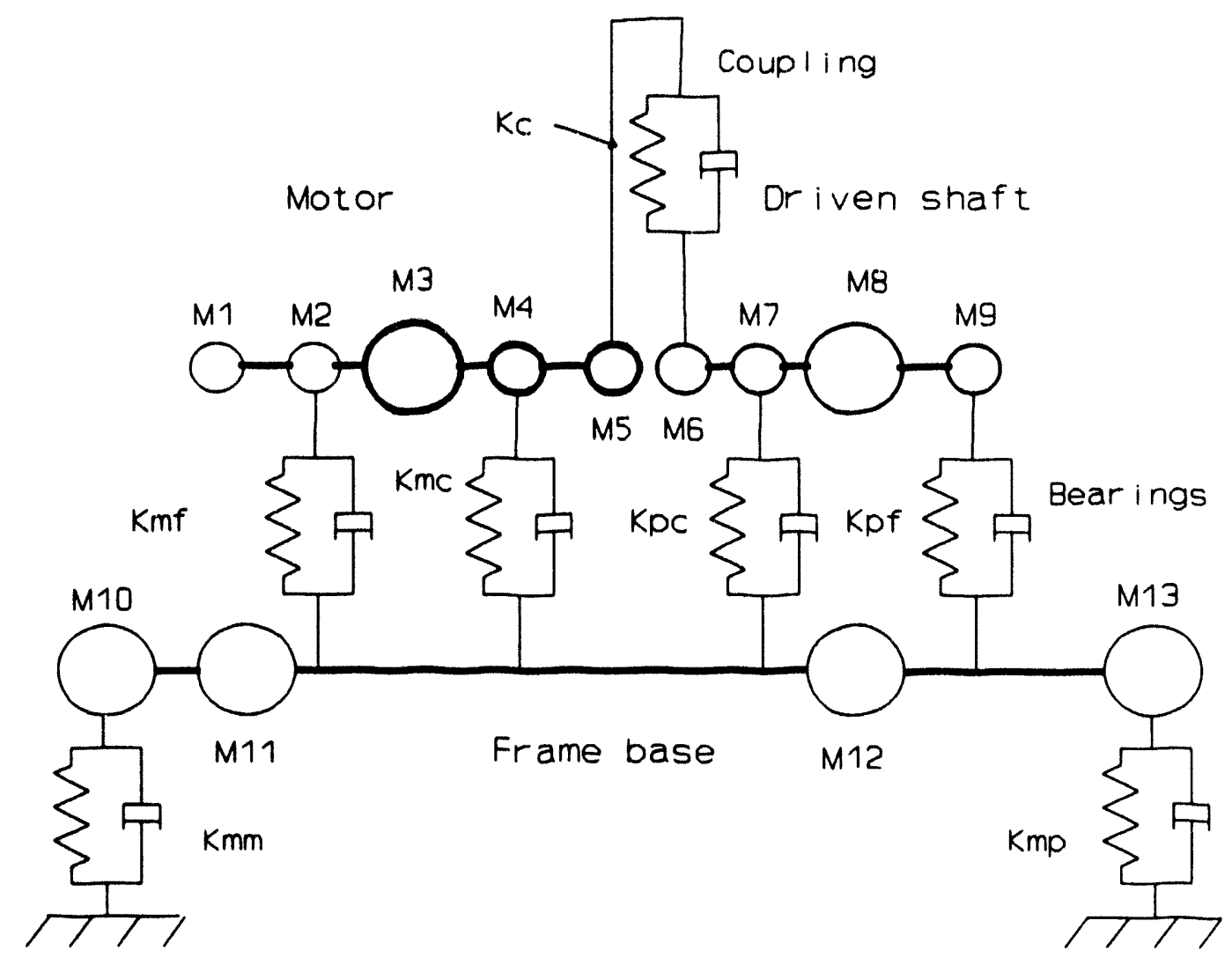

Fig. 4.1. The three-beam model of the bench-top test unit.

$K=\left(P_{s}+1.01 \times 10^{5}\right)\left[A_{c}\left(\frac{V_{1}}{V_{c}}\right)^{1.38}-A_{c}\left(\frac{V_{1}}{V_{c}}\right)^{1.38}\right]-1.01 \times 10^{5}\left(A_{c}-A_{c}\right)$

where

$K=$ the vertical spring rate in $\mathrm{N} / \mathrm{m}$,

$P_{g}=$ the gauge pressure at the design height in $\mathrm{Pa}$,

$A_{c}=$ the effective area at $0.5 \mathrm{in}$. below the design height in $\mathrm{m}^{2}$,

$A_{c}=$ the effective area at $0.5 \mathrm{in}$. above the design height in $\mathrm{m}^{2}$,

$V_{1}=$ the internal volume at the design height in $\mathrm{m}^{3}$,

$V_{c}=$ the internal volume at $0.5 \mathrm{in}$. below the design height in $\mathrm{m}^{3}$,

$V_{c}=$ the internal volume at $0.5 \mathrm{in}$. above the design height in $\mathrm{m}^{3}$ (reference 21). 
The nearly linearly relationship between the spring rate and the gauge pressure of the Firestone 1M1A air spring shown in Fig. 4.2 can be approximated by using

$$
K \approx 0.75 P_{8}+8.75 \times 10^{3}
$$

Because the bottom air spring must support the weight of the test beam, the top air springs require less pressure $(\sim 10 \mathrm{PSI})\left(6.9 \times 10^{4} \mathrm{~Pa}\right)$ than the bottom air springs to keep the test beam centered between the top and bottom of the frame. Each top air spring is individually controlled with an Norgren model R46-200-RNLA in-line pressure regulator. Individual pressure gauges are used to monitor the pressure in each air spring.

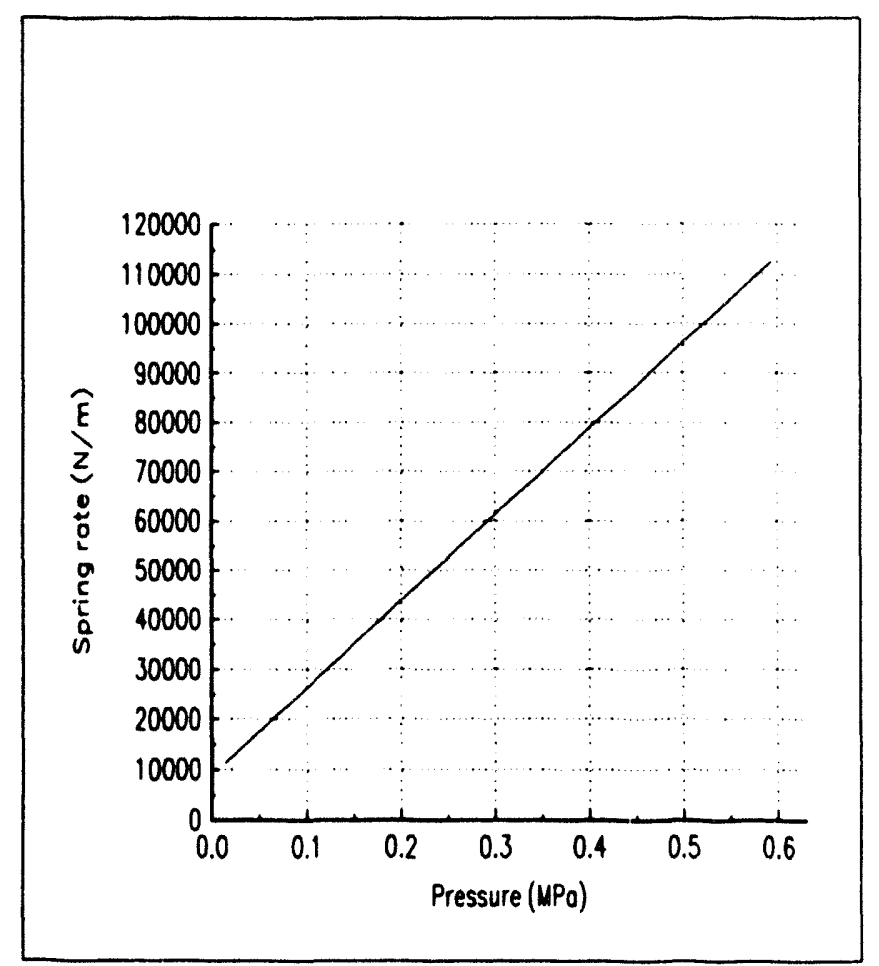

Fig 4.2. Effect of internal pressure on the spring rate of the Firestone 1M1A airspring.
The 0.25-in.- (0.64-cm-) diam motor shaft of the Dayton model 2M037A electric motor is attached to the 0.5 -in.- $(1.27-\mathrm{cm}$-) diam driven steel shaft through a Lovejoy Model L050 rubber spider coupling. The driven shaft is supported at each end by a Browning Model 16X232CVPS-108 bearing block assembly. The ball bearings have a nominal inner race outside diameter of 0.88 in. $(2.2 \mathrm{~cm})$, a nominal outer race inside diameter of 1.388 in. $(3.525 \mathrm{~cm})$, and contain 9 rolling elements. Relative motion of the rolling elements is prevented by a roller cage. The entire assembly is centered on the test beam.

Three Endevco Model 2233E accelerometers and one eddycurrent proximity probe are used to measure the vibration of the bench-top test unit. The signal from each accelerometer is amplified by an Endevco Model 2721A charge amplifier before

being supplied to the data-acquisition system. Each end of the test beam has one accelerometer stud-mounted on the center line next to the air spring to measure the absolute movement of each end of the test beam. The proximity probe measures the relative movement between the top beam and the center of the driven shaft, and an accelerometer is mounted on the top beam next to the proximity probe. The accelerometer and proximity probe measurements can be used to obtain the absolute movement of the center of the driven shaft. 


\subsubsection{Description of the Data-Acquisition System}

The data-acquisition system consisted of an IBM-compatible 486 PC computer equipped with a 16-bit, multiple-channel digital data-acquisition board (AT-MIO-16X from National Instruments Corporation). The LabVIEW data-acquisition package, also from National Instruments Corporation, was used as the software driver. ${ }^{n}$ All data were lowpass-filtered by using a Rockland 852 active-filter prior to digitization.

\subsection{MATHEMATICAL MODEL OF THE BENCH-TOP TEST UNIT}

The three-beam pump model described in Sect. 2.1 was modified to simulate the vibration of the bench-top test unit. The modifications were as follows:

1. Four mass points were used to represent the mass distribution of the test beam. To properly describe the uniform mass distribution of the test beam, the mass points were located at each end of the beam and at one third of the test beam length from each end.

2. The individual beam-bending stiffnesses between each mass point were eliminated because the test beam is uniformly stiff along its length.

3. The mounting springs were moved to the end of the test beam to more closely model the attachment of the test beam to the frame base.

A diagram of the bench-top test unit model is shown in Fig. 4.1. Table 4.1 lists the components represented by each lumped parameter in the model and lists also the parameter values that describe the bench-top test unit. The mass matrix, the stiffness matrix, and the undamped eigenvalues and eigenvectors were obtained by performing the calculations described in Sect. 2.1.1.

The bearing radial spring rates were calculated by using

$$
K_{r}=0.0325 \times 10^{6} \sqrt[3]{D F, Z^{2} \cos ^{5}(\alpha)}
$$

where

$D=$ rolling element diameter in in.,

$F_{r}=$ the applied radial force in $\mathrm{lb}_{\mathrm{p}}$,

$Z=$ the number of rolling elements,

$\alpha=$ the contact angle in radians. ${ }^{23}$

By using Eq. (4.3), assuming a contact angle of 0 radians, and assuming an equal distribution of weight on each bearing results in calculated radial spring rates of $7.7 \times 10^{4} \mathrm{lb} / \mathrm{in}$. $\left(1.35 \times 10^{7} \mathrm{~N} / \mathrm{m}\right)$ for the bearing blocks and $4.5 \times 10^{4} \mathrm{lb} /$ in. $\left(7.88 \times 10^{6} \mathrm{~N} / \mathrm{m}\right)$ for the motor bearings. The initial values of the beam-bending stiffnesses were calculated by taking the product of the modulus of elasticity and the area moment of inertia of each beam. 
Table 4.1. Bench-top test unit model lumped parameters

\begin{tabular}{|c|c|}
\hline Model parameter & System component \\
\hline $\mathbf{M}_{1}$ & Motor fan and part of motor shaft \\
\hline $\mathbf{M}_{2}$ & Outboard motor bearing mass, part of motor shaft \\
\hline $\mathbf{M}_{\mathbf{3}}$ & Motor armature mass, part of motor shaft \\
\hline $\mathbf{M}_{4}$ & Inboard motor bearing mass, part of motor shaft \\
\hline $\mathbf{M}_{\mathbf{s}}$ & Motor half of coupling, part of motor shaft mass \\
\hline M & Driven shaft half of coupling, part of driven shaft \\
\hline $\mathbf{M}_{7}$ & Inboard bearing block bearing mass, part of driven shaft mass \\
\hline $\mathbf{M}_{\mathbf{8}}$ & Part of driven shaft mass \\
\hline M, & Outboard bearing block bearing mass, part of driven shaft \\
\hline $\mathbf{M}_{10}$ & Quarter of frame base mass \\
\hline $\mathbf{M}_{11}$ & Half of frame base mass, motor case mass \\
\hline $\mathbf{M}_{12}$ & Half of frame base mass \\
\hline $\mathbf{M}_{13}$ & Quarter of frame base mass \\
\hline$K_{m}, K_{\infty x}$ & Motor bearing spring rate \\
\hline $\mathbf{K}_{p e,} \mathbf{K}_{p}$ & Bearing block bearing spring rate \\
\hline$K_{m}, K_{m}$ & Spring rate of the air springs \\
\hline $\mathrm{EL}_{\mathbf{m}}$ & Beam stiffness of motor shaft \\
\hline $\mathrm{EI}_{\mathrm{p}}$ & Beam stiffness of driven shaft \\
\hline EI & Beam stiffiness of frame base \\
\hline $\mathrm{K}_{\mathrm{c}}$ & Coupling spring rate \\
\hline
\end{tabular}

\section{Model Tuning}

Before the mathematical model of the bench-top test unit could be used to form a neural network training set, the nominal model parameter values had to be adjusted to obtain satisfactory agreement between the calculated and measured resonance frequencies and modes. The mass-point mass values and locations were held fixed during model tuning because these values are easily and accurately calculated. The bearing spring rates were also held constant during model tuning because these values had no effect on the low-frequency modes used as neural network input.

The beam-bending stiffnesses $E I, E I_{m}$, and $E I_{p}$ were adjusted by trial and error during model tuning. Neglecting to include the rotational inertia at the mass points and the trunnion spring rate of the bearings results in a model that underpredicts the stiffness of 
the bench-top test unit. Thus, the calculated resonance frequencies of the flexural modes of the test beam, motor shaft, and driven shaft will be lower than the measured resonance frequencies of theses modes. The values of $E I, E I_{m}$, and $E J_{p}$ were adjusted from their initial values to tuned values, in which good agreement tetween the resonance frequencies of the flexural modes was obtained. Table 4.2 lists the measured resonance frequencies as well as the resonance frequencies calculated by using both the initial and tuned values of $E I, E I_{m}, E I_{p}, K_{m m}$, and $K_{m p}$.

Table 4.2. Resonant frequencies of the bench-top test unit model

\begin{tabular}{lccc}
\hline \multicolumn{1}{c}{ Mode } & $\begin{array}{c}\text { Initial } \\
(\mathrm{Hz})\end{array}$ & $\begin{array}{c}\text { Tuned } \\
(\mathrm{Hz})\end{array}$ & $\begin{array}{c}\text { Measured } \\
(\mathrm{Hz})\end{array}$ \\
\hline First rigid body & 17.6 & 18.3 & 18.5 \\
Second rigid body & 24.9 & 25.6 & 26.0 \\
First test beam flexural & 86.2 & 110.4 & 110.0 \\
First driven shaft flexural & 128.1 & 168.3 & 168.0 \\
Second test beam flexural & 197.2 & 251.7 & 267.0 \\
First motor shaft flexural & 496.0 & 521.7 & 522.0 \\
\hline
\end{tabular}

\subsection{Measured Data}

The data were collected for 36 combinations of air spring pressures ranging from 20 to 70 psig $\left(1.38 \times 10^{5}\right.$ to $\left.4.83 \times 10^{5} \mathrm{~Pa}\right)$ in 10-psig $\left(6.90 \times 10^{4} \mathrm{~Pa}\right)$ increments. For each combination, nine data sets were taken by using a sampling rate of 1000 samples/s and a 1024-sample blocksize. Vibrational modes were excited by impacting the beam on the left and right sides and impacting the motor shaft. Each data set was then Fourier transformed, and the autopower spectral density calculated. The resulting spectrum for each pressure combination is the average of the nine sets of spectra obtained. Three resonance frequencies were used for diagnostic analysis (see Sect. 4.4). Included were resonance frequencies for the first and second rigid-body modes and the first driven-shaft flexural mode. Appendix D shows these frequencies and the amplitudes of the three measured mode shape components for each combination of air spring pressure.

\subsection{FORMATION OF THE TRAINING SETS AND TRAINING OF THE NEURAL NETWORK}

The range of values of $K_{m m}, K_{m p}$, and $E I_{p}$ contained in the training set were selected so the calculated resonance frequencies of the three modes listed in Appendix D spanned the range of measured values. Both $K_{m m}$ and $K_{m p}$ ranged from 350 to $1200 \mathrm{lb} /$ in. 


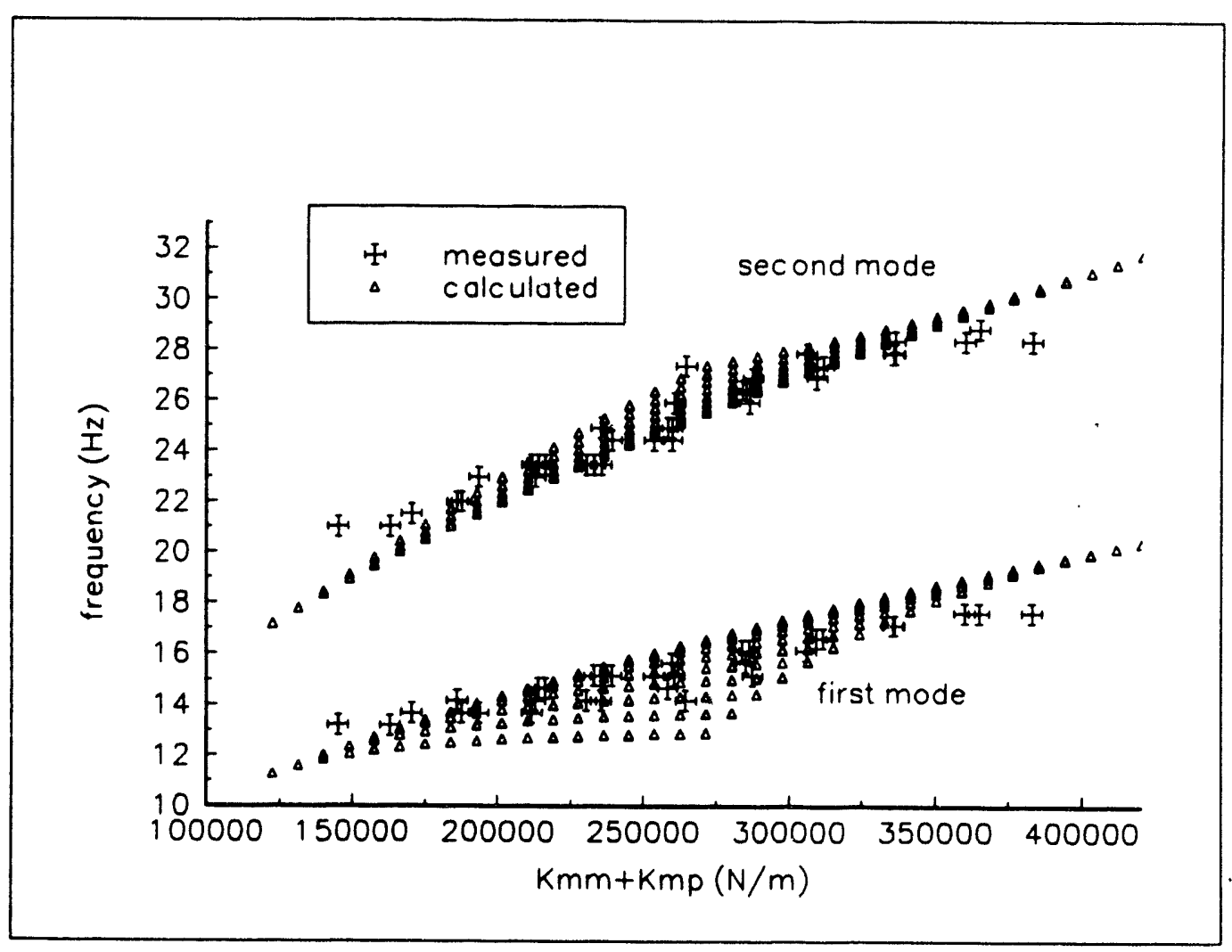

Fig. 4.3. Comparison of the measured and calculated values of resonant frequency.

$\left(6.13 \times 10^{4}\right.$ to $\left.2.10 \times 10^{5} \mathrm{~N} / \mathrm{m}\right)$ in 50-1b/in. $\left(8.76 \times 10^{3} \mathrm{~N} / \mathrm{m}\right)$ increments, and $E I_{p}$ ranged from $1.5 \times 10^{5}$ to $1.8 \times 10^{5} \mathrm{lb}_{\mathrm{f}} \cdot$ in. $^{2}\left(430\right.$ to $\left.517 \mathrm{~N} \cdot \mathrm{m}^{2}\right)$ in $1.0 \times 10^{4} \mathrm{lb}_{\mathrm{f}} \cdot$ in. $^{2}\left(29-\mathrm{N}^{\prime} \cdot \mathrm{m}^{2}\right)$ increments. Figure 4.3 shows a comparison of the measured and calculated frequencies of the first two rigid-body modes, calculated by using the values of $K_{m m}$ and $K_{m p}$ in the training set. These results show good, but not perfect, agreement between the measured and calculated resonance frequencies.

The combination of spring rates used to form the training set resulted in a 972-member training set. The neural network input consisted of the resonance frequency and the normalized mode shape components of each end of the test beam and of the center of the driven shaft (i.e., mass points 10,13 , and 8) for the first two rigid-body modes and for the first flexural mode of the driven shaft. The neural network output was the values of $K_{m m}, K_{m p}$, and $E I_{p}$.

The neural network for this case required 12 inputs (three frequencies and three mode shape components per mode). Therefore, for consistency with the previous networks, a single hidden layer of approximately one-half the number of input nodes (i.e., 6) was used. Also, as before, the hyperbolic tangent transfer function was used, and the cumulative delta learning rule was employed with an epoch of 100 . Best results were obtained for a training total of 70,000 iterations. Figure 4.4 shows results for the trained network tested with the training set for the two spring rates $K_{m m}$ and $K_{m p}$. Average errors 


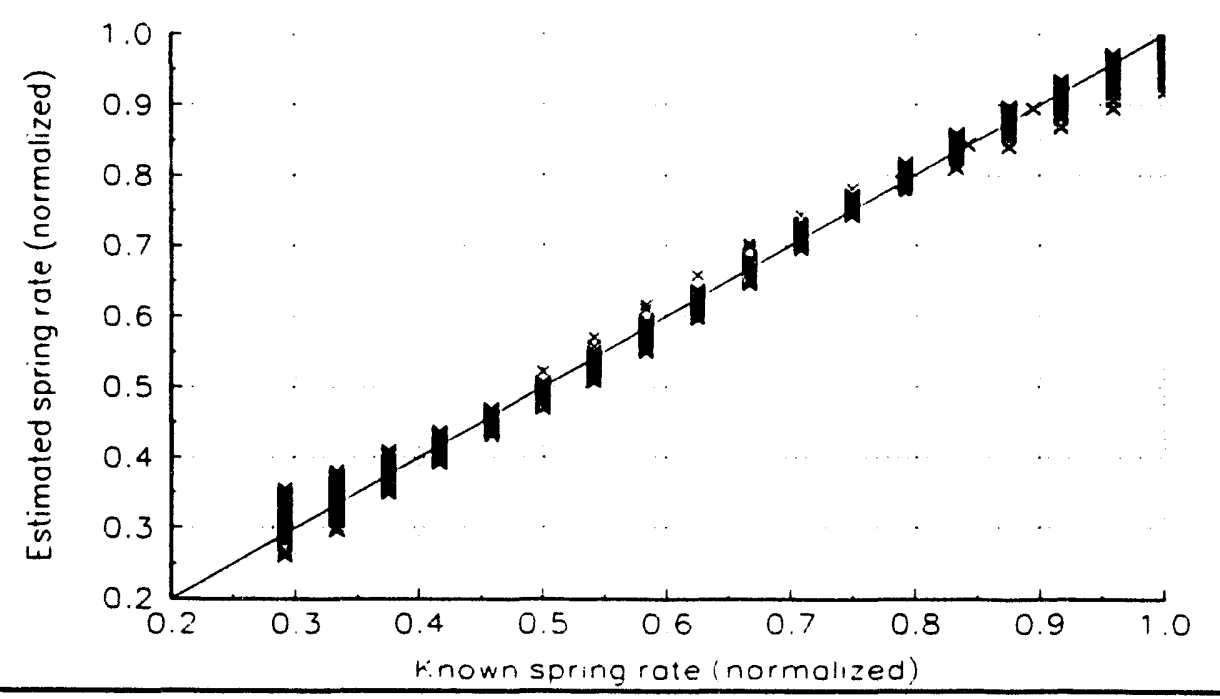

Fig. 4.4. Comparison of the known and estimated spring rate values for spring rates contained in the training set.

in this case were $2.04 \%$ for $K_{m m}$ and $2.34 \%$ for $K_{m p}$, with standard deviations of 1.41 and $1.71 \%$ respectively. These results are approximately the same as those obtained during the Phase I analysis. Note that results obtained for the middle of the output range are somewhat better than those for the high and low extremes.

\subsection{RESULTS OBTAINED FROM APPLYING THE DIAGNOSTIC METHOD TO MEASURED DATA}

The 36 sets of experimental results discussed in Sect. 4.2 .2 were tested by using the network trained from the tuned model of the experimental bench-top unit. Figure 4.5 shows results for the spring rates $K_{m m}$ and $K_{m p}$. Average errors are $5.91 \%$ for $K_{m m}$ and $10.7 \%$ for $K_{m p}$, with standard deviations of 5.30 and $10.1 \%$ respectively. Note that the $K_{m p}$ values are well grouped but fall beneath the unit line shown in Fig. 4.5, that is, the actual value of the spring rate tends to be larger than the estimated value. This difference is what accounts for the larger error in $K_{m p}$. Otherwise, results for the two cases are considered similar. It should be remembered that several sources of uncertainty are combined in Fig. 4.5. Included are the uncertainties in the mathematical model, uncertainties in the neural network interpolation as discussed previously, and uncertainties in the data acquisition. Data-acquisition errors include difficulty in obtaining a precise pressure reading and some question as to the accuracy of the pressure-to-spring rate conversion equation. This latter concern may explain the increased error in $K_{m p}$. With these considerations in mind, results in the 5 to $10 \%$ range are quite good. 


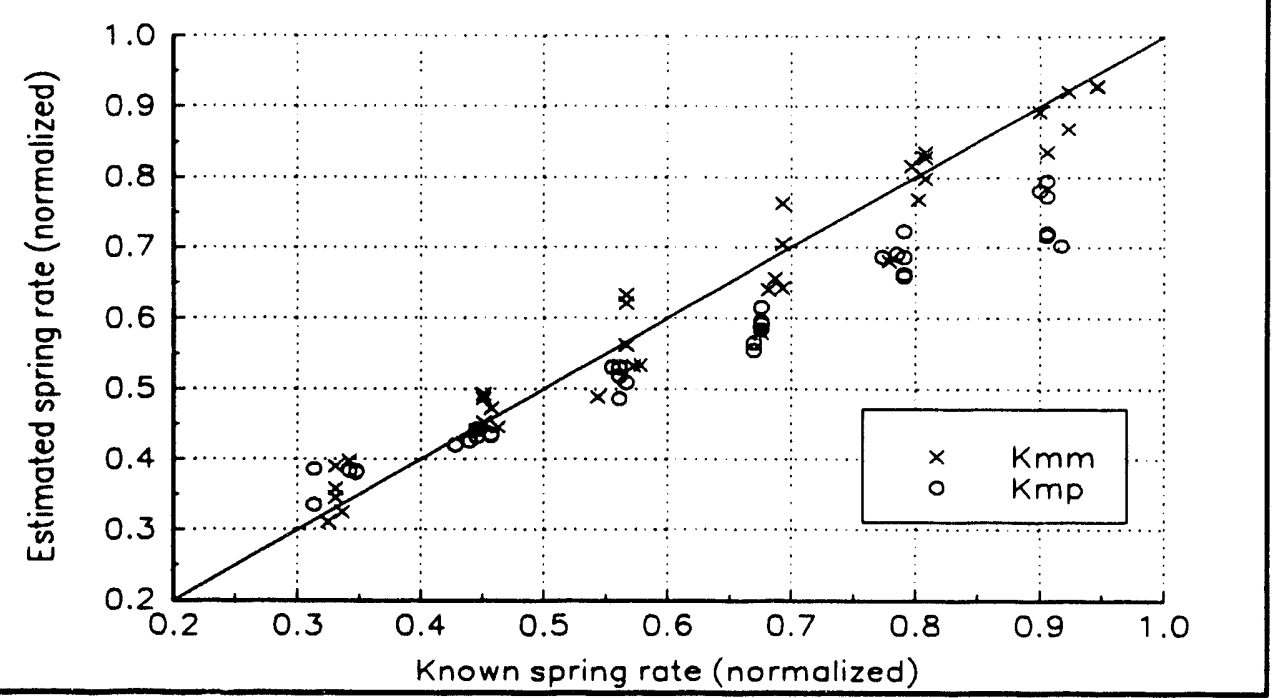

Fig. 4.5. Comparison of the known and estimated spring rate values made by using measured data.

Figure 4.6 shows a parametric display of the variation in $K_{m m}$, with $K_{m p}$ held approximately constant. Figure 4.7 shows a similar display for $K_{m p}$, with $K_{m m}$ approximately constant. In both figures, three plots are shown with the constant parameter at $376 \mathrm{lb} / \mathrm{in}$., $752 \mathrm{lb} /$ in., or $1066 \mathrm{lb} /$ in. $\left(6.59 \times 10^{4} \mathrm{~N} / \mathrm{m}, 1.32 \times 10^{5} \mathrm{~N} / \mathrm{m}, 1.87 \times 10^{5} \mathrm{~N} / \mathrm{m}\right)$. These values correspond to pressure values of 20,40 , and $70 \mathrm{psig}\left(1.379 \times 10^{5} \mathrm{~Pa}\right.$, $\left.2.76 \times 10^{5} \mathrm{~Pa}, 4.83 \times 10^{5} \mathrm{~Pa}\right)$. These plots show that trends in one of the spring rates can easily be detected. That is, for example, a $10 \%$ change in one of the parameters would result in about a $10 \%$ change in the estimated value regardless of the initial agreement between the estimated and actual values.

\subsection{DISCUSSION OF RESULTS}

The demonstration clearly shows the ability of the diagnostic method to estimate the mounting spring rate values to within 5 to $10 \%$. Thus, it can be concluded from this demonstration that for mechanical systems in which the relationship between the measured output (neural network input) and the monitored system parameters (neural network output) is single valued and which show significant changes in the measured output for significant changes in the monitored system parameters, a successful application of the monitoring method to detect, locate, and estimate the magnitude of structural changes can be expected. 


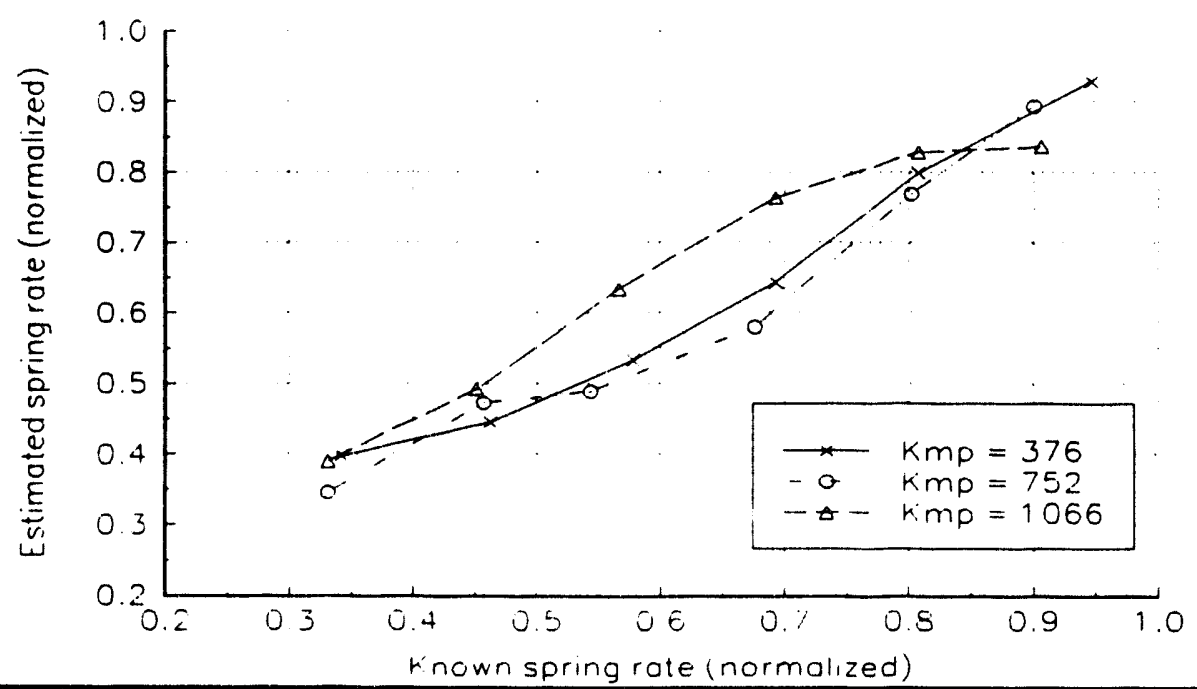
fixed.

Fig. 4.6. Estimated value of $K_{m m}$ calculated while holding the value of $K_{m p}$

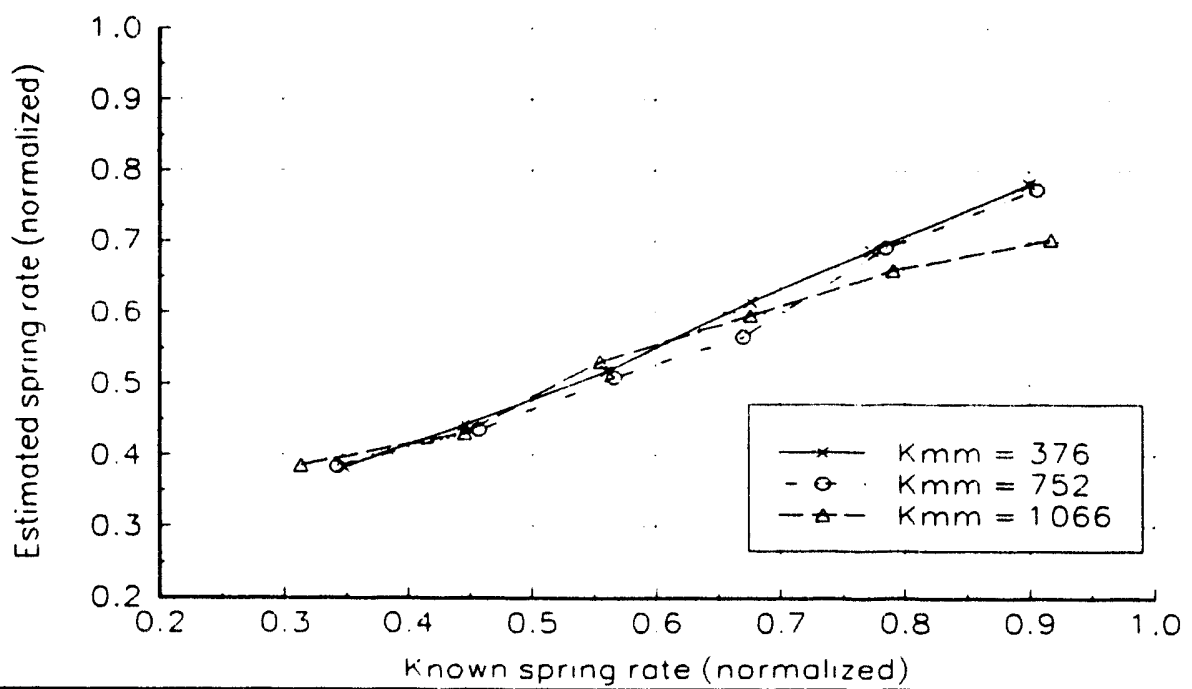
fixed.

Fig. 4.7. Estimated value of $K_{m p}$ calculated while holding the value of $K_{m m}$ 
The three main sources of error in the model parameter estimates are modeling errors, neural network errors, and measurement errors. The results from the computer simulation indicate that the neural network results are on the order of 2 to $3 \%$. The model error, as indicated by the comparison of the measured and calculated values shown in Fig. 4.3 , is believed to be $\sim 5 \%$. The measurement errors are also believed to be on the order of $5 \%$. These errors are attributed to leaky pressure regulators that allowed the air spring pressure to vary during each measurement, a sticking pressure gauge on one air spring, and the unit-to-unit variability that would cause errors in the air spring

pressure-to-spring rate conversion. Considering the task performed by the neural network, it is expected that both modeling and measurement errors would have a significant effect on the accuracy of the method's parameter estimation. Either type of error would result in a mismatch between the measured quantities and the training set input values, causing poor parameter value estimation. Thus, it appears that both the modeling and the measurements need to be carefully performed to achieve optimum parameter estimates with the diagnostic method. 


\section{SUMMARY AND CONCLUSIONS}

Computer simulation results and a demonstration using a bench-top test unit were used to determine that the diagnostic method using frequency spectra to estimate structural parameters can be successfully applied to detect and locate structural changes in a mechanical system. In particular, it was shown that a neural network, trained by using eigenvalues and eigenvector components calculated by a mathematical model, can be used to estimate the structural condition of the mechanical system by using measurements of resonance frequencies and mode shape components extracted from vibration spectra. We concluded that the diagnostic method should be capable of being successfully applied to monitor the structural condition of a mechanical system with the following characteristics:

1. The relationship between the measured parameters (neural network input) and the monitored parameters (neural network output) must be single valued if the neural network is to train properly.

2. Changes in the monitored parameters must have a significant effect on the values of the measurable parameters.

A discussion of specific results and conclusions based on the computer simulation results and on the demonstration using the bench-top test unit are presented next.

\subsection{RESULTS AND CONCLUSIONS BASED ON THE COMPUTER SIMULATION RESULTS}

The computer simulation addressed the following questions:

1. What effect does the training set composition and size have on the accuracy of the model parameter estimates made by the neural network?

2. Is the formation of the training set or the neural network training so computationally intensive that the diagnostic method is impractical?

3. Are eigenvalues and eigenvector components a practical choice for forming the neural network training set, and is the information contained in these values sufficient for the diagnostic method to accurately predict model parameters?

4. Can information equivalent to the eigenvalues and eigenvector components used to form the training set be easily extracted from vibration spectra?

5. Can the trained neural network solve the "inverse proble.n," that is, can the neural network be used to accurately estimate the model parameters that correspond to a given set of eigenvalues/eigenvectors (resonance frequencies and mode shapes)? 
Thus, most of the important considerations concerning the applicability of the monitoring method are addressed by the computer simulation portion of the investigation.

The simulation results show that the accuracy of the neural network parameter estimation depends heavily on the composition and the "spacing" of the members in the training set. If the training set composition is such that the relationship between the neural network input and output is not single valued, the resulting model parameter estimation is poor. An example of this behavior is the relatively high error associated with training sets 1, 2, and 3 (Fig. 3.7). Because these training sets contained only resonance frequencies as input, in some cases more than one combination of spring rates resulted in nearly identical resonance frequency values. Including mode shape components in training sets 4 through 9 avoids this problem, producing better parameter estimates, as shown by the lower error values obtained by using these training sets.

Figure 3.7 shows also that the spacing between members in the training set affects the accuracy of the estimated spring rates. As the number of training set members increases, the spacing between adjacent members decreases because the parameter range remains constant for all training sets. Thus, the interpolation between training set members performed by the neural network when estimating spring rate values occurs over a smaller interval as the training set member spacing decreases, resulting in more accurate spring rate estimates.

The amount of computation required to form the training set and train the neural network was not prohibitively large in the applications of the diagnostic method used in this work. Although the applicability of this statement is obviously limited by the relatively simple models and the small number of parameters adjusted in this work, no reason is apparent to expect prohibitively large calculations for significantly larger models. Thus, this question remains open at this time but does not appear to pose a great threat to the practical application of the diagnostic method.

The results obtained from the ACSL-calculated spectra clearly show that, as expected, information equivalent to the calculated eigenvalues and eigenvector components can be obtained from vibration spectra. These results also show that using calculated eigenvalues and eigenvectors to form the neural network training set is an effective and computationally efficient choice. However, one drawback to using eigenvector components in the training set is that the number of required sensors equals the number of eigenvector components contained in the training set. Thus, it may be impossible in some applications to obtain all of the measurements required to produce the necessary eigenvector (mode-shape) components.

Finally, the results from the computer simulation, taken together, show that the trained neural network can accurately (to within $3 \%$ ) solve the inverse problem of determining model parameters from the resonance frequencies and mode-shape components. The results of the computer simulation indicate that the diagnostic method should be applicable to real mechanical systems that have a single-valued relationship between the neural network input and output and that have monitored parameters have a significant effect on the measurable quantities.

\subsection{RESULTS AND CONCLUSIONS BASED ON THE APPLICATION OF THE DIAGNOSTIC METHOD TO THE BENCH-TOP TEST UNIT}

The application of the diagnostic method to the bench-top test unit was intended primarily as a demonstration of the method on a simple mechanical system. In addition to 
demonstrating the method, an indication of the effect of modeling and measurement errors on the method's accuracy was obtained.

The demonstration clearly shows the capability of the diagnostic method to estimate (to within 5 to $10 \%$ ) values of the mounting spring rates. Thus, we concluded from the demonstration that the diagnostic method can be used to detect, locate, and estimate the magnitude of structural changes in mechanical systems that have a single-valued relationship between neural network input and output and that have monitored parameters that significantly affect the measured parameters.

The effect of modeling and measurement errors on the method's accuracy, although not specifically investigated, are indicated by the results. The three main error sources are modeling errors, neural network errors, and measurement errors. From the results shown in Chap. 3 and in Fig. 4.4, the neural network errors are known to be on the order of $2 \%$. The model error, indicated by the comparison of calculated and measured values shown in Fig. 4.3, is estimated to be on the order of $5 \%$. The measurement errors, although not quantified, are believed to be relatively large, on the order of 5\%. This error was due to difficulty with the pressure regulators (which continuously bled air, changing the air spring pressure), stickiness in one of the pressure gauges, and the unavoidable unit-to-unit variability that introduced errors into the pressure-to-spring rate equation. Both modeling and measurement errors will cause a mismatch between the measurements and the neural network input values contained in the training set, resulting in poor system parameter estimates. Thus, both the system modeling and the measurements must be performed with a considerable level of care if the method is to provide accurate parameter estimates.

Note that this sensitivity to modeling error does not necessarily mean that complicated mathematical models are always needed. The required model complexity will depend on the dynamic characteristics that need to be measured to detect changes in the monitored system parameters. If parameters such as the mounting spring rates need to be monitored, as was done in this work, the rigid-body modes supply all the information needed to detect and locate changes in these spring rates. These modes can be accurately modeled by using a relatively simple model, as shown by the results in Chap. 4 . If, on the other hand, system parameters such as shaft flexural modes that affect higher modes need to be monitored, a more complex model would be needed.

Finally, it should be pointed out that, although the diagnostic method has been presented only in connection with vibration signature interpretation, this is really a specific application of a more general methodology. The general methodology can be summarized in three steps: (1) form a training set from mathematical simulation results, (2) train a neural network to estimate model input parameters from model output values, and (3) use the neural network to monitor the system (simulation model) parameters over time, thus detecting and identifying the source of changes in measured values. This methodology has a range of application beyond vibration signature analysis. This technique should be applicable to monitor parameters in any system or process that satisfies the requirements that the relationship between the measured output and the monitored parameters be single-valued, that shows sufficient sensitivity to the parameters being monitored, and that can be accurately modeled. Thus, the results presented in this report, in addition to showing that the diagnostic method can be applied to detect and locate the source of changes in vibration signatures, also serves as a successful demonstration of the more general methodology. 


\section{REFERENCES}

1. J. A. Thie, Power Reactor Noise, American Nuclear Society, LaGrange Park, Ill., 1981.

2. J. S. Mitchell, An Introduction to Machinery Analysis and Monitoring, PennWell Publishing, Tulsa, Okla., 1981.

3. F. J. Sweeney, Utility Guidelines for Reactor Noise Analysis, EPRI NP-4970, Electric Power Research Institute, Palo Alto, Calif., 1987.

4. B. Damiano and R. C. Kryter, Current Applications of Vibration Monitoring and Neutron Noise Analysis: Detection and Analysis of Structural Degradation of Reactor Vessel Internals from Operational Aging, NUREG/CR-5479, ORNL/TM-11398, Oak Ridge National Laboratory, 1990.

5. F. J. Sweeney and D. N. Fry, "Thermal Shield Support Degradation in Pressurized Water Reactors," Flow-Induced Vibration-1986, PVP.Vol. 104, American Society of Mechanical Engineers, New York, 1986.

6. B. T. Lubin, R. Longo, and T. Hammel, "Analysis of Internals Vibration Monitoring and Loose Part Monitoring System Data Related to the St. Lucie Thermal Shield Failure," Prog. Nucl. Energy 21, 117-26 (1987).

7. R. Sunder and D. Wach, "Reactor Diagnosis Using Vibration and Noise Analysis in PWRs," in Operational Safety of Nuclear Power Plants I, International Atomic Energy Agency, Vienna (1984).

8. C. Puyal et al., "Solution to Thimble Vibrations of French and Belgian Reactors Using Accelerometers and Neutron Noise," 19th Informal Meeting on Reactor Noise, Rome, June 4-6, 1986.

9. W. T. Thomson, Theory of Vibration with Applications, Prentice Hall, Englewood Cliffs, N.J., 1972.

10. Advanced Continuous Simulation Language Reference Manual, Ed. 10.0, Mitchell \& Gauthier Associates, Inc., Concord, Mass., 1991.

11. R. T. Wood, "A Neutron Noise Diagnostic Methodology for Pressurized Water Reactors," Ph.D. dissertation, The University of Tennessee, Knoxville, 1990.

12. R. T. Wood and R. B. Perez, "Modeling and Analysis of Neutron Noise from an Ex-Core Detector at a Pressurized Water Reactor," SMORN VI, A Symposium on Nuclear Reactor Surveillance and Diagnostics, Gatlinburg, Tenn., May 19-24, 1991.

13. D. O. Hebb, The Organization of Behavior, A Neuropsychological Theory, Wiley, New York, 1949. 
14. T. Kohonen, Self-Organization and Associative Memory, 3rd ed., Springer-Verlag, Berlin, 1989.

15. G. A. Carpenter and S. Grossberg, "A Massively Parallel Architecture for a Self-Organizing Neural Pattern Recognition Machine," Comput. Vision Graphics Image Process. 37 (1987).

16. R. O. Duda and P. E. Hart, Pattern Classification and Scene Analysis, Wiley, New York, 1973.

17. K. S. Narendra and K. Parthasarathy, "Identification and Control of Dynamical Systems Using Neural Networks," IEEE Trans. Neural Networks 1(1) (March 1990).

18. A. Lapedes and R. Farber, Nonlinear Signal Processing Using Neural Networks: Prediction and System Modeling, LA-UR-87-2662, Los Alamos National Laboratory, June 1987.

19. M. Smith, Neural Networks for Statistical Modeling, Van Nostrand Reinhold, New York, 1993.

20. NeuralWorks Professional II/PLUS and NeuralWorks Explorer Reference Guide, NeuralWare, Inc., Pittsburgh, 1991.

21. Firestone Airstroke Actuators, Airmount Isolators Engineering Manual and Design Guide, Firestone Industrial Products Company, Noblesville, Ind.

22. LabVIEW Users Manual, National Instruments Corp., Austin, Tex. 1993.

23. E. P. Gargiulo, “A Simple Way to Estimate Bearing Stiffness," Mach. Des. 107-10 (July 24, 1980). 
Appendix A

FREQUENCY SPECTRUM DECOMPOSIIION TECHNIQUE 
A model describing the frequency spectrum resulting from the vibration of a mechanical system was developed at Oak Ridge National Laboratory and successfully applied to detector noise data obtained from a nuclear power plant. ${ }^{1}$ This model characterizes peaks in the noise spectra in terms of frequency, width, amplitude, and skewness. As a result, a frequency spectrum can be decomposed into parameters representing the mechanical motion of system components.

The response of a detector to vibrations depends on the interaction of the vibratory motion with the detection process. For the derivation of the detector spectrum model, the vibration amplitudes of the system are convolved with window (or detector field of view) functions, which describe the physics of the detection process, to represent the detector response to each motion. Contributions from each resonance motion to the detector signal are summed to give the total detector response. An expression for the power spectral density (PSD) of the measured signal is formed by using the Fourier transform of the detector response. The detector PSD is given by

$$
\Phi_{\infty \omega}(\omega)=\sum_{\lambda}\left[\frac{\mu_{\lambda} A_{\lambda}+\left(\omega-\nu_{\lambda}\right) B_{\lambda}}{\mu_{\lambda}^{2}+\left(\omega-\nu_{\lambda}\right)^{2}}\right]+B G
$$

where $\omega$ is the mechanical vibration frequency, $\lambda$ is an index that varies over the frequencies of the mechanical vibrations, $A_{\lambda}$ is the pole strength or amplitude of the $\lambda$ th resonance, $B_{\lambda}$ is the asymmetry or skewness factor for the $\lambda$ th resonance, $\mu_{\lambda}$ is the damping coefficient for the $\lambda$ th resonance, $\nu_{\lambda}$ is the damped frequency of vibration for the $\lambda$ th resonance, and $B G$ is the background arising from the low-frequency feedback dynamics of the process. The pole strength describes the magnitude of the effect of the vibration on the detector response. The skewness factor represents the amount of interference in the detector response to one particular vibration mode resulting from competing perturbations introduced by other vibration modes.

The shapes given by the detector spectrum model peak at the resonance frequency for each $\lambda$ mode of motion. In cases where the "tails" or off-resonant response of modes at different frequencies do not contribute significantly to the amplitude of a modal resonance peak (i.e., light modal coupling exists), the resonance parameters of that mode can be determined by a single mode fit. However, in instances where the measured data near a peak are strongly influenced by off-resonant effects (i.e., heavy modal coupling exists), all the modal parameters must be identified simultaneously or the interference among the different vibrational modes must be determined.

For many measurements, PSDs exhibit heavy modal coupling, resulting in composite peaks composed of many closely spaced resonances seen by the detector. In some instances, initially isolated peaks may merge over time into what appears to be a single resonance peak, making it difficult to attribute changes to particular physical effects. This effect represents a major consideration in the development of the detector spectrum model and is described by the asymmetry factor in the PSD model. By including these terms in the PSD model, it is possible to characterize the coupling between modes of vibration, thereby separating the motions and allowing the resonance parameters for each peak to be extracted. The form of the detector spectrum model permits a quantitative investigation of the resonance structure of the PSD through identification of the parameters describing each peak. 
Other peaks not initially present may appear during the monitoring cycle. The effect of these submerged peaks can be accounted for by adding fitting peaks to represent them. Because it is important to compare fitted parameters from comparable models, the fitting algorithm can be configured to fit the model with a varying number of peaks during each measurement to provide a set of resonance parameters that can be used should unanticipated submerged peaks appear or visible peaks disappear.

The detector spectrum model has been implemented as user-supplied functions and derivative subroutines in a generalized least-squares fitting code. The background term can be formed from a low-frequency dynamic feedback model (if available) or can be user supplied. The background parameter included in the detector spectrum model fit represents the integral magnitude of the feedback dynamics contribution to the spectra. If no such information is available about the process dynamics, then the background can be addressed by fitting an additional, nonphysical peak to the low-frequency portion of the spectrum.

The values of the resonance parameters for each fit following the first can be used as initial parameter estimates for the subsequent fit. In this way, insight into the evolution of the spectra gained at each application of parameter estimation is available as an a priori input to the next fit. This insight can prove valuable in the cases where component vibrations become closely coupled during a monitoring cycle.

\section{REFERENCE}

1. R. T. Wood and R. B. Perez, "Modeling and Analysis of Neutron Noise from an Ex-Core Detector at a Pressurized Water Reactor," SMORN VI, A Symposium on Nuclear Reactor Surveillance and Diagnostics, Gatlinburg, Tenn., May 19-24, 1991. 
Appendix B

PHASE I MODE SHAPES 


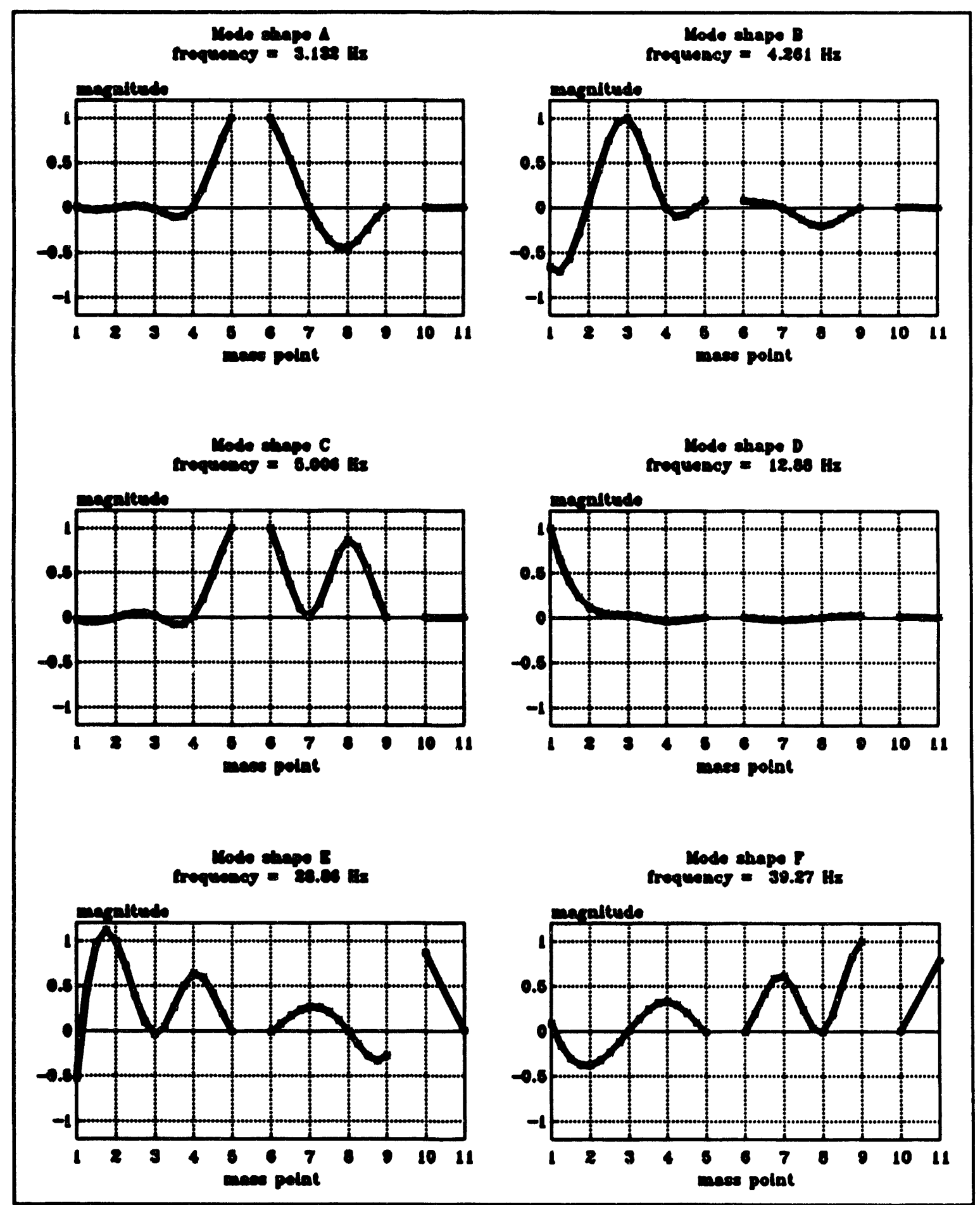

Fig. B.1. Mode shapes A through F calculated by using $K=1 \times 10^{5} \mathrm{lb} /$ in $\left(1.75 \times 10^{7} \mathrm{~N} / \mathrm{m}\right)$, $K_{m}=1 \times 10^{5} \mathrm{lb} / \mathrm{in} .\left(1.75 \times 10^{7} \mathrm{~N} / \mathrm{m}\right), E I_{s s}=1 \times 10^{6} \mathrm{lb}_{\mathrm{i}} \mathrm{in}^{2}\left(2.87 \times 10^{3} \mathrm{~N} \cdot \mathrm{m}^{2}\right)$. 


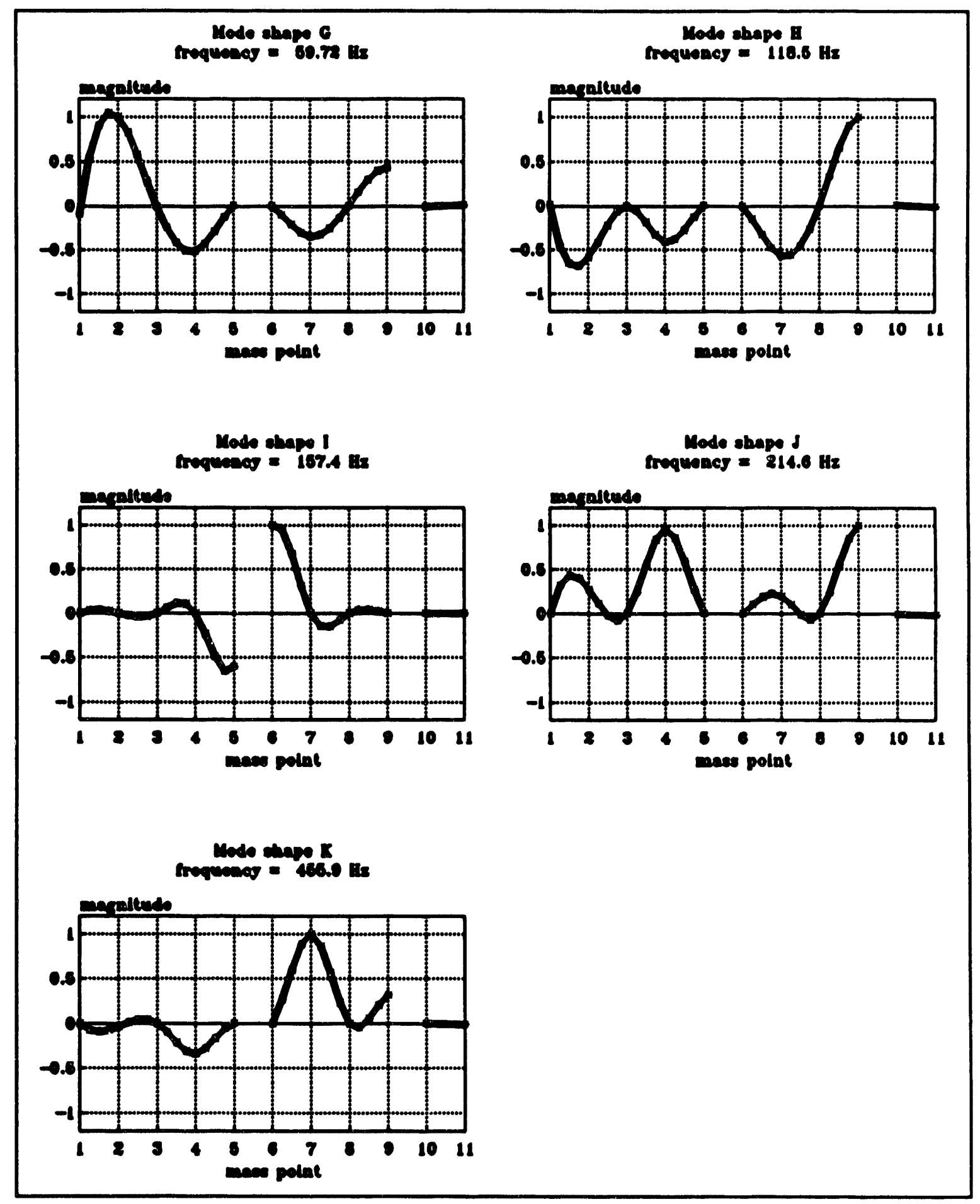

Fig. B.2 Mode shapes $G$ through $K$ calculated by using $K=1 \times 10^{5} \mathrm{lb} /$ in. $\left(1.75 \times 10^{7} \mathrm{~N} / \mathrm{m}\right)$, $K_{\mathrm{m}}=1 \times 10^{5} \mathrm{bb} / \mathrm{in} .\left(1.75 \times 10^{7} \mathrm{~N} / \mathrm{m}\right), E I_{60}=1 \times 10^{6} \mathrm{Ib}_{\mathrm{f}} \mathrm{in}^{2}\left(2.87 \times 10^{3} \mathrm{~N} \cdot \mathrm{m}^{2}\right)$. 


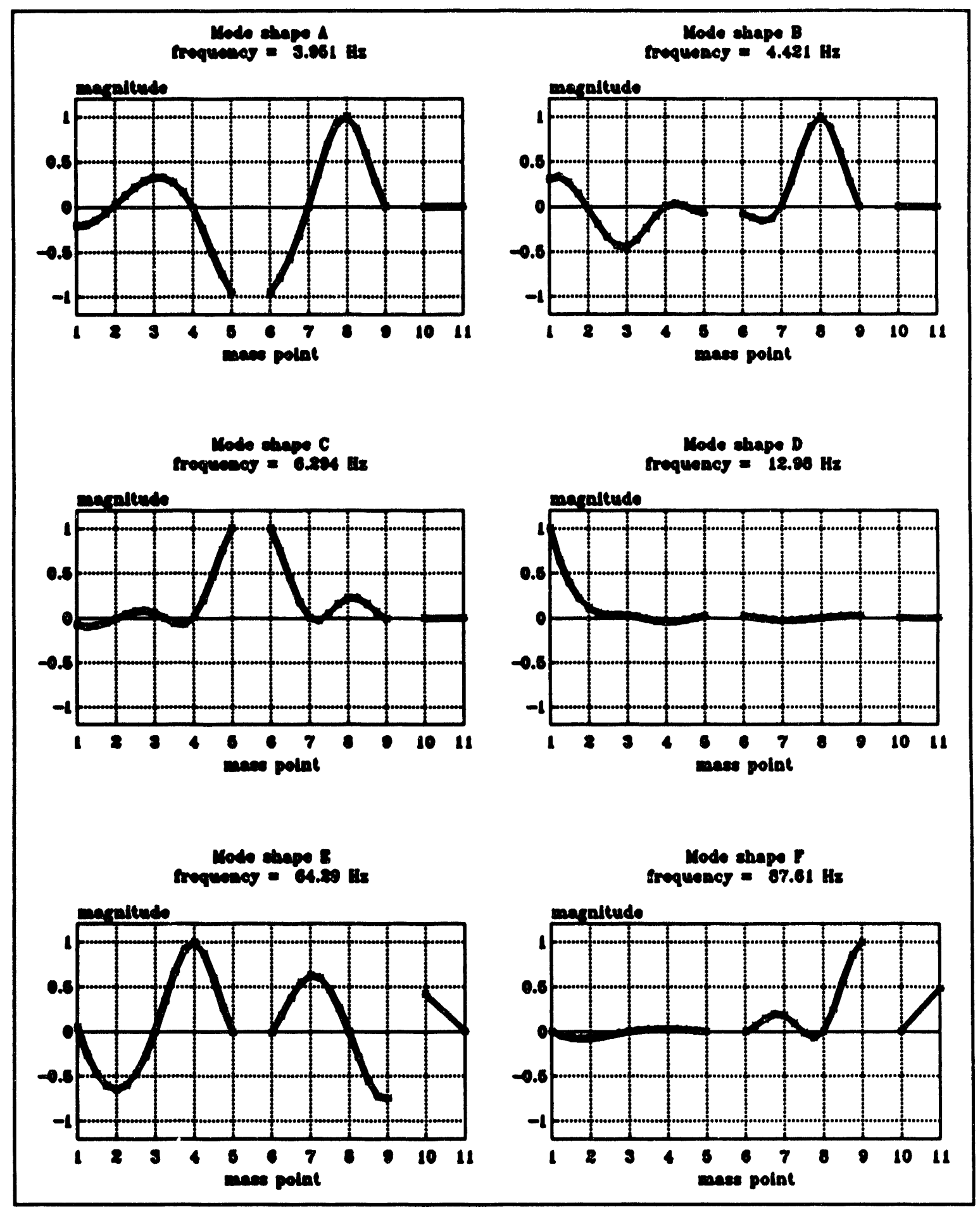

Fig B.3. Mode shapes A through F calculated by using $K_{m}=5 \times 10^{5} \mathrm{lb} / \mathrm{in} .\left(8.76 \times 10^{7} \mathrm{~N} / \mathrm{m}\right)$, $K_{\mathrm{m}}=5 \times 10^{5} \mathrm{lb} / \mathrm{in} .\left(8.76 \times 10^{7} \mathrm{~N} / \mathrm{m}\right), E I_{65}=5 \times 10^{6} \mathrm{lb}_{\mathrm{f}} \mathrm{in}^{2}\left(1.44 \times 10^{4} \mathrm{~N} \cdot \mathrm{m}^{2}\right)$. 


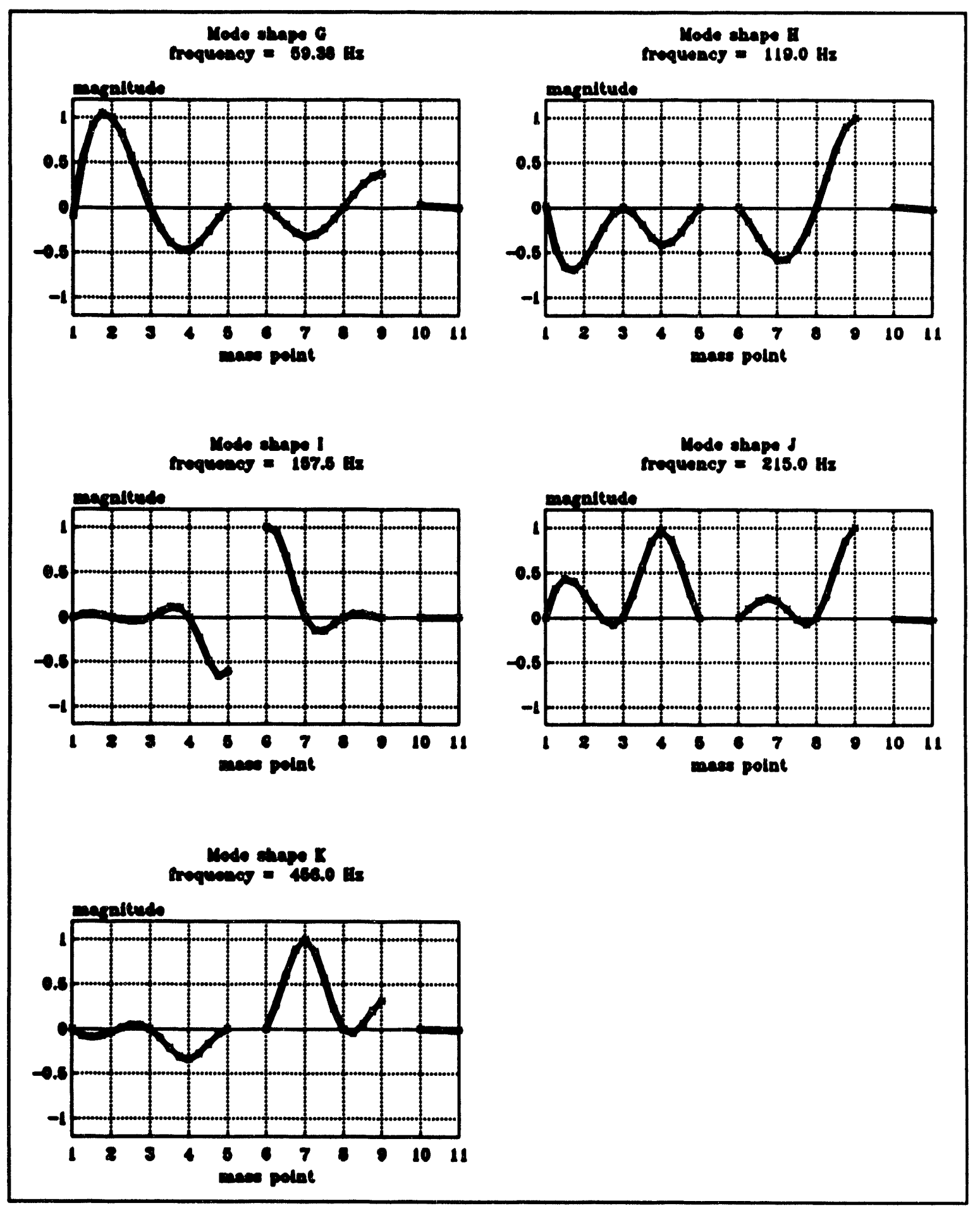

Fig. B.4. Mode shapes $G$ through $K$ calculated by using $K=5 \times 10^{5} \mathrm{lb} /$ in. $\left(8.76 \times 10^{7} \mathrm{~N} / \mathrm{m}\right)$, $K_{m}=5 \times 10^{5} \mathrm{lb} / \mathrm{in} .\left(8.76 \times 10^{7} \mathrm{~N} / \mathrm{m}\right), E I_{65}=5 \times 10^{6} \mathrm{Ib}_{\mathrm{i}}$ in $^{2}\left(1.44 \times 10^{4} \mathrm{~N} \cdot \mathrm{m}^{2}\right)$. 

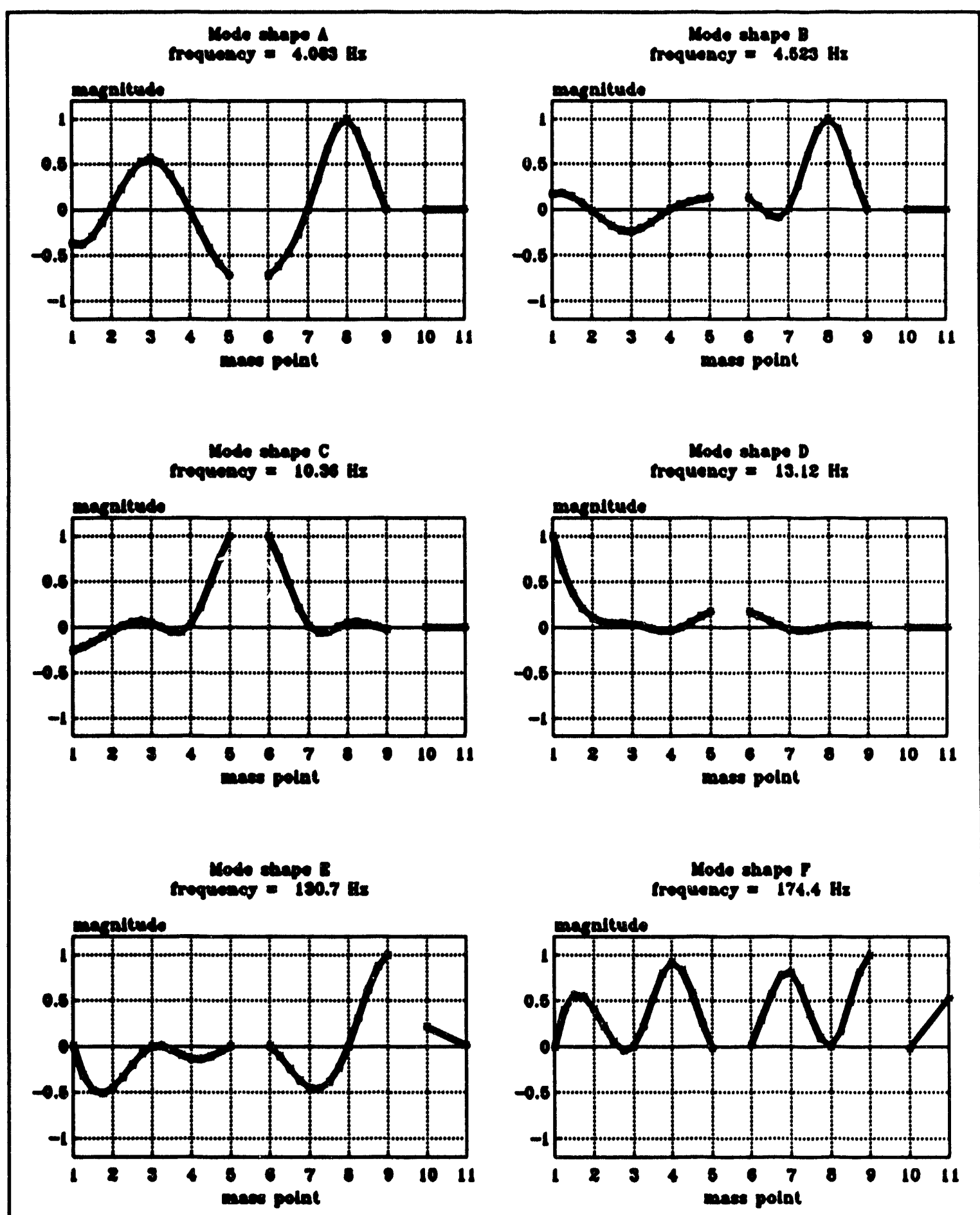

Fig. B.S. Mode shapes A through F calculated by using $K_{m}=1 \times 10^{6} \mathrm{lb} / \mathrm{in} .\left(1.75 \times 10^{8} \mathrm{~N} / \mathrm{m}\right)$, $K_{m}=1 \times 10^{6} \mathrm{lb} / \mathrm{in} .\left(1.75 \times 10^{8} \mathrm{~N} / \mathrm{m}\right), E I_{s}=1 \times 10^{7} \mathrm{lb}_{\mathrm{p}} \mathrm{in}^{2}\left(287 \times 10^{4} \mathrm{~N} \cdot \mathrm{m}^{2}\right)$. 


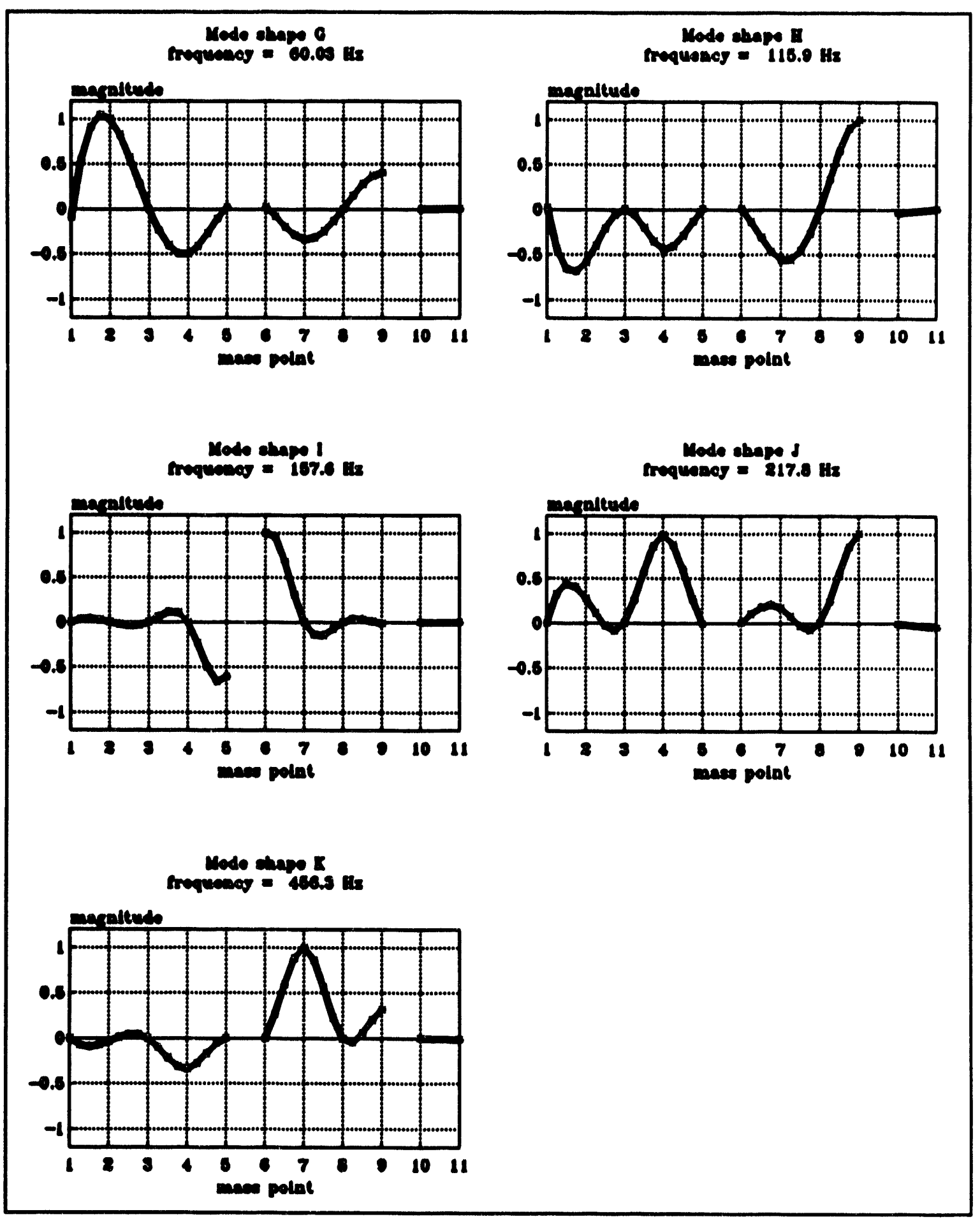

Fig. B.6. Mode shapes $G$ through $K$ calculated by using $K=1 \times 10^{6} \mathrm{lb} / \mathrm{in}$. $\left(1.75 \times 10^{8} \mathrm{~N} / \mathrm{m}\right)$, $K_{m}=1 \times 10^{6} \mathrm{lb} / \mathrm{in} .\left(1.75 \times 10^{8} \mathrm{~N} / \mathrm{m}\right), E I_{60}=1 \times 10^{7} \mathrm{lb}_{\mathrm{f}}$ in. $^{2}\left(287 \times 10^{4} \mathrm{~N} \cdot \mathrm{m}^{2}\right)$. 
Appendix C

DETAILED DRAWINGS OF THE BENCH-TOP TEST UNIT 


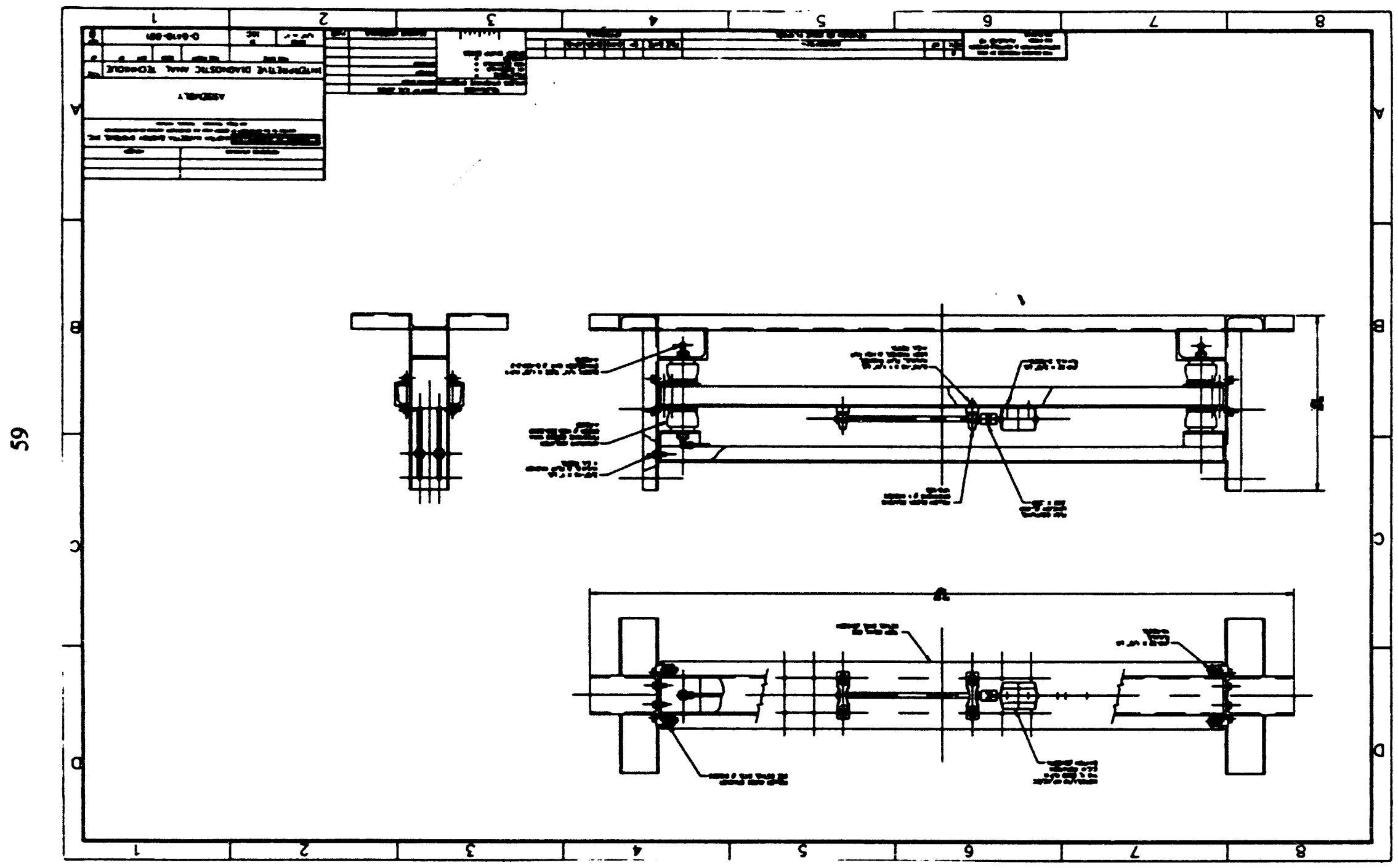




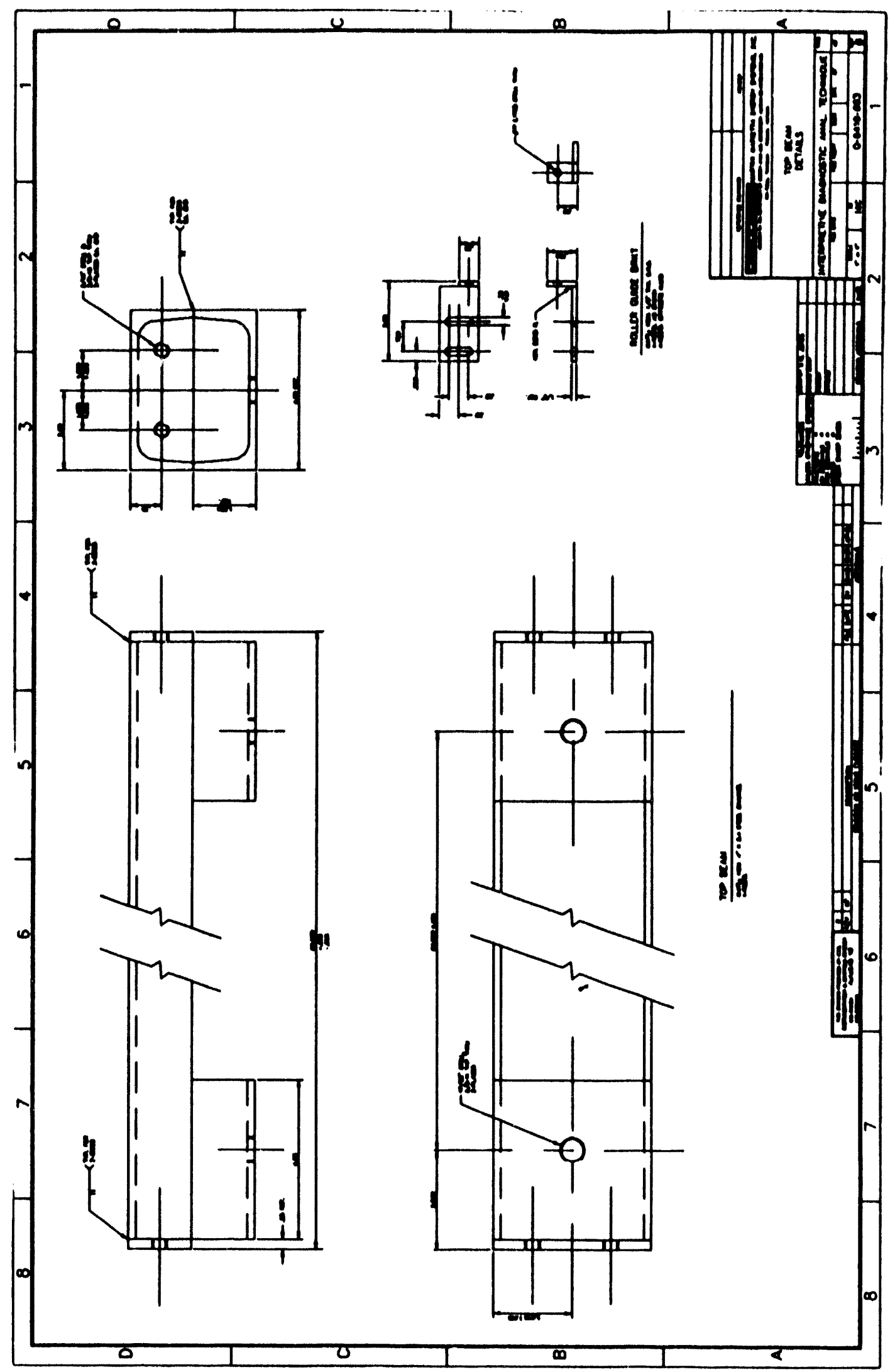




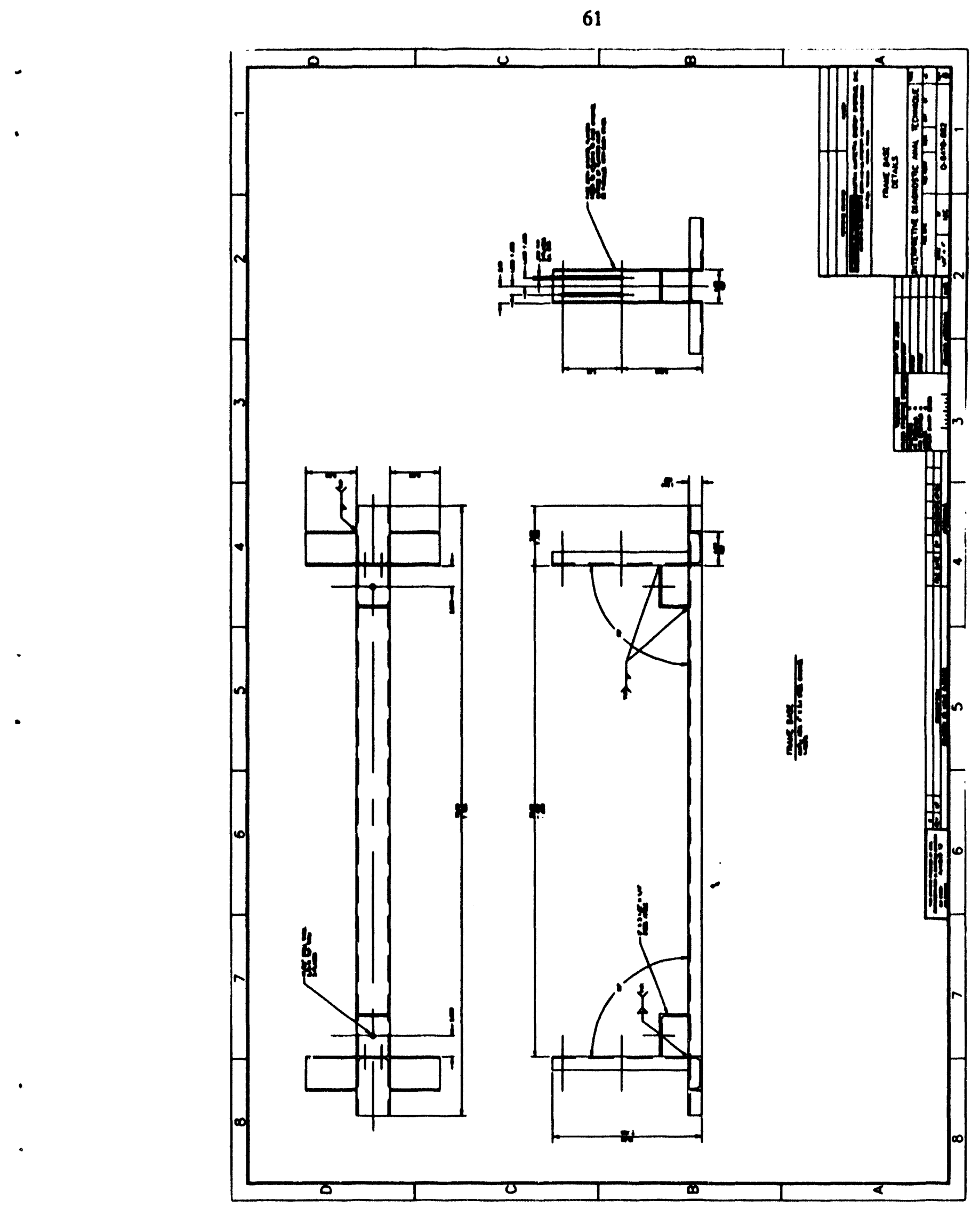


62

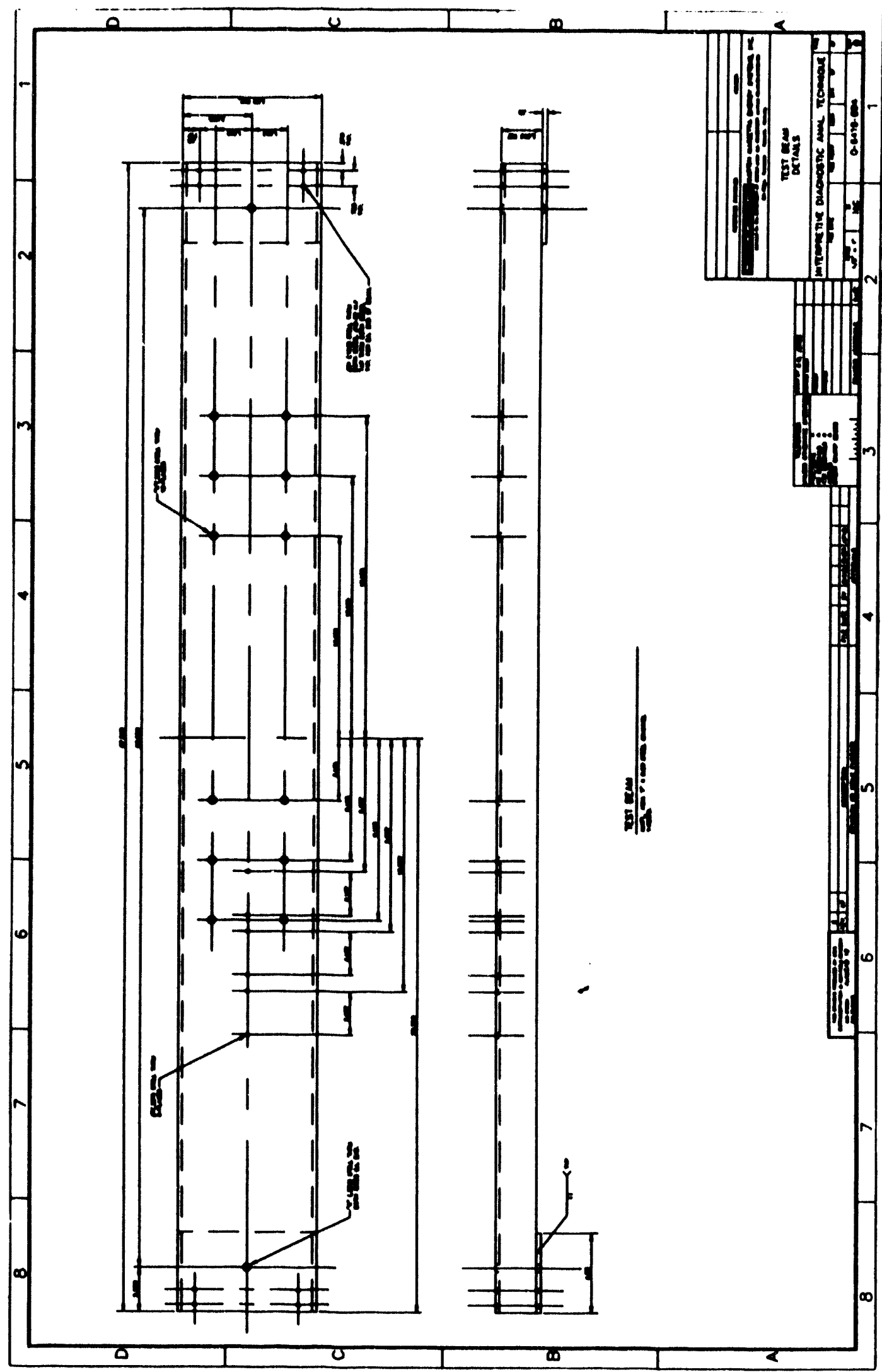


Appendix D

MEASURED RESONANCE FREQUENCIES AND MODE-SHAPE COMPONENTS FROM THE BENCH-TOP TEST UNIT 


\begin{tabular}{|c|c|c|c|c|c|}
\hline \multicolumn{2}{|c|}{$\begin{array}{c}\text { Spring rates } \\
\text { [Normalized for } 1200 \mathrm{lb} / \mathrm{in} \text {. } \\
\left.\left(2.10 \times 10^{5} \mathrm{~N} / \mathrm{m}\right)\right]\end{array}$} & \multirow{2}{*}{$\begin{array}{l}\text { Frequency } \\
(\mathrm{Hz})\end{array}$} & \multicolumn{3}{|c|}{ Mode shape component } \\
\hline$K_{\text {mom }}$ & $\mathbf{K}_{\mathrm{mp}}$ & & Left & Right & Center-of-shaft \\
\hline \multirow[t]{3}{*}{0.3421} & 0.3478 & 13.18 & 0.8412 & 0.9384 & 1.0000 \\
\hline & & 21.00 & 0.2178 & 0.8985 & 1.0000 \\
\hline & & 160.60 & 1.0000 & 0.0137 & 0.0109 \\
\hline \multirow[t]{3}{*}{0.4628} & 0.3478 & 13.67 & 0.7495 & 0.7289 & 1.0000 \\
\hline & & 21.48 & 0.1786 & 1.0000 & 0.9808 \\
\hline & & 161.10 & 1.0000 & 0.0172 & 0.0112 \\
\hline \multirow[t]{3}{*}{0.5778} & 0.3421 & 13.67 & 0.7162 & 0.5792 & 1.0000 \\
\hline & & 22.95 & 0.1223 & 1.0000 & 0.8818 \\
\hline & & 161.60 & 1.0000 & 0.0135 & 0.0139 \\
\hline \multirow[t]{3}{*}{0.6928} & 0.3133 & 13.67 & 0.6551 & 0.4666 & 1.0000 \\
\hline & & 23.44 & 0.0954 & 1.0000 & 0.7745 \\
\hline & & 161.10 & 1.0000 & 0.0148 & 0.0147 \\
\hline \multirow[t]{3}{*}{0.8078} & 0.3133 & 14.16 & 0.6173 & 0.3716 & 1.0000 \\
\hline & & 24.90 & 0.0858 & 1.0000 & 0.7138 \\
\hline & & 161.10 & 1.0000 & 0.0162 & 0.0105 \\
\hline \multirow[t]{3}{*}{0.9458} & 0.3133 & 14.16 & 0.5973 & 0.3252 & 1.0000 \\
\hline & & 27.34 & 0.1125 & 1.0000 & 0.6797 \\
\hline & & 161.10 & 1.0000 & 0.0135 & 0.0110 \\
\hline \multirow[t]{3}{*}{0.3306} & 0.4456 & 13.18 & 0.7446 & 1.0000 & 0.8009 \\
\hline & & 21.48 & 0.2567 & 0.7896 & 1.0000 \\
\hline & & 160.60 & 1.0000 & 0.0157 & 0.0116 \\
\hline
\end{tabular}




\begin{tabular}{|c|c|c|c|c|c|}
\hline \multicolumn{2}{|c|}{$\begin{array}{c}\text { Spring rates } \\
\text { [Normalized for } 1200 \mathrm{lb} / \mathrm{in} \text {. } \\
\left.\left(2.10 \times 10^{5} \mathrm{~N} / \mathrm{m}\right)\right]\end{array}$} & \multirow{2}{*}{$\begin{array}{c}\text { Frequency } \\
(\mathrm{Hz})\end{array}$} & \multicolumn{3}{|c|}{ Mode shape component } \\
\hline$K_{\text {mo }}$ & $\mathbf{K}_{\mathrm{mp}}$ & & Left & Right & Center-of-shaft \\
\hline \multirow[t]{3}{*}{0.4456} & 0.4398 & 14.16 & 0.8202 & 0.8722 & 1.0000 \\
\hline & & 21.97 & 0.2138 & 0.9207 & 1.0000 \\
\hline & & 160.60 & 1.0000 & 0.0130 & 0.0096 \\
\hline \multirow[t]{3}{*}{0.5721} & 0.4571 & 14.65 & 0.7295 & 0.7039 & 1.0000 \\
\hline & & 23.44 & 0.1607 & 1.0000 & 0.9603 \\
\hline & & 160.60 & 1.0000 & 0.0181 & 0.0117 \\
\hline \multirow[t]{3}{*}{0.6813} & 0.4571 & 14.89 & 0.7162 & 0.5725 & 1.0000 \\
\hline & & 24.41 & 0.1305 & 1.0000 & 0.8785 \\
\hline & & 160.60 & 1.0000 & 0.0157 & 0.0094 \\
\hline \multirow[t]{3}{*}{0.7791} & 0.4283 & 15.14 & 0.6792 & 0.5208 & 1.0000 \\
\hline & & 24.41 & 0.1253 & 1.0000 & 0.8494 \\
\hline & & 160.20 & 1.0000 & 0.0163 & 0.0141 \\
\hline \multirow[t]{3}{*}{0.9228} & 0.4456 & 15.14 & 0.6737 & 0.4725 & 1.0000 \\
\hline & & 26.86 & 0.0962 & 1.0000 & 0.7588 \\
\hline & & 160.60 & 1.0000 & 0.0167 & 0.0097 \\
\hline \multirow[t]{3}{*}{0.3306} & 0.5606 & 13.67 & 0.6922 & 1.0000 & 0.6747 \\
\hline & & 21.97 & 0.3009 & 0.7312 & 1.0000 \\
\hline & & 160.60 & 1.0000 & 0.0124 & 0.0121 \\
\hline \multirow[t]{3}{*}{0.4571} & 0.5606 & 14.65 & 0.8602 & 0.9838 & 1.0000 \\
\hline & & 23.44 & 0.2277 & 0.8832 & 1.0000 \\
\hline & & 160.60 & 1.0000 & 0.0127 & 0.0108 \\
\hline
\end{tabular}




\begin{tabular}{|c|c|c|c|c|c|}
\hline \multicolumn{2}{|c|}{$\begin{array}{c}\text { Spring rates } \\
\text { [Normalized for } 1200 \mathrm{lb} / \mathrm{in} . \\
\left.\left(2.10 \times 10^{5} \mathrm{~N} / \mathrm{m}\right)\right]\end{array}$} & \multirow{2}{*}{$\begin{array}{l}\text { Frequency } \\
(\mathrm{Hz})\end{array}$} & \multicolumn{3}{|c|}{ Mode shape component } \\
\hline $\mathbf{K}_{\max }$ & $\mathbf{K}_{\mathbf{m p}}$ & & Left & Right & Center-of-shaft \\
\hline \multirow[t]{3}{*}{0.5433} & 0.5663 & 15.14 & 0.8865 & 0.9971 & 1.0000 \\
\hline & & 23.44 & 0.2473 & 0.8699 & 1.0000 \\
\hline & & 161.10 & 1.0000 & 0.0153 & 0.0130 \\
\hline \multirow[t]{3}{*}{0.6756} & 0.5606 & 15.62 & 0.7935 & 0.7748 & 1.0000 \\
\hline & & 24.41 & 0.2185 & 0.9904 & 1.0000 \\
\hline & & 161.10 & 1.0000 & 0.0138 & 0.0110 \\
\hline \multirow[t]{3}{*}{0.8021} & 0.5606 & 16.11 & 0.7557 & 0.6273 & 1.0000 \\
\hline & & 26.12 & 0.1695 & 1.0000 & 0.8786 \\
\hline & & 161.10 & 1.0000 & 0.0151 & 0.0110 \\
\hline \multirow[t]{3}{*}{0.8998} & 0.5548 & 16.11 & 0.7090 & 0.5543 & 1.0000 \\
\hline & & 27.83 & 0.1162 & 1.0000 & 0.8121 \\
\hline & & 161.10 & 1.0000 & 0.0152 & 0.0119 \\
\hline \multirow[t]{3}{*}{0.3363} & 0.6756 & 14.16 & 0.6301 & 1.0000 & 0.5895 \\
\hline & & 22.95 & 0.3097 & 0.6769 & 1.0000 \\
\hline & & 160.60 & 1.0000 & 0.0149 & 0.0126 \\
\hline \multirow[t]{3}{*}{0.4513} & 0.6698 & 15.14 & 0.8016 & 1.0000 & 0.8414 \\
\hline & & 23.44 & 0.2729 & 0.8114 & 1.0000 \\
\hline & & 160.60 & 1.0000 & 0.0152 & 0.0099 \\
\hline \multirow[t]{3}{*}{0.5663} & 0.6698 & 15.62 & 0.8867 & 0.9685 & 1.0000 \\
\hline & & 24.90 & 0.2496 & 0.9153 & 1.0000 \\
\hline & & 160.60 & 1.0000 & 0.0138 & 0.0085 \\
\hline
\end{tabular}


68

\begin{tabular}{|c|c|c|c|c|c|}
\hline \multicolumn{2}{|c|}{$\begin{array}{c}\text { Spring rates } \\
\text { [Normalized for } 1200 \mathrm{lb} / \text { in } \\
\left.\left(2.10 \times 10^{5} \mathrm{~N} / \mathrm{m}\right)\right]\end{array}$} & \multirow{2}{*}{$\begin{array}{l}\text { Frequency } \\
\qquad(\mathrm{Hz})\end{array}$} & \multicolumn{3}{|c|}{ Mode shape component } \\
\hline $\mathbf{K}_{\operatorname{mox}}$ & $\mathbf{K}_{\mathrm{mp}}$ & & Left & Right & Center-of-shaft \\
\hline \multirow[t]{3}{*}{0.6871} & 0.6756 & 16.11 & 0.8151 & 0.8322 & 1.0000 \\
\hline & & 25.88 & 0.2108 & 1.0000 & 0.9882 \\
\hline & & 161.10 & 1.0000 & 0.0158 & 0.0112 \\
\hline \multirow[t]{3}{*}{0.7963} & 0.6756 & 16.60 & 0.8107 & 0.6958 & 1.0000 \\
\hline & & 27.34 & 0.1575 & 1.0000 & 0.8846 \\
\hline & & 160.60 & 1.0000 & 0.0136 & 0.0083 \\
\hline \multirow[t]{3}{*}{0.9228} & 0.6756 & 17.09 & 0.7771 & 0.6287 & 1.0000 \\
\hline & & 28.32 & 0.1230 & 1.0000 & 0.8355 \\
\hline & & 160.20 & 1.0000 & 0.0154 & 0.0104 \\
\hline \multirow[t]{3}{*}{0.3248} & 0.7733 & 14.16 & 0.5672 & 1.0000 & 0.5094 \\
\hline & & 23.44 & 0.3400 & 0.6447 & 1.0000 \\
\hline & & 162.60 & 1.0000 & 0.0128 & 0.0123 \\
\hline \multirow[t]{3}{*}{0.4513} & 0.7906 & 15.14 & 0.7171 & 1.0000 & 0.6705 \\
\hline & & 25.63 & 0.3688 & 0.7804 & 1.0000 \\
\hline & & 161.60 & 1.0000 & 0.0127 & 0.0105 \\
\hline \multirow[t]{3}{*}{0.5663} & 0.7848 & 16.11 & 0.8385 & 1.0000 & 0.8488 \\
\hline & & 26.37 & 0.3200 & 0.9036 & 1.0000 \\
\hline & & 161.10 & 1.0000 & 0.0148 & 0.0088 \\
\hline \multirow[t]{3}{*}{0.6928} & 0.7906 & 16.60 & 0.8929 & 1.0000 & 0.9763 \\
\hline & & 27.34 & 0.2692 & 0.9893 & 1.0000 \\
\hline & & 161.10 & 1.0000 & 0.0114 & 0.0100 \\
\hline
\end{tabular}


69

\begin{tabular}{|c|c|c|c|c|c|}
\hline \multicolumn{2}{|c|}{$\begin{array}{c}\text { Spring rates } \\
\text { [Normalized for } 1200 \mathrm{lb} / \text { in. } \\
\left.\left(2.10 \times 10^{5} \mathrm{~N} / \mathrm{m}\right)\right]\end{array}$} & \multirow{2}{*}{$\begin{array}{l}\text { Frequency } \\
(\mathrm{Hz})\end{array}$} & \multicolumn{3}{|c|}{ Mode shape component } \\
\hline$K_{\text {mod }}$ & $K_{\text {mp }}$ & & Left & Right & Center-of-shaft \\
\hline \multirow[t]{3}{*}{0.8078} & 0.7906 & 17.09 & 0.9204 & 0.8769 & 1.0000 \\
\hline & & 28.07 & 0.2034 & 1.0000 & 0.9030 \\
\hline & & 161.10 & 1.0000 & 0.0133 & 0.0108 \\
\hline \multirow[t]{3}{*}{0.9458} & 0.7906 & 17.58 & 0.8184 & 0.7387 & 1.0000 \\
\hline & & 28.81 & 0.1671 & 1.0000 & 0.8662 \\
\hline & & 161.10 & 1.0000 & 0.0155 & 0.0121 \\
\hline \multirow[t]{3}{*}{0.3306} & 0.8998 & 14.65 & 0.5926 & 1.0000 & 0.4767 \\
\hline & & 25.39 & 0.4139 & 0.7043 & 1.0000 \\
\hline & & 160.60 & 1.0000 & 0.0154 & 0.0103 \\
\hline \multirow[t]{3}{*}{0.4513} & 0.9056 & 15.62 & 0.6396 & 1.0000 & 0.5803 \\
\hline & & 26.37 & 0.3812 & 0.7852 & 1.0000 \\
\hline & & 160.60 & 1.0000 & 0.0156 & 0.0119 \\
\hline \multirow[t]{3}{*}{0.5663} & 0.9056 & 16.60 & 0.7901 & 1.0000 & 0.7037 \\
\hline & & 26.86 & 0.3531 & 0.8955 & 1.0000 \\
\hline & & 161.10 & 1.0000 & 0.0149 & 0.0103 \\
\hline \multirow[t]{3}{*}{0.6928} & 0.9056 & 17.09 & 0.9126 & 1.0000 & 0.9335 \\
\hline & & 27.83 & 0.2817 & 1.0000 & 0.9851 \\
\hline & & 160.60 & 1.0000 & 0.0150 & 0.0121 \\
\hline \multirow[t]{3}{*}{0.8078} & 0.9056 & 17.58 & 0.9362 & 0.9588 & 1.0000 \\
\hline & & 28.32 & 0.2554 & 1.0000 & 0.9595 \\
\hline & & 160.60 & 1.0000 & 0.0149 & 0.0114 \\
\hline
\end{tabular}




\begin{tabular}{|c|c|c|c|c|c|}
\hline \multicolumn{2}{|c|}{$\begin{array}{l}\text { Spring rates } \\
\text { [Normalized for } 1200 \mathrm{lb} / \mathrm{in} \text {. } \\
\left.\left(2.10 \times 10^{5} \mathrm{~N} / \mathrm{m}\right)\right]\end{array}$} & \multirow{2}{*}{$\begin{array}{l}\text { Frequency } \\
(\mathrm{Hz})\end{array}$} & \multicolumn{3}{|c|}{ Mode shape component } \\
\hline $\mathbf{K}_{\operatorname{mon}}$ & $\mathbf{K}_{\mathbf{m p}}$ & & Left & Right & Center-of-shaft \\
\hline \multirow[t]{3}{*}{0.9056} & 0.9171 & 17.58 & 0.9416 & 0.9494 & 1.0000 \\
\hline & & 28.32 & 0.2217 & 1.0000 & 0.9293 \\
\hline & & 160.60 & 1.0000 & 0.0136 & 0.0099 \\
\hline
\end{tabular}




\section{INTERNAL DISTRIBUTION}

1-10. B. Damiano

11-15. E. D. Blakeman

16-20. L. D. Phillips

21. Y. Bartel

22. H. R. Brashear

23. N. E. Clapp, Jr.

24. D. N. Fry

25. R. A. Hess

26. R. A. Kisner

27. K. Korsah

28. J. Lin

29. D. W. McDonald

30. S. R. McNeany

31. D. R. Miller

32. G. N. Miller
33. J. A. Mullens

34-39. J. R. Palmer

40. M. E. Reeves

41. J. O. Stiegler

42. R. E. Uhrig

43. J. D. White

44. R. T. Wood

45. D. F. Craig

46-47. Central Research Library

48. Y-12 Technical Reference Section

49-50. Laboratory Records

51. Laboratory Records-Record Copy

52. ORNL Patent Section

53. I\&C Division Publications Office

\section{EXTERNAL DISTRIBUTION}

54. Assistant Manager for Energy Research and Development, DOE-ORO, P.O. Box 2001, Oak Ridge, TN 37831-8600

55. B. Chexal, Electric Power Research Institute, 3412 Hillview Avenue, Palo Alto, CA 94303

56. V. Radeka, Brookhaven National Laboratory, Instrumentation Division, 535-B, Upton, NY 11973

57. M. M. Sevik, Carderock Division, Naval Surface Warfare Center, Code 1900, Bethesda, MD 20084-5000

58. R. M. Taylor, Leeds and Northup, Sunneytown Pike, North Wales, PA 19454

59-60. Office of Scientific and Technical Information, U.S. Department of Energy, P.O. Box 62, Oak Ridge, TN 37831 

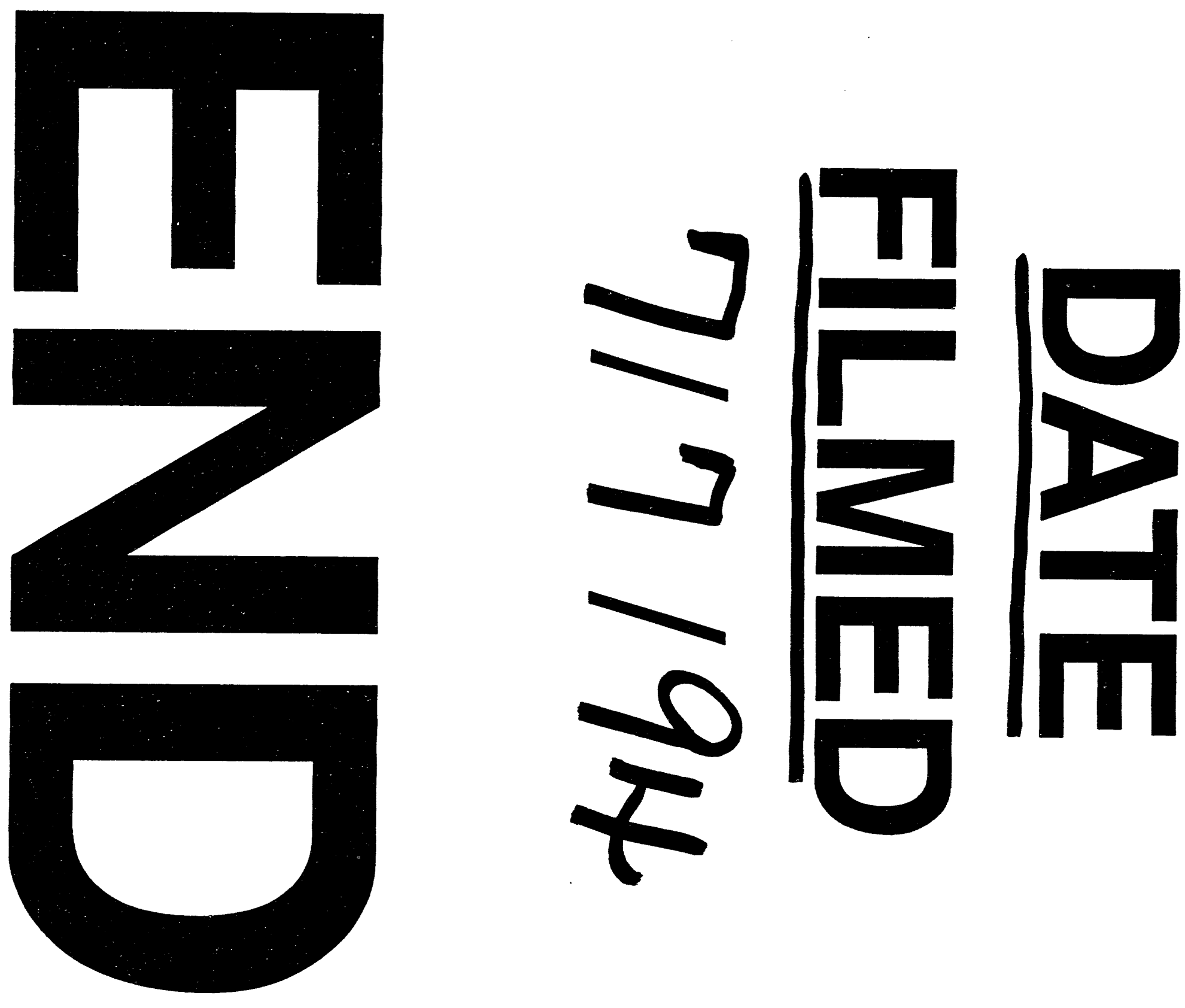
